Copyright ACM, 2015. This is the author's version of the work. It is posted here by permission of ACM for your personal use. Not for redistribution.

The definitive version was published in ACM Transactions on Computational Logic (TOCL), vol 16, iss 4, November 2015, http.// doi.org/10.1145/2786759.

\title{
DL-Lite Ontology Revision Based on An Alternative Semantic Characterisation
}

\author{
ZHE WANG, KEWEN WANG, and RODNEY TOPOR, Griffith University
}

\begin{abstract}
Ontology engineering and maintenance require (semi-)automated ontology change operations. Intensive research has been conducted on TBox and ABox changes in description logics (DLs), and various change operators have been proposed in the literature. Existing operators largely fall into two categories: syntax-based and model-based. While each approach has its advantages and disadvantages, an important topic that has been rarely explored is how to achieve a balance between syntax-based and model-based approaches. Also, most existing operators are specially designed for either TBox change or ABox change, and cannot handle the general ontology revision task-Given a DL knowledge base (KB, a pair of a TBox and an ABox), how to revise it by a set of TBox and ABox axioms (i.e., a new DL KB). In this paper, we introduce an alternative structure for DL-Lite, called a featured interpretation, and show that featured models provide a finite and tight characterization to the classical semantics of DL-Lite. A key issue for defining a change operator is the so-called expressibility, that is, whether a set of models (or featured models here) is axiomatizable in DLs. It is indeed much easier to obtain expressibility results for featured models than for classical DL models. As a result, the new semantics determined by featured models provides a method for defining and studying various changes of DL-Lite KBs that involve both TBoxes and ABoxes. To demonstrate the usefulness of the new semantic characterization in ontology change, we define two revision operators for DL-Lite KBs using featured models and study their properties. In particular, we show that our two operators both satisfy AGM postulates. We show that the complexity of our revisions is $\Pi_{2}^{P}$-complete, that is, on the same level as major revision operators in propositional logic, which further justifies the feasibility of our revision approach for DL-Lite. Also, we develop algorithms for these DL-Lite revisions.
\end{abstract}

Categories and Subject Descriptors: I.2.3 [Deduction and Theorem Proving]: Nonmonotonic reasoning and belief revision; I.2.4 [Knowledge Representation Formalisms and Methods]: Representation languages; F.4.1 [Mathematical Logic]: Computational logic

General Terms: Theory

Additional Key Words and Phrases: Description logics, DL-Lite, ontology change, revision

ACM Reference Format:

Zhe Wang, Kewen Wang, and Rodney Topor, 2014. DL-Lite ontology revision based on an alternative semantic characterisation. ACM Trans. Comput. Logic V, N, Article A (January YYYY), 45 pages.

DOI : http://dx.doi.org/10.1145/0000000.0000000

\section{INTRODUCTION}

Ontology has recently been used in a wide range of practical domains such as e-Science, eCommerce, medical informatics, bio-informatics, and the Semantic Web [Staab and Studer 2009]. An ontology is a formal model of some domain knowledge of the world, and it provides a shared vocabulary relevant to the domain. It also specifies the formalization of the domain knowledge as well as the meaning (semantics) of the formalization. The Web Ontology Language (OWL), with its latest version, OWL 21 is accepted as the World Wide Web Consortium (W3C) recommendation for

${ }^{1}$ http://www.w3.org/TR/owl2-overview/

Author's addresses: Z. Wang, K. Wang, and R. Topor, School of ICT, Griffith University.

This paper is a significant extension of [Wang et al. 2010a. Wang et al. 2010b] ([Wang et al. 2010b] is an extended abstract of [Wang et al. 2010a]).

Permission to make digital or hard copies of part or all of this work for personal or classroom use is granted without fee provided that copies are not made or distributed for profit or commercial advantage and that copies show this notice on the first page or initial screen of a display along with the full citation. Copyrights for components of this work owned by others than ACM must be honored. Abstracting with credit is permitted. To copy otherwise, to republish, to post on servers, to redistribute to lists, or to use any component of this work in other works requires prior specific permission and/or a fee. Permissions may be requested from Publications Dept., ACM, Inc., 2 Penn Plaza, Suite 701, New York, NY 10121-0701 USA, fax +1 (212) 869-0481, or permissions@acm.org.

(C) YYYY ACM 1529-3785/YYYY/01-ARTA $\$ 15.00$

DOI : http://dx.doi.org/10.1145/0000000.0000000 
ontology languages. OWL and OWL 2 are based on description logics (DLs) [Baader et al. 2003]. In particular, the logical foundations of OWL $2 \mathrm{QL}$ are based on a well known family of lightweight DLs, DL-Lite [Calvanese et al. 2007; Artale et al. 2009]. In DLs, an ontology is often expressed as a knowledge base $(K B)$, which consists of both terminological knowledge (or schema information) in the TBOx and assertional knowledge (or data information) in the ABox. We will alternatively use the terms 'ontology' and 'KB'.

As with all formal knowledge structures, ontologies are not static, but may evolve over time. Indeed, ontology engineering is described as a life-cycle [Pérez et al. 2003], which is based on evolving prototypes and specific techniques peculiar to each ontology engineering activity. An important and challenging problem is thus how to effectively and efficiently modify ontologies. A typical scenario is machine-supported ontology design and maintenance through a learning process - this may involve sophisticated change operations, in particular when a piece of newly learned knowledge contradicts the initial knowledge, the ontology needs to be revised or a recommendation for such a revision should be generated for the users. For example, suppose we need to build an ontology about the staff and the students in a community college. Initially, we may have defined two disjoint classes Staff and Student among some other classes. Assume that the ontology contains some schema-level information, such as staff have staff IDs and students do not. Also, the ontology contains some data about the staff and the students in the college, e.g., Mary is a staff member, John and Mike are students. Later, however, we learn that the college allows some of its staff members to enrol as $\mathrm{PhD}$ students. Also, John has graduated from his PhD program and is now a staff member in the college. Then, the new knowledge needs to be incorporated and the initial ontology (both the TBox and the ABox) needs to be revised so that the incorporation is consistent.

Limited support on ontology change is provided by existing ontology editing tools. In particular, Protég $\AA^{2}$ a well known ontology editor and knowledge acquisition system, allows the users to incorporate one ontology into another; however, the interactions between the ontologies are restricted. For instance, classes from distinct sources with the same name are not identified as the same class by default. When incorporating two ontologies in Protégé, classes with the same name co-exist in the resulting ontology. For instance, suppose we have two ontologies both with a class called Student. When merging the two ontologies, two classes both named Student will occur in the result. The two classes can only be distinguished when we refer to their respective URIs inherited from their source ontologies. Suppose these two classes are unified (for instance via mapping), another issue still remains - the two source ontologies may contain inconsistent knowledge about this unified class. Yet insufficient support is provided by Protégé to resolve such inconsistency; while it can detect such inconsistency [Horridge et al. 2009], it relies on human assistance to understand and resolve the inconsistency.

Recently, significant effort has been made to define rational operators for DL ontology change and a number of proposals have been studied under various names, such as revision [Qi and Yang 2008; Nikitina et al. 2012], contraction [Cuenca Grau et al. 2012], update [Kharlamov et al. 2013], forgetting [Wang et al. 2010c; Lutz and Wolter 2011], and module extraction [Cuenca Grau et al. 2008; Kontchakov et al. 2010|. The list is by no means comprehensive, the readers should refer to [Flouris et al. 2008] for a survey on some other related works. While some of the existing approaches are promising, the following two problems are still open.

(1). How to achieve a balance between syntax-based and model-based approaches which has advantages of each approach? Current approaches to ontology change can be classified (roughly) into two categories: syntax-based approaches [Haase and Stojanovic 2005; Qi et al. 2008; Ribeiro and Wassermann 2009] and model-based approaches [Giacomo et al. 2009, Qi and Du 2009; Zheleznyakov et al. 2010; Lenzerini and Savo 2011; Liu et al. 2011; Kharlamov et al. 2013; Qi et al. 2015|. It is well known that these two types of proposals for ontology change have their advantages and disadvantages. A syntax-based approach is usually based on certain syntactic

\footnotetext{
${ }^{2} \mathrm{http}: / /$ protege.stanford.edu/
} 
structures instead of standard DL models. Such approaches are relatively easy to implement, but they lack of suitable semantic justification in general, that is, it is usually unclear how close (semantically) the changed KB is to the initial one (except for some special cases). Model-based approaches are defined in terms of DL models and thus provide a natural semantic justification of minimal change. However, it is challenging to develop algorithms for model-based KB changes except for some special cases (e.g., TBox or ABox changes, a detailed discussion is left to Section 6). In our view, one of the major difficulties is to work with (essentially first order) DL models, for the following reasons: (i) DL models have complex and possibly infinite structures; (ii) a DL KB may have infinitely many models, making it hard (if not impossible) to compute the result directly via models; and (iii) given a collection of DL models, there may not exist a single KB whose models are exactly those in the set.

(2). How to develop a framework that allows us to change both the TBox and the ABox in a simple, uniform and well-defined manner? As we have seen from the above community college ontology example, it is necessary to change both the TBox and the ABox of a DL KB, while we agree that there are applications for which only TBoxes or only ABoxes need to change. In Section 4.4, we will describe a case study of ontology revision on the NCI Thesaurus [Hartel et al. 2005] to demonstrate that both the ABox and the TBox need to be revised. A limitation of many existing approaches is that they can either modify only ABoxes with fixed TBoxes [Giacomo et al. 2009, Lenzerini and Savo 2011, Liu et al. 2011, Kharlamov et al. 2013, Qi et al. 2015|, or change only TBoxes [Qi and Du 2009, Zheleznyakov et al. 2010; Zhuang et al. 2014; Wang et al. 2015]. Revision by a distinct type of axioms, e.g., revision of TBoxes by ABoxes, was rarely explored with the exception of [Wang et al. 2015], where an approach is proposed to revise a TBox by some contradicting ABox. This is the case for example, suppose the new data shows that $\mathrm{PhD}$ students like John get a staff ID which was not permitted before, then the TBox constraint needs to be revised. Yet the other direction is not covered by the existing approaches. That is, in a knowledge intensive application where the data is error-prone and frequently updated, when new integrity constraints is applied the out-of-date initial ABox needs to be revised by the new TBox knowledge. For instance, suppose the new TBox constraint says each person can have only one ID, then the incorrect assertion that John has two distinct IDs must be revised.

In order to resolve the above two challenges, in this paper we first introduce a new type of syntactic structures called featured interpretations for DL-Lite, which provides a suitable finite characterisation for the semantics of DL-Lite KBs, and then we apply this new semantic characterisation in defining revision operators for DL-Lite KBs and developing algorithms for the revision operators.

We choose DL-Lite [Calvanese et al. 2007; Artale et al. 2009] for several reasons. DL-Lite was developed as a family of lightweight DLs that exhibit nice computational properties. DL-Lite is the logical foundation of OWL 2 QL, one of the three profiles of W3C standard OWL 2. Moreover, while it is essentially a fragment of first order logic, DL-Lite is relatively close to propositional logic in the sense that quite some important properties of propositional logic can be adapted/extended to DL-Lite. In particular, we use DL-Lite ${ }_{\text {bool }}^{\mathcal{N}}$ [Artale et al. 2009] as a syntactic basis for our discussion as it generalizes the main DL-Lite dialects such as DL-Lite core $_{\text {and DL-Lite }}$, and is expressive enough to allow all boolean operators. Also, the proposed approach can be conveniently adapted to other DL-Lite dialects like DL-Lite ${ }_{\mathcal{R}}$.

The notion of types [Kontchakov et al. 2010] — syntactic abstractions of domain elementsprovides a finite characterisation for the semantics of DL-Lite TBoxes. But single types cannot be directly used as models of DL-Lite KBs that have both TBoxes and ABoxes, as we will see in Example 3.9 As a result, we introduce a new type-based structure, called a featured interpretation, for DL-Lite, and show that the notion of featured interpretations provides a nice characterisation for the semantics of DL-Lite KBs, in particular, a DL-Lite KB is consistent (in terms of DL models) if and only if it is consistent in terms of featured models (and it is well known that most DL-Lite reasoning tasks can be reduced to KB consistency check [Artale et al. 2009]). 
The new semantic characterisation can be used for resolving conflicts, handling revision, update, forgetting, and merging. As an application of featured models, we present a thorough account of how featured models are used in DL-Lite ontology revision. In particular, we propose two revision operators by employing techniques of belief revision in propositional logic, show that our operators satisfy AGM postulates for revision, and develop algorithms for our revision operators. As a case study, we present an example of using our operators for medical ontology revision, based on a slightly modified version of the NCI Thesaurus. We also show that the computational complexity of our revision operators is on the same level as belief revision for propositional logic. Specifically, the major contributions of this work are summarized as follows.

- Featured interpretations and the corresponding semantics: Featured interpretations are syntaxbased and finite, in the sense that each DL-Lite KB has a finite set of featured interpretations that satisfy it (called featured models) and each featured interpretation is finite. The semantics determined by featured models provides a tight approximation to the classical semantics of DLLite KBs. As a result, the featured-model semantics paves the way for a good balance between syntax-based and model-based approaches to DL-Lite ontology change.

- Conditions for the expressibility of featured interpretations: The expressibility of featured interpretations concerns whether there exists a DL-Lite KB whose featured models are exactly a given set of featured interpretations. In general, a set of featured interpretations may not be expressible as a single DL-Lite KB. To resolve this problem, we first study the conditions for a set of featured interpretations to be expressible in DL-Lite. Moreover, when a set of featured interpretations is inexpressible, we show how to construct the closest DL-Lite KB corresponding to the given set of featured interpretations, called the maximal approximation.

- Two Ontology Revision Operators: By introducing two notions of distance between featured models, we define two different revision operators for DL-Lite KBs. Our revision operators balance between syntax-based and model-based revision approaches in the sense that we adapt techniques from propositional model-based revision but use featured models instead of DL models. We show that both operators possess desirable logical properties and in particular, they satisfy related AGM postulates. We still face the inexpressibility issue- the result of revision can be a disjunctive KB. Thus, we study approximation of our DL-Lite revisions. To demonstrate the usefulness of these ontology revision operators (even under approximation), we also present a case study of ontology revision based on the NCI Thesaurus.

-Algorithms and Complexity: We show that the complexity of ontology revision based on featured models is on the second level of polynomial hierarchy. This result is non-trivial and encouraging, as the complexity coincides with that of belief revision for propositional logic. We develop algorithms for computing the maximal approximation for DL-Lite ontology revision. The algorithms are sound and complete with respect to our two operators. These results are significant, considering the generality of the revision task considered in this paper and the difficulty of developing an algorithm for a revision operator defined in terms of DL models.

The rest of the paper is arranged as follows. Some basics of DL-Lite ${ }_{\text {bool }}^{\mathcal{N}}$ are briefly recalled in Section 2 . We introduce featured interpretations in Section 3 and show that featured interpretations provide a tight approximation to the standard semantics of DL-Lite. We also investigate the conditions for a set of featured interpretations to correspond to a DL-Lite KB. In Section 4 , we define two ontology revision operators based on featured models and two notions of featured-model distance, and discuss some interesting properties. The rationality of our revision operators are further justified using (adapted) AGM postulates. In Section 5 , we present the complexity results and provide computation algorithms for the revision operators. We introduce the state-of-the-art in ontology revision and compare our approach with some existing ones in Section 6 . Finally, Section 7 concludes the paper. Some tedious technical proofs are left in the appendix at the end of the paper. 


\section{DL-LITE FAMILY}

In this section, we briefly recall some basics of DL-Lite that will be used in this paper. Also, through an example (Example 2.1), we demonstrate that a simple ontology in DL-Lite can have an infinite number of models and all of the models are infinite first-order structures.

\subsection{Syntax of DL-Lite}

A signature is a (possibly infinite) set $\mathcal{S}=\mathcal{S}_{C} \cup \mathcal{S}_{R} \cup \mathcal{S}_{I} \cup \mathcal{S}_{N}$ where $\mathcal{S}_{C}, \mathcal{S}_{R}, \mathcal{S}_{I}$ and $\mathcal{S}_{N}$ are mutually disjoint. $\mathcal{S}_{C}$ is a set of atomic concepts, $\mathcal{S}_{R}$ is a set of atomic roles, $\mathcal{S}_{I}$ is a set of individual names, and $\mathcal{S}_{N}$ is a set of natural numbers. We assume the number 1 is always in $\mathcal{S}_{N}$. $\top$ and $\perp$ are special symbols and are neither atomic concepts nor atomic roles. Complex concepts and roles in DL-Lite $\mathcal{b o o l}_{\text {Nol }}^{\mathcal{N}}$ can be constructed from the atomic ones as follows:

$$
\begin{aligned}
& R \longrightarrow P \mid P^{-} \\
& B \longrightarrow \top|A| \geqslant n R \\
& C \longrightarrow B|\neg C| C_{1} \sqcap C_{2}
\end{aligned}
$$

where $n \in \mathcal{S}_{N}, A \in \mathcal{S}_{C}$ and $P \in \mathcal{S}_{R}$. B is called a basic concept and $C$ is called a general concept or simply concept. $B C_{\mathcal{S}}$ denotes the set of all basic concepts on $\mathcal{S}$. We write $\perp$ as a shorthand for $\neg \top, \exists R$ for $\geqslant 1 R$, $\leqslant n R$ for $\neg(\geqslant n+1 R)$, and $C_{1} \sqcup C_{2}$ for $\neg\left(\neg C_{1} \sqcap \neg C_{2}\right)$. We use $\mathcal{S}_{R}^{-}$to denote $\left\{P^{-} \mid P \in \mathcal{S}_{R}\right\}$. For each $P \in \mathcal{S}_{R}$, let $R^{+}=P$ whenever $R=P$ or $R=P^{-}$.

A TBox $\mathcal{T}$ is a finite set of concept inclusions of the form $C_{1} \sqsubseteq C_{2}$ with $C_{1}$ and $C_{2}$ being general concepts. An ABox $\mathcal{A}$ is a finite set of membership assertions of the forms $C(a), P(a, b)$, and $\neg P(a, b)$, where $a, b \in \mathcal{S}_{I}$. We call $C(a)$ a concept assertion and $P(a, b)$ or $\neg P(a, b)$ a role assertion. For simplicity, we disallow assertions of the form $P^{-}(a, b)$ in the ABoxes; however, we may write $R(a, b)$ where $R=P^{-}$is a inverse role, meaning $P(b, a)$. Note that some literature on DL-Lite consider concept assertions only of the form $A(a)$ with $A$ being an atomic concept name. Allowing general concepts in concept assertions $C(a)$ does not necessarily extend the expressive power, as $C(a)$ can be simulated by assertion $A_{C}(a)$ and inclusion $A_{C} \sqsubseteq C$ where $A_{C}$ is a fresh atomic concept name. An axiom is either a concept inclusion or a (concept or role) assertion. A knowledge base $(\mathrm{KB})$ is a pair $\mathcal{K}=\langle\mathcal{T}, \mathcal{A}\rangle$. In this paper, a DL ontology is a DL KB.

\subsection{Semantics of DL-Lite}

The semantics of a DL-Lite KB is given by interpretations. An interpretation $\mathcal{I}$ is a pair $\left(\Delta^{\mathcal{I}}, .^{\mathcal{I}}\right)$, where $\Delta^{\mathcal{I}}$ is a non-empty set called the domain and ${ }^{\mathcal{I}}$ is an interpretation function which associates each atomic concept $A$ with a subset $A^{\mathcal{I}}$ of $\Delta^{\mathcal{I}}$, each atomic role $P$ with a binary relation $P^{\mathcal{I}} \subseteq$ $\Delta^{\mathcal{I}} \times \Delta^{\mathcal{I}}$, and each individual $a$ with an element $a^{\mathcal{I}}$ of $\Delta^{\mathcal{I}}$ such that $a^{\mathcal{I}} \neq b^{\mathcal{I}}$ for each pair of $a, b \in \mathcal{S}_{I}$ (unique name assumption).

The interpretation function $\cdot{ }^{\mathcal{I}}$ can be extended to general concept descriptions:

$$
\begin{aligned}
\left(P^{-}\right)^{\mathcal{I}} & =\left\{(d, e) \mid(e, d) \in P^{\mathcal{I}}\right\} \\
(\neg P)^{\mathcal{I}} & =\Delta \times \Delta-P^{\mathcal{I}}, \\
(\geqslant n R)^{\mathcal{I}} & =\left\{d||\left\{e \mid(d, e) \in R^{\mathcal{I}}\right\} \mid \geq n\right\} \\
(\neg C)^{\mathcal{I}} & =\Delta^{\mathcal{I}}-C^{\mathcal{I}} \\
\left(C_{1} \sqcap C_{2}\right)^{\mathcal{I}} & =C_{1}^{\mathcal{I}} \cap C_{2}^{\mathcal{I}}
\end{aligned}
$$

An interpretation $\mathcal{I}$ satisfies inclusion $C_{1} \sqsubseteq C_{2}$ if $C_{1}^{\mathcal{I}} \subseteq C_{2}^{\mathcal{I}} ; \mathcal{I}$ satisfies assertion $C(a)$ if $a^{\mathcal{I}} \in$ $C^{\mathcal{I}} ; \mathcal{I}$ satisfies assertion $P(a, b)$ if $\left(a^{\mathcal{I}}, b^{\mathcal{I}}\right) \in P^{\mathcal{I}} . \mathcal{I}$ satisfies TBox $\mathcal{T}$ (or ABox $\mathcal{A}$ ) if $\mathcal{I}$ satisfies each inclusion in $\mathcal{T}$ (resp., each assertion in $\mathcal{A}$ ). $\mathcal{I}$ is a model of a $\mathrm{KB}\langle\mathcal{T}, \mathcal{A}\rangle$, if $\mathcal{I}$ satisfies both $\mathcal{T}$ and $\mathcal{A}$. We use $\bmod (\mathcal{K})$ to denote the set of models of $\mathrm{KB} \mathcal{K} \cdot \operatorname{sig}(\mathcal{K})$ is the signature of $\mathcal{K}$.

$\mathrm{A} \mathrm{KB} \mathcal{K}$ is consistent if it has at least one model. A concept or role $E$ is satisfiable w.r.t. $\mathcal{K}$ if there exists a model $\mathcal{I}$ of $\mathcal{K}$ such that $E^{\mathcal{I}} \neq \emptyset$. A KB $\mathcal{K}$ is coherent if each atomic concept and each atomic role are satisfiable w.r.t. $\mathcal{K}$.

Two KBs $\mathcal{K}_{1}, \mathcal{K}_{2}$ that have the same models are said to be equivalent, denoted $\mathcal{K}_{1} \equiv \mathcal{K}_{2}$. A KB $\mathcal{K}$ entails an inclusion or assertion $\alpha$, denoted $\mathcal{K} \models \alpha$, if all models of $\mathcal{K}$ satisfy $\alpha$. Subsumption and 
instance checking are standard reasoning tasks of deciding whether $\mathcal{K}=\alpha$ with $\alpha$ being a concept inclusion and a membership assertion, respectively.

Given a set $\mathbb{M}$ of interpretations, a DL language $\mathcal{L}$ and a signature $\mathcal{S}$, in most cases there does not exist a KB $\mathcal{K}$ expressed in $\mathcal{L}$ over $\mathcal{S}$ such that the models of $\mathcal{K}$ are exactly $\mathbb{M}$. To tackle this inexpressibility problem, a notion of best approximation is introduced in [Giacomo et al. 2007]. A $\mathrm{KB} \mathcal{K}$ in $\mathcal{L}$ is said to be a maximal approximation (MA) of $\mathbb{M}$ in $\mathcal{L}$ over $\mathcal{S}$ if $(1) \operatorname{sig}(\mathcal{K}) \subseteq \mathcal{S}$ and $\mathbb{M} \subseteq \bmod (\mathcal{K})$, and (2) there exists no KB $\mathcal{K}^{\prime}$ in $\mathcal{L}$ satisfying (1) such that $\bmod \left(\mathcal{K}^{\prime}\right) \subset \bmod (\mathcal{K})$. It is shown in [Giacomo et al. 2007] that MA may not exist for some DLs. However, we can show that MAs always exist in DL-Lite ${ }_{\text {bool }}$. When it exists, the MA of $\mathbb{M}$ is unique up to KB equivalence. If $\mathrm{KB} \mathcal{K}$ is the MA of $\mathbb{M}$ in $\mathcal{L}$ over $\mathcal{S}$, then for any inclusion or assertion $\alpha$ in $\mathcal{L}$ over $\mathcal{S}, \mathcal{K}=\alpha$ if and only if all interpretations in $\mathbb{M}$ satisfy $\alpha$.

\subsection{Infinity of DL-Lite Models}

Before defining the concept of features, we first look into the cause of the infinity of classical models for DL-Lite. Since we only consider finite signatures, the cause of the infinity of models is the possibly infinite interpretation domain. To avoid infinite models, we could consider only models with finite domains. However, DL-Lite $\mathrm{b}_{\mathrm{N} o o l}^{\mathcal{N}}$ does not enjoy the finite model property, i.e., there are simple DL-Lite KBs that have only infinite models as shown in an example from |Calvanese et al. 2006]. In particular, all models of the following KB $\mathcal{K}$ must have infinite domain.

Example 2.1. Consider the $\mathrm{KB} \mathcal{K}=\langle\mathcal{T}, \mathcal{A}\rangle$, where

$$
\begin{aligned}
& \mathcal{T}=\left\{A \sqsubseteq \exists P, B \sqsubseteq \exists P, \exists P^{-} \sqsubseteq B, A \sqcap B \sqsubseteq \perp, \geq 2 P^{-} \sqsubseteq \perp\right\} \\
& \mathcal{A}=\{A(a), P(a, b)\} .
\end{aligned}
$$

In fact, an infinite model $\mathcal{I}$ of $\mathcal{K}$ can be defined as follows:

$-\Delta^{\mathcal{I}}=\left\{a, b, d_{1}, d_{2}, d_{3} \ldots\right\}, a^{\mathcal{I}}=a$ and $b^{\mathcal{I}}=b ;$

$-A^{\mathcal{I}}=\{a\}$ and $B^{\mathcal{I}}=\left\{b, d_{1}, d_{2}, d_{3} \ldots\right\} ;$ and

$-P^{\mathcal{I}}=\left\{(a, b),\left(b, d_{1}\right),\left(d_{1}, d_{2}\right), \ldots,\left(d_{i}, d_{i+1}\right), \ldots\right\}$.

In the above example, $B \sqsubseteq \exists P$ says that each member of class $B$ has a $P$-successor; $\exists P^{-} \sqsubseteq$ $B$ then enforces each such $\bar{P}$-successor to be in $B$ and thus to have its $P$-successor; and finally $A \sqcap B \sqsubseteq \perp$ and $\geq 2 P^{-} \sqsubseteq \perp$ rule out loops. Thus, only an interpretation with an infinite chain of $P$-successors can be a model of $\mathcal{K}$. On the other hand, however, an important observation is that the (infinitely many) domain elements $d_{1}, d_{2}, \ldots$ are not crucial for standard subsumption and instance checking reasoning problems. Indeed, subsumption can be characterized by sets of basic concepts, called types, which will be defined in the next section; and instance checking can be characterised by interpretations on named individuals, called Herbrand sets.

\section{FEATURED INTERPRETATIONS FOR DL-LITE}

In this section, we introduce the notion of featured interpretations/models, which provides an alternative semantic characterization for DL-Lite. An advantage of featured models over models is that the number of all featured models for a DL-Lite KB is finite and each featured model is finite as well. These finiteness properties make it possible to recast key approaches to belief change for classical propositional logic into DL-Lite.

In the following discussions, it is often sufficient to consider a finite signature, still denoted $\mathcal{S}$, such that all the atomic concepts, atomic role names, individual names and numbers occurring in the input KBs are contained in $\mathcal{S}$. In Section 3.5, we will consider extensions of $\mathcal{S}$ with finitely many auxiliary role names not occurring in the input KBs, and the extended signatures are still finite. Hence, in what follows, we only consider finite signatures. 


\subsection{Types in DL-Lite}

A type for DL-Lite [Kontchakov et al. 2008, Zhuang et al. 2014] is defined as a set of basic concepts that satisfies a condition on numerical restrictions.

Definition 3.1. For a finite signature $\mathcal{S}$, an $\mathcal{S}$-type (or simply a type) $\tau$ is a set of basic concepts over $\mathcal{S}$, i.e., $\tau \subseteq B C_{\mathcal{S}}$, such that $\top \in \tau$, and for any $m, n \in \mathcal{S}_{N}$ with $m<n, \geqslant n R \in \tau$ implies $\geqslant m R \in \tau$.

Note that $\perp$ is not a basic concept and is not contained in any type. Also, as $T$ occurs in every type, we often omit it for simplicity.

Example 3.2. Let $\mathcal{S}_{C}=\{A, B\}, \mathcal{S}_{R}=\{P\}$, and $\mathcal{S}_{N}=\{1,2\}$. Then $\tau=\{A, \exists P, \geqslant 2 P$, $\left.\exists P^{-}\right\}$is a type. However, $\left\{A, \geqslant 2 P, \exists P^{-}\right\}$is not a type, as the presence of $\geqslant 2 P$ requires $\exists P$ to be included.

Clearly, as the number of basic concepts over $\mathcal{S}$ is finite, the number of $\mathcal{S}$-types is also finite. For an interpretation $\mathcal{I}$, each domain element $d \in \Delta^{\mathcal{I}}$ induces a unique type. Define $\tau^{\mathcal{I}}(d)=\{B \in$ $\left.B C_{\mathcal{S}} \mid d \in B^{\mathcal{I}}\right\}$. We say that $d$ induces $\tau^{\mathcal{I}}(d)$ in $\mathcal{I}$. Hence, the elements in $\Delta^{\mathcal{I}}$ can be classified using the types they induce. Two elements are type-equivalent if they induce the same type. In this way, all the (possibly infinite) elements are grouped into a finite number of type-equivalent classes.

The following result shows that type-equivalent domain elements cannot be distinguished by the concept membership, i.e., they all belong to the same concepts.

LEMMA 3.3. Given an interpretation $\mathcal{I}$ over $\mathcal{S}$, for $d_{1}, d_{2} \in \Delta^{\mathcal{I}}$, if $d_{1}$ and $d_{2}$ are typeequivalent, then $d_{1} \in C^{\mathcal{I}}$ iff $d_{2} \in C^{\mathcal{I}}$ for each concept $C$ over $\mathcal{S}$.

By Lemma 3.3 we can further conclude that type-equivalent domain elements cannot be distinguished by concept inclusions. Thus, we can use type $\tau$ as a representative for all the domain elements that induce $\tau$. In what follows, we formally define the satisfaction of concepts and concept inclusions using types.

The satisfiability of a concept in a type can be defined in a standard way: A type $\tau$ satisfies a basic concept $B$ if $B \in \tau ; \tau$ satisfies $\neg C$ if $\tau$ does not satisfy $C$; and $\tau$ satisfies $C_{1} \sqcap C_{2}$ if $\tau$ satisfies both $C_{1}$ and $C_{2}$.

LEMMA 3.4. Let $C$ be any concept over $\mathcal{S}$. Given an interpretation $\mathcal{I}$ over $\mathcal{S}, d \in C^{\mathcal{I}}$ iff $\tau^{\mathcal{I}}(d)$ satisfies $C$.

Define that a type $\tau$ satisfies concept inclusion $C_{1} \sqsubseteq C_{2}$ if $\tau$ satisfies concept $\neg C_{1} \sqcup C_{2}$. A type $\tau$ satisfies a TBox $\mathcal{T}$ if $\tau$ satisfies every inclusion in $\mathcal{T}$. Although the definition of a type satisfying a TBox looks propositional (with basic concepts seen as propositional atoms, TBoxes as propositional theories, and types as propositional interpretations), a type is different from a propositional interpretation. In particular, the logical connections between basic concepts $\geqslant n R$ and $\geqslant n+k R$, and between $\exists P$ and $\exists P^{-}$, require some special consideration. The former is addressed in the definition of types, and the latter, as we will show, needs to be specially considered only when role $P$ is unsatisfiable w.r.t. the TBox.

A TBox $\mathcal{T}$ is called role coherent if each role $P$ in $\mathcal{T}$ is satisfiable, i.e., for each $P \in \mathcal{S}_{R}$, a model $\mathcal{I}$ of $\mathcal{T}$ exists such that $P^{\mathcal{I}} \neq \emptyset$. We show that types (alone) characterize the semantics of role coherent TBoxes in a propositional manner. In particular, the concept satisfiability and subsumption for role coherent TBoxes that are defined by classical DL models can be equally defined by types. A concept $C$ is type-satisfiable w.r.t. a TBox $\mathcal{T}$ if there exists a type satisfying both $\mathcal{T}$ and $C$.

PROPOSITION 3.5. Let $C$ be a concept and $\mathcal{T}$ be a role coherent TBox over $\mathcal{S}$. $C$ is satisfiable w.r.t. $\mathcal{T}$ iff $C$ is type-satisfiable w.r.t. $\mathcal{T}$.

If $\mathcal{T}$ is not role coherent, by Lemma 3.4 the "only if" direction of Proposition 3.5 still holds, whereas the "if" direction does not necessarily hold. For example, let $\mathcal{T}=\{\exists P \sqsubseteq \perp\}$, then 
concept $\exists P^{-}$is clearly unsatisfiable w.r.t. $\mathcal{T}$, yet it is type-satisfiable w.r.t. $\mathcal{T}$ witnessed by the type $\left\{\exists P^{-}\right\}$. As we will see in Section 3.3, for such a (not necessarily role coherent) TBox $\mathcal{T}$, sets of types instead of single types need to be used to characterise the semantics of $\mathcal{T}$.

As concept subsumption can be reduced to concept (un)satisfiability, we also have the following immediate consequence of Proposition 3.5 For a TBox $\mathcal{T}$ and a concept inclusion $C \sqsubseteq D$, we define $\mathcal{T} \models_{t} C \sqsubseteq D$ if all the types $\tau$ satisfying $\mathcal{T}$ also satisfy $C \sqsubseteq D$.

COROllary 3.6. Let $\mathcal{T}$ be a role coherent TBox. For any two concepts $C$ and $D$ over $\mathcal{S}$, $\mathcal{T} \models C \sqsubseteq D$ iff $\mathcal{T} \models_{t} C \sqsubseteq D$.

\subsection{Herbrand Sets}

Types do not refer to individuals, and they are insufficient to capture the semantics of ABoxes. ABoxes assert membership of individuals. Hence, we need to extend types and introduce a semantic characterization for ABoxes, in which each interpretation needs to specify the types and the role memberships of the individuals. We adopt Herbrand interpretations in first order logic, and show that they are sufficient for instance checking.

Definition 3.7. For a finite signature $\mathcal{S}$, an $\mathcal{S}$-Herbrand set (or simply a Herbrand set) $\mathcal{H}$ is a finite set of basic assertions of the form $B(a)$ or $P(a, b)$, where $a, b \in \mathcal{S}_{I}, B \in B C_{\mathcal{S}}$ and $P \in \mathcal{S}_{R}$, satisfying the following three conditions:

(1) For each $a \in \mathcal{S}_{I}, \top(a) \in \mathcal{H}$, and $\geqslant n R(a) \in \mathcal{H}$ implies $\geqslant m R(a) \in \mathcal{H}$ for each $R \in \mathcal{S}_{R} \cup \mathcal{S}_{R}^{-}$ and each pair $m, n \in \mathcal{S}_{N}$ with $m<n$.

(2) For each $P \in \mathcal{S}_{R}$ and each $m \in \mathcal{S}_{N}, P\left(a, b_{i}\right) \in \mathcal{H}(i=1, \ldots, n)$ with $n \geq m$ implies $\geqslant m P(a) \in \mathcal{H}$.

(3) For each $P \in \mathcal{S}_{R}$ and each $m \in \mathcal{S}_{N}, P\left(b_{i}, a\right) \in \mathcal{H}(i=1, \ldots, n)$ with $n \geq m$ implies $\geqslant m P^{-}(a) \in \mathcal{H}$.

By Condition (1) in Definition 3.7 for each individual $a \in \mathcal{S}_{I}$, the set $\tau^{\mathcal{H}}(a)=\{B \mid B(a) \in \mathcal{H}\}$ is a type, and it is referred to as the type of $a$ in $\mathcal{H}$. Taking $\mathcal{H}$ as a Herbrand interpretation where each individual name is interpreted as itself, by Lemma 3.4. $\tau^{\mathcal{H}}(a)$ provides a complete interpretation on the concept membership of $a$. Conditions (2) and (3) say that the interpretation on concept membership must be consistent with that on role membership. In particular, if an individual $a$ has at least $m$ explicitly specified $P$-successors (or resp., $P$-predecessors) for some $m \in \mathcal{S}_{N}$, then $a$ must be a member of concept $\geqslant m P\left(\right.$ resp., $\left.\geqslant m P^{-}\right)$. Note that we adopt unique name assumption (UNA) here, and treat individuals with different names as distinct ones.

A Herbrand set $\mathcal{H}$ (viewed as a Herbrand interpretation) contains the complete interpretation about (only) the named individuals. For instance, the absence of $B(a)$ in $\mathcal{H}$, with $B$ being a basic concept and $a$ being a named individual, means that $a$ is not a member of $B$, or equivalently, $a$ is a member of $\neg B$. Note that we are not making the domain closure assumption, in which case the domain of the interpretation consists of only named individuals. An interpretation that induces a Herbrand set may contain unnamed domain elements, and the Herbrand set simply does not say anything about them.

Since $T(a)$ is in every Herbrand set and $a \in \mathcal{S}_{I}$, for simplicity, we will omit it in examples. We use $C A(a, \mathcal{H})$ to denote the set of concept assertions containing $a$ in $\mathcal{H}$, and use $R A(\mathcal{H})$ to denote the set of role assertions in $\mathcal{H}$. Although role assertions of the form $P^{-}(a, b)$ are not allowed in a Herbrand set, we will sometimes write $P^{-}(a, b) \in \mathcal{H}$ meaning $P(b, a) \in \mathcal{H}$ for simplicity.

Now we define the satisfaction of membership assertions in terms of Herbrand sets. We say that a Herbrand set $\mathcal{H}$ satisfies concept assertion $C(a)$ if $\tau^{\mathcal{H}}(a)$ satisfies concept $C$. Herbrand set $\mathcal{H}$ satisfies role assertion $P(a, b)$ if $P(a, b)$ is in $\mathcal{H}$, and $\neg P(a, b)$ if $P(a, b)$ is not in $\mathcal{H}$. Herbrand set $\mathcal{H}$ satisfies an $\mathrm{ABox} \mathcal{A}$ if $\mathcal{H}$ satisfies every assertion in $\mathcal{A}$. For an $\mathrm{ABox} \mathcal{A}$ and an (concept or role) assertion $\alpha$, we define $\mathcal{A} \models_{h} \alpha$ if all Herbrand sets $\mathcal{H}$ satisfying $\mathcal{A}$ also satisfy $\alpha$. 
Proposition 3.8. Let $\mathcal{A}$ be an ABox, $C(a)$ be a concept assertion, and $P(a, b)$ be a role assertion over $\mathcal{S}$. We have $\mathcal{A} \models C(a)$ iff $\mathcal{A} \models_{h} C(a)$, and $\mathcal{A} \models P(a, b)$ iff $\mathcal{A} \models_{h} P(a, b)$.

\subsection{Featured Interpretations of DL-Lite}

To provide an alternative semantic characterization for DL-Lite KBs, we could use pairs $\langle\tau, \mathcal{H}\rangle$, where $\tau$ is a type and $\mathcal{H}$ is a Herbrand set, to replace classical interpretations for DLs, and define that $\langle\tau, \mathcal{H}\rangle$ satisfies $\operatorname{KB}\langle\mathcal{T}, \mathcal{A}\rangle$ if $\tau$ satisfies $\mathcal{T}$ and $\mathcal{H}$ satisfies $\mathcal{A}$. The resulting satisfaction relation should guarantee that $\mathcal{K}$ is consistent iff there exists some pair $\langle\tau, \mathcal{H}\rangle$ satisfying $\mathcal{K}$. However, the following examples show that it is not the case.

Example 3.9. (1) Given a TBox $\mathcal{T}=\left\{\top \sqsubseteq \exists P, \exists P^{-} \sqsubseteq \perp\right\}$, it is clear that $\mathcal{T}$ is inconsistent. However, there is a type $\tau=\{\exists P\}$ satisfying $\overline{\mathcal{T}}$.

(2) Let $\mathcal{K}=\langle\mathcal{T}, \mathcal{A}\rangle$ where $\mathcal{T}=\{A \sqsubseteq \neg B\}$ and $\mathcal{A}=\{A(a), B(a)\}$. Note that $\mathcal{K}$ is inconsistent while $\mathcal{T}$ and $\mathcal{A}$ are respectively consistent. Take the type $\tau=\{A\}$ and the Herbrand set $\mathcal{H}=$ $\{A(a), B(a)\}$. Then $\tau$ satisfies $\mathcal{T}$ and $\mathcal{H}$ satisfies $\mathcal{A}$.

Example 3.9 (1) shows that a single type is insufficient to reflect the logical connection between $\exists P$ and $\exists P^{-}$, and hence the simple characterization does not work for non-role coherent TBoxes. Intuitively, $\exists P$ is satisfiable w.r.t. $\mathcal{T}$ if and only if $\exists P^{-}$is satisfiable w.r.t. $\mathcal{T}$. To express this condition, it often requires at least two types satisfying $\exists P$ and $\exists P^{-}$respectively. Example 3.9 (2) shows that with a pair $\langle\tau, \mathcal{H}\rangle$ of a single type and a Herbrand set, it is difficult to express the logical connection between the TBox and the ABox of a KB.

Generating models [Konev et al. 2011] have been used to approximate canonical models of DLLite KBs with finite structures, yet generating models cannot be directly adopted for our purpose. In general, it is unclear how to re-construct $\mathrm{KBs} / \mathrm{TB}$ oxes from generating models (needed for computing revision results like in Algorithm 2 2 . For example, let $\mathcal{T}=\{A \sqsubseteq B\}$ and $\mathcal{A}=\{A(a)\}$, then $\langle\mathcal{T}, \mathcal{A}\rangle$ has the unique generating model $\{A(a), B(a)\}$, which does not contain the types $\tau_{1}=\{B\}$ and $\tau_{2}=\emptyset$ that are needed for fully capturing the subsumption $A \sqsubseteq B$.

We address this issue by using a set of types (instead of a single type) in our characterization. Using sets of types as semantic characterizations of DL-Lite TBoxes is also suggested in [Kontchakov et al. 2008]. Figure 1 shows how a classical DL model is approximated with a set of types and a Herbrand set. A DL model $\mathcal{I}$ can be seen as a (generally infinite) graph $\left(\Delta^{\mathcal{I}},\left\{(d, e) \mid\right.\right.$ there exists $P \in \mathcal{S}_{R}$ s.t. $\left.\left.(d, e) \in P^{\mathcal{I}}\right\}\right)$, where each node is a domain element and is labelled with a type, and each edge is labelled with a (set of) role name(s). A black node corresponds to (the interpretation of) a named individual. In our new semantic characterisation, we use an approximation of $\mathcal{I}$ which consists of the set $\Xi$ of all labelling types and the Herbrand set $\mathcal{H}$ obtained by restricting $\mathcal{I}$ to assertions about named individuals. Such an approximation abstracts from the interpretation some critical features w.r.t. concept subsumption (Corollary 3.6) and instance checking (Proposition 3.8), and is independent of the interpretation domain.

We first introduce the definition of featured interpretations, and then show that they are tight approximations of classical DL interpretations.

Definition 3.10 (Featured Interpretaitons). For a finite signature $\mathcal{S}$, an $\mathcal{S}$-featured interpretation (or simply a featured interpretation) is a pair $\mathcal{F}=\langle\Xi, \mathcal{H}\rangle$, where $\Xi$ is a non-empty set of $\mathcal{S}$-types and $\mathcal{H}$ is an $\mathcal{S}$-Herbrand set, satisfying the following two conditions:

(1) Concept $\exists P$ occurs in $\Xi$ iff concept $\exists P^{-}$occurs in $\Xi$ for each $P \in \mathcal{S}_{R}$.

(2) Type $\tau^{\mathcal{H}}(a) \in \Xi$ for each $a \in \mathcal{S}_{I}$.

Intuitively, in a featured interpretation $\langle\Xi, \mathcal{H}\rangle, \Xi$ consists of the types induced by the elements in the domain of interest, and $\mathcal{H}$ specifies the membership of all named individuals. Condition (1) of Definition 3.10 says that if there is a type $\tau$ in $\Xi$ containing $\exists P$ for some role $P$, then there must be a type $\tau^{\prime}$ in $\Xi$ containing $\exists P^{-}$. Note that $\tau$ and $\tau^{\prime}$ are not necessarily different. Condition (2) says that the types induced by individuals must all belong to $\Xi$. This ensures the interpretation of membership 


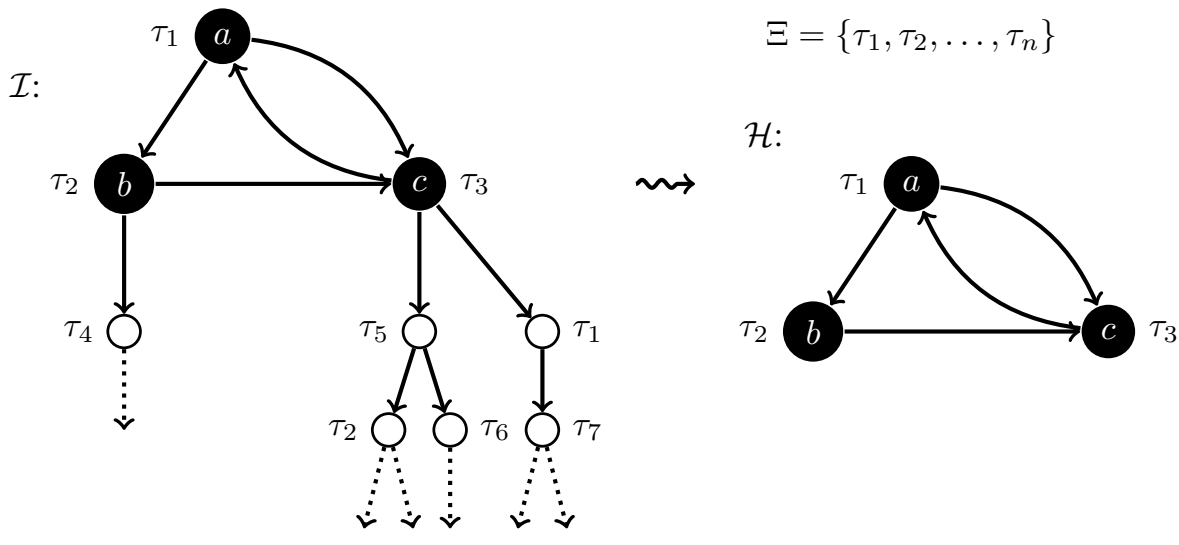

Fig. 1. An approximation of an interpretation.

in the ABox to be consistent with the terminological constraints in the TBox. Conditions (1) and (2) address the problems shown in Example 3.9 (1) and (2), respectively. A featured interpretation is not a first-order interpretation and is independent from the domain, which has two advantages: first, finite featured models exist for TBoxes that have no finite DL/first-order models (as in Example 2.1); and secondly, as we will see later, it is more convenient to define distance between featured models when unnamed individuals in the domain are disregarded.

From the definition, we can see that a featured interpretation is a finite structure, and the number of all featured interpretations is also finite. This is mainly due to the fact that we only consider finite signatures. Given a finite signature $\mathcal{S}$, the number of basic concepts over $\mathcal{S}$ is finite. Consider the first part $\Xi$ of an $\mathcal{S}$-featured interpretation, $\Xi$ is a subset of the power set of $B C_{\mathcal{S}}$. Thus, $\Xi$ is always finite, and the number of all possible $\Xi$ is also finite. Consider the second part $\mathcal{H}$, it is finite because (1) the number of individuals in $\mathcal{S}$ is finite; (2) for each named individual $a$, the type of $a, \tau^{\mathcal{H}}(a)$ is finite; and (3) the number of role membership assertions for individuals is finite. Moreover, there are also only a finite number of possible Herbrand sets.

In what follows, we show a connection between (possibly infinite) DL interpretations and (finite) featured interpretations. First, each interpretation $\mathcal{I}$ uniquely induces an $\mathcal{S}$-featured interpretation $\mathcal{F}_{\mathcal{I}}=\langle\Xi, \mathcal{H}\rangle$ defined as follows:

$$
\begin{aligned}
\Xi_{\mathcal{I}}= & \left\{\tau^{\mathcal{I}}(d) \mid d \in \Delta^{\mathcal{I}}\right\} \text { and } \\
\mathcal{H}_{\mathcal{I}}= & \left\{B(a) \mid a \in \mathcal{S}_{I}, B \in B C_{\mathcal{S}}, a^{\mathcal{I}} \in B^{\mathcal{I}}\right\} \cup \\
& \left\{P(a, b) \mid a, b \in \mathcal{S}_{I}, P \in \mathcal{S}_{R}, \text { and }\left(a^{\mathcal{I}}, b^{\mathcal{I}}\right) \in P^{\mathcal{I}}\right\} .
\end{aligned}
$$

$\mathcal{F}_{\mathcal{I}}$ is referred to as the featured interpretation induced by $\mathcal{I}$.

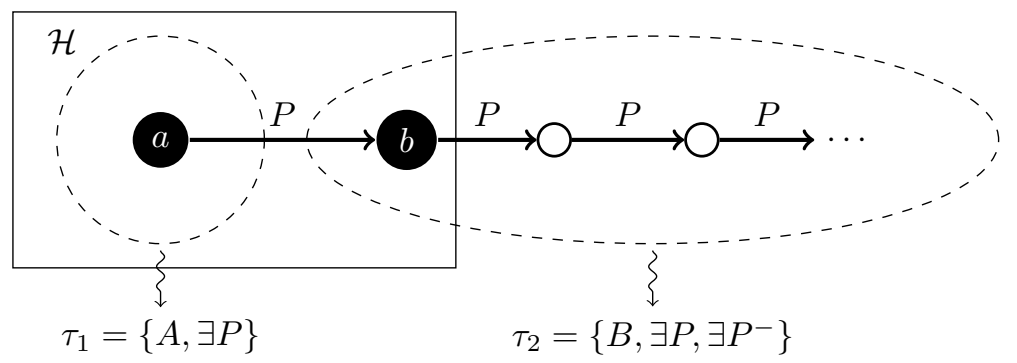

Fig. 2. A finite featured interpretation induced by an infinite interpretation. 
Example 3.11. (Cont. Example 2.1) Take $\mathcal{S}=\operatorname{sig}(\mathcal{K})=\{A, B, P, 1,2, a, b\}$. A (finite) featured interpretation induced by $\mathcal{I}$ is $\mathcal{F}_{\mathcal{I}}=\left\langle\Xi_{\mathcal{I}}, \mathcal{H}_{\mathcal{I}}\right\rangle$, where $\Xi_{\mathcal{I}}=\left\{\tau_{1}, \tau_{2}\right\}$ with $\tau_{1}=\{A, \exists P\}$ and $\tau_{2}=\left\{B, \exists P, \exists P^{-}\right\}$, and $\mathcal{H}_{\mathcal{I}}=\left\{A(a), \exists P(a), B(b), \exists P(b), \exists P^{-}(b), P(a, b)\right\}$. The model $\mathcal{I}$ and the featured interpretation $\mathcal{F}_{\mathcal{I}}$ can be visualised in Figure 2. Note that the elements of the domain $\Delta^{\mathcal{I}}$ are grouped into two classes by types. In particular, $a$ induces type $\tau_{1}$, and $b$ and all the $d_{i}$ 's $(i \geq 1)$ induce type $\tau_{2}$. Also, Herbrand set $\mathcal{H}_{\mathcal{I}}$ contains only membership assertions about individuals $a$ and $b$.

Each interpretation uniquely induces a featured interpretation. But in general, a featured interpretation can be induced by different interpretations.

Example 3.12. Let $\mathcal{S}=\{a, A, P, 1\}$, and $\mathcal{I}_{1}$ and $\mathcal{I}_{2}$ be two interpretations, where $\Delta^{\mathcal{I}_{1}}=$ $\Delta^{\mathcal{I}_{2}}=\left\{d_{a}, d_{1}, d_{2}\right\}, a^{\mathcal{I}_{1}}=a^{\mathcal{I}_{2}}=d_{a}$, and

$$
\begin{aligned}
A^{\mathcal{I}_{1}}=\left\{d_{a}\right\}, & P^{\mathcal{I}_{1}}=\left\{\left(d_{a}, d_{1}\right),\left(d_{1}, d_{a}\right),\left(d_{1}, d_{2}\right),\left(d_{2}, d_{2}\right)\right\} \\
A^{\mathcal{I}_{2}}=\left\{d_{a}, d_{1}\right\}, P^{\mathcal{I}_{2}} & =\left\{\left(d_{a}, d_{1}\right),\left(d_{1}, d_{2}\right),\left(d_{2}, d_{a}\right)\right\}
\end{aligned}
$$

Suppose $\tau_{1}=\left\{A, \exists P, \exists P^{-}\right\}$and $\tau_{2}=\left\{\exists P, \exists P^{-}\right\}$. Then in $\mathcal{I}_{1}, d_{a}$ induces $\tau_{1}, d_{1}$ and $d_{2}$ induce $\tau_{2}$, while in $\mathcal{I}_{2}, d_{a}$ and $d_{1}$ induce $\tau_{1}$, and $d_{2}$ induces $\tau_{2}$. Both $\mathcal{I}_{1}$ and $\mathcal{I}_{2}$ induce the same featured interpretation $\left\langle\left\{\tau_{1}, \tau_{2}\right\},\left\{A(a), \exists P(a), \exists P^{-}(a)\right\}\right\rangle$.

The next result shows that each featured interpretation is induced by an interpretation. The proof of the result is quite involved where we show a construction of classical DL models by unravelling the featured interpretations.

PROPOSITION 3.13. Given a featured interpretation $\mathcal{F}$, there always exists an interpretation $\mathcal{I}$ such that $\mathcal{F}_{\mathcal{I}}=\mathcal{F}$.

\subsection{Entailment via Featured Interpretations}

Like the classical concept of satisfaction, a satisfaction relation in terms of featured interpretations can also be defined, which naturally extends the satisfaction relations for types and Herbrand sets.

Definition 3.14. Given a featured interpretation $\mathcal{F}=\langle\Xi, \mathcal{H}\rangle$, then

$-\mathcal{F}$ satisfies a concept $C$ if there is a type in $\Xi$ satisfying $C$.

- $\mathcal{F}$ satisfies a concept inclusion $C \sqsubseteq D$ if each type $\tau \in \Xi$ satisfies $C \sqsubseteq D$.

- $\mathcal{F}$ satisfies an assertion $C(a)$ or $S(a, b)$ if $\mathcal{H}$ satisfies it.

$-\mathcal{F}$ is a featured model of a $\mathrm{KB} \mathcal{K}$ if $\mathcal{F}$ satisfies every concept inclusion and every membership assertion in $\mathcal{K} . \operatorname{FM}(\mathcal{K})$ is the set of featured models of $\mathcal{K}$.

As shown earlier, there is a close correspondence between DL interpretations and featured interpretations. Moreover, we want to show that, given a KB $\mathcal{K}$, such a correspondence also exists between the models of $\mathcal{K}$ and the featured models of $\mathcal{K}$. In particular, we show that the featured models of a KB are exactly those featured interpretations induced by the DL models of $\mathcal{K}$. The following properties of the satisfaction relation for featured interpretations show that the concept of featured interpretations intuitively characterizes consequence entailment relations for DL-Lite.

PROPOSITION 3.15. Given an interpretation $\mathcal{I}$, it holds that

(1) $\mathcal{I}$ satisfies $C \sqsubseteq D$ iff $\mathcal{F}_{\mathcal{I}}$ satisfies $C \sqsubseteq D$, for each pair of concepts $C, D$ over $\mathcal{S}$;

(2) $\mathcal{I}$ satisfies $C(\bar{a})$ iff $\mathcal{F}_{\mathcal{I}}$ satisfies $C(a)$, for each assertion $C(a)$ over $\mathcal{S}$;

(3) $\mathcal{I}$ satisfies $S(a, b)$ iff $\mathcal{F}_{\mathcal{I}}$ satisfies $S(a, b)$, for each assertion $S(a, b)$ over $\mathcal{S}$.

Proof Let $\mathcal{F}_{\mathcal{I}}=\langle\Xi, \mathcal{H}\rangle$.

(1). I satisfies $C_{1} \sqsubseteq C_{2}$ iff $\mathcal{I}$ satisfies $\top \sqsubseteq \neg C_{1} \sqcup C_{2}$, iff for each $d \in \Delta^{\mathcal{I}}, d \in\left(\neg C_{1} \sqcup C_{2}\right)^{\mathcal{I}}$, iff for each $d \in \Delta^{\mathcal{I}}, \tau^{\mathcal{I}}(\bar{d})$ satisfies $\neg C_{1} \sqcup C_{2}$ (Lemma 3.4. That is, for each $\tau \in \Xi, \tau$ satisfies $\neg C_{1} \sqcup C_{2}$ iff $\mathcal{F}_{\mathcal{I}}$ satisfies $C_{1} \sqsubseteq C_{2}$. 
(2). I satisfies $C(a)$ iff $a^{\mathcal{I}} \in C^{\mathcal{I}}$, iff $\tau\left(a^{\mathcal{I}}, \mathcal{I}\right)$ satisfies concept $C$ (Lemma 3.4). That is, as $\tau\left(a^{\mathcal{I}}, \mathcal{I}\right)=\tau^{\mathcal{H}}(a), \tau^{\mathcal{H}}(a)$ satisfies $C$ iff $\mathcal{F}_{\mathcal{I}}$ satisfies $C(a)$.

(3). This can be seen directly from the construction of $\mathcal{F}_{\mathcal{I}}$.

It is easy to see that Proposition 3.15 has the following corollary.

COROLLARY 3.16. For a $K B \mathcal{K}$ and an interpretation $\mathcal{I}$, $\mathcal{I}$ is a model of $\mathcal{K}$ iff $\mathcal{F}_{\mathcal{I}}$ is a featured model of $\mathcal{K}$.

By Proposition 3.13 and Corollary 3.16, the correspondence between the featured models of a $\mathrm{KB} \mathcal{K}$ and the classical models of $\mathcal{K}$ is stated as follows.

Proposition 3.17. For a $K B \mathcal{K}, \operatorname{FM}(\mathcal{K})=\left\{\mathcal{F}_{\mathcal{I}} \mid \mathcal{I} \in \bmod (\mathcal{K})\right\}$ and $\bmod (\mathcal{K})=\left\{\mathcal{I} \mid \mathcal{F}_{\mathcal{I}} \in\right.$ $\operatorname{FM}(\mathcal{K})\}$.

Proof For each $\mathcal{F} \in \mathrm{FM}(\mathcal{K})$, from Proposition 3.13 , there is an interpretation $\mathcal{I}$ such that $\mathcal{F}_{\mathcal{I}}=\mathcal{F}$. By Corollary 3.16, $\mathcal{I} \in \bmod (\mathcal{K})$. That is, $\mathcal{F} \in\left\{\mathcal{F}_{\mathcal{I}} \mid \mathcal{I} \in \bmod (\mathcal{K})\right\}$. Conversely, for each $\mathcal{F} \in\left\{\mathcal{F}_{\mathcal{I}} \mid \mathcal{I} \in \bmod (\mathcal{K})\right\}$, from Corollary 3.16, $\mathcal{F} \in \mathrm{FM}(\mathcal{K})$.

For each $\mathcal{I} \in \bmod (\mathcal{K})$, by Corollary 3.16. $\mathcal{F}_{\mathcal{I}} \in \operatorname{FM}(\mathcal{K})$. Conversely, for each $\mathcal{I}$ such that $\mathcal{F}_{\mathcal{I}} \in \mathrm{FM}(\mathcal{K})$, from Corollary $3.16, \mathcal{I} \in \bmod (\mathcal{K})$.

The following result shows that featured models also behave like classical models under union of KBs. This result is useful in the proofs.

Proposition 3.18. Let $\mathcal{K}_{1}$ and $\mathcal{K}_{2}$ be two KBs. Then, $\operatorname{FM}\left(\mathcal{K}_{1} \cup \mathcal{K}_{2}\right)=\operatorname{FM}\left(\mathcal{K}_{1}\right) \cap \operatorname{FM}\left(\mathcal{K}_{2}\right)$.

Proof For a featured model $\mathcal{F} \in \mathrm{FM}\left(\mathcal{K}_{1} \cup \mathcal{K}_{2}\right)$, by Proposition 3.17, there is a model $\mathcal{I}$ of $\mathcal{K}_{1} \cup \mathcal{K}_{2}$ that induces $\mathcal{F}$. Clearly, $\mathcal{I} \in \bmod \left(\mathcal{K}_{1}\right)$ and $\mathcal{I} \in \bmod \left(\mathcal{K}_{2}\right)$. From Proposition 3.17, $\mathcal{F} \in \operatorname{FM}\left(\mathcal{K}_{1}\right)$ and $\mathcal{F} \in \operatorname{FM}\left(\mathcal{K}_{2}\right)$.

Conversely, for a featured model $\mathcal{F}$ such that $\mathcal{F} \in \mathrm{FM}\left(\mathcal{K}_{1}\right) \cap \operatorname{FM}\left(\mathcal{K}_{2}\right)$, by Proposition 3.13 . there is a model $\mathcal{I}$ inducing $\mathcal{F}$. From Proposition 3.17 $\mathcal{I}$ is a model of both $\mathcal{K}_{1}$ and $\mathcal{K}_{2}$. That is, $\mathcal{I} \in \bmod \left(\mathcal{K}_{1} \cup \mathcal{K}_{2}\right)$. Thus, by Proposition 3.17, $\mathcal{F} \in \mathrm{FM}\left(\mathcal{K}_{1} \cup \mathcal{K}_{2}\right)$.

The entailment relation determined by featured models can also be defined in a standard way.

Definition 3.19. Given a $\mathrm{KB} \mathcal{K}$ and a concept inclusion or an assertion $\alpha, \mathcal{K} \models_{f} \alpha$ if all featured models in $\mathrm{FM}(\mathcal{K})$ satisfy $\alpha$.

Given two $\mathrm{KBs} \mathcal{K}_{1}$ and $\mathcal{K}_{2}, \mathcal{K}_{1} \models_{f} \mathcal{K}_{2}$ if $\operatorname{FM}\left(\mathcal{K}_{1}\right) \subseteq \operatorname{FM}\left(\mathcal{K}_{2}\right)$, and $\mathcal{K}_{1} \equiv_{f} \mathcal{K}_{2}$ if $\operatorname{FM}\left(\mathcal{K}_{1}\right)=$ $\operatorname{FM}\left(\mathcal{K}_{2}\right)$.

By Propositions 3.15 and 3.17, the entailment for KBs determined by featured models coincides with classical entailment.

THEOREM 3.20. For a $K B \mathcal{K}$ with $\operatorname{sig}(\mathcal{K}) \subseteq \mathcal{S}$,

$-\mathcal{K}$ is consistent iff $\mathcal{K}$ has a featured model;

$-\mathcal{K} \models C \sqsubseteq D$ iff $\mathcal{K} \models_{f} C \sqsubseteq D$ for each pair of concepts $C, D$ over $\mathcal{S}$;

$-\mathcal{K}=C(\bar{a})$ iff $\mathcal{K} \models_{f} C(a)$ for each assertion $C(a)$ over $\mathcal{S}$; and

$-\mathcal{K} \models S(a, b)$ iff $\mathcal{K}={ }_{f} S(a, b)$ for each assertion $S(a, b)$ over $\mathcal{S}$.

Proof The theorem follows directly from Propositions 3.15 and 3.17

From the above theorem, the following result is immediate.

THEOREM 3.21. For two KBs $\mathcal{K}_{1}, \mathcal{K}_{2}$,

$-\mathcal{K}_{1} \models \mathcal{K}_{2}$ iff $\mathcal{K}_{1} \models_{f} \mathcal{K}_{2}$,

$-\mathcal{K}_{1} \equiv \mathcal{K}_{2}$ iff $\mathcal{K}_{1} \equiv{ }_{f} \mathcal{K}_{2}$.

Proof This is a direct conclusion from Theorem 3.20 
The above results further justify the rationality of using featured models as an alternative semantic characterization for DL-Lite. Moreover, the advantage of featured models over classical models for DL-Lite is that the number of featured models of a given KB is always finite, and each featured model is also finite in structure.

We note that while the notion of the featured interpretations is defined only for DL-Lite, it can be generalized to more expressive DLs. Moreover, several important properties of featured interpretations for DL-Lite still hold for expressive DLs:

— The definition of featured interpretations (Definition 3.10), as an approximation of classical DL interpretations, is applicable to any DL. The induction relation between a DL interpretation and a featured interpretation remains the same, and Proposition 3.13 holds for any DL.

- The satisfaction relation in terms of featured interpretations (i.e., the first three items in Definition 3.14 for more expressive DLs can be defined as follows: $\mathcal{F}$ satisfies a concept $C$ if and only if there is an interpretation inducing $\mathcal{F}$ that satisfies $C$; and $\mathcal{F}$ satisfies a concept inclusion or assertion $\alpha$ if and only if every interpretation inducing $\mathcal{F}$ satisfies $\alpha$. For a $\mathrm{KB} \mathcal{K}$, the featured models of $\mathcal{K}$ (the fourth item in Definition 3.14) can be defined as the featured interpretations induced by the models of $\mathcal{K}$ (as the first half of Proposition 3.17). The entailment and equivalence relations remain the same as in Definition 3.19

- Using the above definitions, the first item of Theorem 3.20 holds in general. Proposition 3.15 and Theorem 3.20 still hold for all DL-Lite ${ }_{\text {bool }}^{\mathcal{N}}$ concept inclusions and assertions. For more expressive concept inclusions and assertions, the "if" directions of Proposition 3.15 and the "only if" direction of Theorem 3.20 hold. The "if" directions of Theorem 3.21 holds.

It would be useful to explore properties of featured interpretations and related techniques that hold for a class of DLs instead of a single DL. This issue will be left for future work.

\subsection{Expressibility of Featured Interpretations}

Before using featured interpretations to define $\mathrm{KB}$ change operators in DL-Lite, we need to consider another important question: Does any given set of featured interpretations always correspond to a DL-Lite KB? Formally, a set $\mathbb{F}$ of featured interpretations is axiomatizable in DL-Lite $\mathcal{N}_{\text {bool }}^{\mathcal{N}}$ if there exists a DL-Lite $\mathcal{L}_{\text {bool }}^{\mathcal{N}} \mathrm{KB} \mathcal{K}$ such that $\operatorname{FM}(\mathcal{K})=\mathbb{F}$. The following example shows that not every set of featured interpretations is axiomatizable in DL-Lite ${ }_{\text {bool }}^{\mathcal{N}}$.

Example 3.22. We show a set of featured interpretations $\left\{\mathcal{F}_{1}, \mathcal{F}_{2}\right\}$ that is not axiomatizable in DL-Lite ${ }_{\text {bool }}^{\mathcal{N}}$, where $\mathcal{F}_{1}=\left\langle\left\{\tau_{1}\right\}, \mathcal{H}_{1}\right\rangle, \mathcal{F}_{2}=\left\langle\left\{\tau_{2}\right\}, \mathcal{H}_{2}\right\rangle, \tau_{1}=\{A\}, \tau_{2}=\{B\}$, $\mathcal{H}_{1}=\{A(a), A(b)\}$, and $\mathcal{H}_{2}=\{B(a), B(b)\}$. To see why $\left\{\mathcal{F}_{1}, \mathcal{F}_{2}\right\}$ is not axiomatizable, suppose on the contrary that it is axiomatizable, that is, there exists a DL-Lite $\mathcal{N}_{\text {bool }} \mathrm{KB} \mathcal{K}$ such that $\operatorname{FM}(\mathcal{K})=\left\{\mathcal{F}_{1}, \mathcal{F}_{2}\right\}$. Yet $\mathcal{F}_{1}$ and $\mathcal{F}_{2}$ cannot be the only featured models of $\mathcal{K}$. Consider a third featured interpretation $\mathcal{F}=\left\langle\left\{\tau_{1}, \tau_{2}\right\}, \mathcal{H}\right\rangle$, where $\mathcal{H}=\{A(a), B(b)\}$; we claim that if both $\mathcal{F}_{1}$ and $\mathcal{F}_{2}$ are featured models of $\mathcal{K}$, then $\mathcal{F}$ must be a featured model of $\mathcal{K}$ and thus would be in $\operatorname{FM}(\mathcal{K})$ too. To see this, we show that in such a case every axiom in $\mathcal{K}$ will be satisfied by $\mathcal{F}$.

For each concept inclusion $C \sqsubseteq D$ in $\mathcal{K}$, by Definition 3.14, $C \sqsubseteq D$ is satisfied by both $\mathcal{F}_{1}$ and $\mathcal{F}_{2}$. Thus, $C \sqsubseteq D$ is satisfied by both $\tau_{1}$ and $\tau_{2}$. This implies that $C \sqsubseteq D$ is satisfied by $\mathcal{F}$. For each membership assertion $\alpha$ in $\mathcal{K}, \alpha$ can only be one of the four possible forms: $C(a), D(b), R(a, b)$ and $\neg R(a, b)$. We only show that if $\alpha$ is $C(a)$ then $\alpha$ is satisfied by $\mathcal{F}$, and the other three cases are similar. In fact, by Definition 3.14 $C(a)$ is satisfied by $\tau^{\mathcal{H}_{1}}(a)$, and hence, $C(a)$ is satisfied by $\tau^{\mathcal{H}}(a)=\tau^{\mathcal{H}_{1}}(a)$. Thus, $C(a)$ is satisfied by $\mathcal{F}$.

Although a set of featured interpretations may not be axiomatizable, there is a simple characterisation for the axiomatizability of featured interpretations, which is based on a closure operator of constructing featured interpretations as shown below. Recall that for any Herbrand set $\mathcal{H}, C A(a, \mathcal{H})$ is the set of concept assertions containing individual $a$ in $\mathcal{H}$ and $R A(\mathcal{H})$ is the set of role assertions in $\mathcal{H}$. Given a set of featured interpretations $\mathbb{F}=\left\{\left\langle\Xi_{i}, \mathcal{H}_{i}\right\rangle \mid 1 \leq i \leq n\right\}$, define $\bigoplus \mathbb{F}$ to 
be the set of all possible featured interpretations $\langle\Xi, \mathcal{H}\rangle$ that satisfy the following three conditions: (1) $\Xi \subseteq \bigcup_{1 \leq i \leq n} \Xi_{i}$; (2) for each $a \in \mathcal{S}_{I}, C A(a, \mathcal{H})=C A\left(a, \mathcal{H}_{i}\right)$ for some $i$ with $1 \leq i \leq n$; and (3) $\bigcap_{1 \leq i \leq n} R A\left(\mathcal{H}_{i}\right) \subseteq R A(\mathcal{H}) \subseteq \bigcup_{1 \leq i \leq n} R A\left(\mathcal{H}_{i}\right)$. Intuitively, the $\bigoplus$ operator ensures that for any DL-Lite $\mathcal{L}_{\text {bool }}^{\mathcal{N}} \mathrm{KB} \mathcal{K}$, if all the featured interpretations in $\mathbb{F}$ satisfy $\mathcal{K}$ then all the featured interpretations satisfying $\mathcal{K}$ is contained in $\bigoplus \mathbb{F}$.

Example 3.23 (Cont. Example 3.22). For each featured interpretation $\langle\Xi, \mathcal{F}\rangle$ in $\bigoplus\left\{\mathcal{F}_{1}, \mathcal{F}_{2}\right\}$, by Definition 3.10 and Condition (1) of the $\bigoplus$ operator, $\Xi$ is non-empty and $\Xi \subseteq\left\{\tau_{1}, \tau_{2}\right\}$. Also, by Condition (2) of the $\bigoplus$ operator, $\mathcal{H}$ must be one of the four Herbrand sets $\{A(a), A(b)\}$, $\{B(a), B(b)\},\{A(a), B(b)\}$, and $\{B(a), A(b)\}$. That is, $\bigoplus\left\{\mathcal{F}_{1}, \mathcal{F}_{2}\right\}=\left\{\mathcal{F}_{i} \mid 1 \leq i \leq 6\right\}$ where $\mathcal{F}_{1}$ and $\mathcal{F}_{2}$ are defined as before, and

$$
\begin{array}{ll}
\mathcal{F}_{3}=\left\langle\left\{\tau_{1}, \tau_{2}\right\},\{A(a), A(b)\}\right\rangle, & \mathcal{F}_{4}=\left\langle\left\{\tau_{1}, \tau_{2}\right\},\{B(a), B(b)\}\right\rangle, \\
\mathcal{F}_{5}=\left\langle\left\{\tau_{1}, \tau_{2}\right\},\{A(a), B(b)\}\right\rangle, & \mathcal{F}_{6}=\left\langle\left\{\tau_{1}, \tau_{2}\right\},\{B(a), A(b)\}\right\rangle .
\end{array}
$$

We say a set $\mathbb{F}$ of featured interpretations is closed under $\bigoplus$ if $\bigoplus \mathbb{F}=\mathbb{F}$. It is easy to see that for any DL-Lite $\mathrm{L}_{\text {bool }}^{\mathcal{N}} \mathrm{KB} \mathcal{K}, \mathrm{FM}(\mathcal{K})$ is closed under $\bigoplus$. And being closed under $\bigoplus$ is a sufficient and necessary condition for $\mathbb{F}$ to be axiomatizable in DL-Lite $\mathrm{bool}_{\text {. }}^{\mathcal{N}}$.

PROPOSITION 3.24. For a set $\mathbb{F}$ of featured interpretations, $\mathbb{F}$ is axiomatizable in DL-Lite $e_{\text {bool }}^{\mathcal{N}}$ iff $\mathbb{F}$ is closed under $\bigoplus$.

In what follows, we slightly extend the DL-Lite language under consideration to be able to axiomatize any set of featured interpretations (not necessarily closed under $\bigoplus$ ), which includes (1) allowing disjunction of KBs and (2) using auxiliary roles to express that a concept is non-empty. Formally, a concept $C$ is non-empty in a KB $\mathcal{K}$ if $C^{\mathcal{I}} \neq \emptyset$ for each DL model $\mathcal{I}$ of $\mathcal{K}$, or equivalently (by Propositions 3.15 and 3.17), if $C$ is satisfied by each featured model $\mathcal{F}$ of $\mathcal{K}$. Note that non-emptiness is different from satisfiability as the latter requires one (featured) model to satisfy the concept.

A disjunctive knowledge base (DKB) [Meyer et al. 2005; Liu et al. 2011] is a set $\mathbb{K}$ of KBs, defined in a way that $\bmod (\mathbb{K})=\bigcup_{\mathcal{K} \in \mathbb{K}} \bmod (\mathcal{K})$. The concepts of consistency, coherence, logical entailment and equivalence of a single KB can be generalized to DKBs in a natural way. For a DKB $\mathbb{K}, F M(\mathbb{K})=\bigcup_{\mathcal{K} \in \mathbb{K}} F M(\mathcal{K})$ is the set of featured models of $\mathbb{K}$. Clearly, $F M(\mathbb{K})=\left\{\mathcal{F}_{\mathcal{I}} \mid \mathcal{I} \in\right.$ $\bmod (\mathbb{K})\}$ holds. Also, similar as the case of $\mathrm{KB}$ union, $\mathrm{FM}(\mathbb{K})=\mathrm{FM}\left(\mathcal{K}_{1}\right) \cup \mathrm{FM}\left(\mathcal{K}_{2}\right)$ for $\mathbb{K}=$ $\left\{\mathcal{K}_{1}, \mathcal{K}_{2}\right\}$. We say set $\mathbb{F}$ of featured interpretations to be DKB-axiomatizable in DL-Lite bool $^{\mathcal{N}}$ if there exists a DL-Lite $\mathrm{J}_{\text {bool }}^{\mathcal{N}}$ DKB $\mathbb{K}$ such that $\mathrm{FM}(\mathbb{K})=\mathbb{F}$.

To construct a DKB that axiomatizes $\mathbb{F}$, one additional issue is how to express in DL-Lite bool $^{\mathcal{N}}$ that a concept is non-empty (see the proof of Proposition 3.26 for details). This can be achieved by using auxiliary roles: To express that concept $D$ is non-empty whenever concept $C$ is non-empty, one can use two DL-Lite $\mathcal{N}_{\text {bool }}^{\mathcal{N}}$ TBox axioms $C \sqsubseteq \exists U$ and $\exists U^{-} \sqsubseteq D$, where $U$ is an auxiliary role name not in $\mathbb{F}$. While this does not require us to extend the DL-Lite bool $^{\mathcal{N}}$ language, a subtle issue needs to be addressed. Note that a DKB $\mathbb{K}$ containing these two axioms may have additional logical consequences about $U$ (i.e., $C \sqsubseteq \exists U$ and $\exists U^{-} \sqsubseteq D$ ) that may not follow from $\mathbb{F}$, and thus the featured models of $\mathbb{K}$ may not be exactly $\mathbb{F}$. However, the featured models of $\mathbb{K}$ would agree with $\mathbb{F}$ if $U$ is disregarded. We present this formally as follows.

We consider two signatures $\mathcal{S}$ and $\mathcal{S}^{\prime}$, where $\mathcal{S}$ is the signature for $\mathbb{F}$ (i.e., $\mathbb{F}$ is a set of $\mathcal{S}$-featured interpretations) and $\mathcal{S}^{\prime}$ extends $\mathcal{S}$ with some role names $U_{1}, \ldots, U_{n}{ }^{3}$. For an $\mathcal{S}$-type $\tau$, an $\mathcal{S}^{\prime}$-type $\tau^{\prime}$ is an $\mathcal{S}^{\prime}$-extension of $\tau$ if $\tau \subseteq \tau^{\prime}$ and $\tau^{\prime} \backslash \tau$ is empty or contains only basic concepts of the forms $\geqslant k U$ and $\geqslant k U^{-}$with $k \in \mathcal{S}_{N}$ and $U \in \mathcal{S}^{\prime} \backslash \mathcal{S}$. For a set $\Xi$ of $\mathcal{S}$-types, an $\mathcal{S}^{\prime}$-extension $\Xi^{\prime}$ of $\Xi$ consists of some $\mathcal{S}^{\prime}$-extensions of the types in $\Xi$ and at least one $\mathcal{S}^{\prime}$-extension for each type in $\Xi$. Similarly, for an $\mathcal{S}$-Herbrand set $\mathcal{H}$, an $\mathcal{S}^{\prime}$-Herbrand set $\mathcal{H}^{\prime}$ is an $\mathcal{S}^{\prime}$-extension of $\mathcal{H}$ if $\mathcal{H} \subseteq \mathcal{H}^{\prime}$ and

\footnotetext{
${ }^{3}$ The number of auxiliary roles is decided by $\mathbb{F}$, see the proof of Proposition 3.26
} 
$\mathcal{H}^{\prime} \backslash \mathcal{H}$ is empty or contains only assertions of the forms $\geqslant k U(a), \geqslant k U^{-}(a)$ and $U(a, b)$, where $k \in \mathcal{S}_{N}$ and $U \in \mathcal{S}^{\prime} \backslash \mathcal{S}$. For an $\mathcal{S}$-featured interpretation $\mathcal{F}=\langle\Xi, \mathcal{H}\rangle$, an $\mathcal{S}^{\prime}$-extension of $\mathcal{F}$ is an $\mathcal{S}^{\prime}$-featured interpretation $\left\langle\Xi^{\prime}, \mathcal{H}^{\prime}\right\rangle$, where $\Xi^{\prime}$ and $\mathcal{H}^{\prime}$ are $\mathcal{S}^{\prime}$-extensions of $\Xi$ and $\mathcal{H}$, respectively. From the definition of featured interpretations, such an $\mathcal{S}^{\prime}$-extension always exists.

For a DL-Lite $\mathcal{N}_{\text {bool }}^{\mathcal{N}} \mathrm{KB} \mathcal{K}$ over $\mathcal{S}^{\prime}$ and an $\mathcal{S}$-featured interpretation $\mathcal{F}$, we say $\mathcal{F}$ is an $\mathcal{S}$-featured model of $\mathcal{K}$ if an $\mathcal{S}^{\prime}$-extension of $\mathcal{F}$ satisfies $\mathcal{K}$. The following lemma shows that the $\mathcal{S}$-featured models of $\mathcal{K}$ faithfully characterise the semantics of $\mathcal{K}$ over $\mathcal{S}$.

LEMMA 3.25. For a $K B \mathcal{K}$ over $\mathcal{S}$ and a $K B \mathcal{K}^{\prime}$ over $\mathcal{S}^{\prime}$ such that $\mathcal{K}^{\prime} \backslash \mathcal{K}=\left\{C_{U} \sqsubseteq \exists U, \exists U^{-} \sqsubseteq\right.$ $\left.D_{U} \mid U \in \mathcal{S}^{\prime} \backslash \mathcal{S}\right\}$ where each $C_{U}$ and each $D_{U}$ are over $\mathcal{S}$, an $\mathcal{S}$-featured interpretation $\mathcal{F}$ is an $\mathcal{S}$-featured model of $\mathcal{K}^{\prime}$ iff (1) $\mathcal{F}$ is an featured model of $\mathcal{K}$ and (2) $\mathcal{F}$ satisfies concept $D_{U}$ whenever it satisfies $C_{U}$ for each $U \in \mathcal{S}^{\prime} \backslash \mathcal{S}$.

For a KB $\mathcal{K}$ over $\mathcal{S}^{\prime}$, we are mainly interested in its $\mathcal{S}$-featured models, and will use $\operatorname{FM}(\mathcal{K})$ to denote the set of $\mathcal{S}$-featured models of $\mathcal{K}$. The $\mathcal{S}$-featured models of DKBs and DKB-aximatizability are defined accordingly. We have the following expressibility result for an arbitrary set of featured interpretations.

Proposition 3.26. A set $\mathbb{F}$ of featured interpretations is always DKB-axiomatizable in DLLite $_{\text {bool }}^{\mathcal{N}}$.

When there exists no single DL-Lite ${ }_{\text {bool }}^{\mathcal{N}}$ KB over $\mathcal{S}$ that axiomatizes the given set $\mathbb{F}$ of featured interpretations, we can use a DL-Lite bool $_{\mathcal{N}}^{\mathcal{N}} \mathrm{KB}$ over $\mathcal{S}$ to approximate $\mathbb{F}$. As the MA of a set of interpretations, a maximal approximation (MA) of a set $\mathbb{F}$ of featured interpretations over $\mathcal{S}$ in DLLite $_{\text {bool }}^{\mathcal{N}}$ is a DL-Lite $\mathcal{L}_{\text {bool }}^{\mathcal{N}} \mathrm{KB} \mathcal{K}$ such that: (1) $\operatorname{sig}(\mathcal{K}) \subseteq \mathcal{S}$ and $\mathbb{F} \subseteq \mathrm{FM}(\mathcal{K})$, and (2) there exists no DL-Lite $\mathcal{L}_{\text {bool }}^{\mathcal{N}} \mathrm{KB} \mathcal{K}^{\prime}$ satisfying (1) and $\operatorname{FM}\left(\mathcal{K}^{\prime}\right) \subset \mathrm{FM}(\mathcal{K})$. For a set $\mathbb{F}$ of featured interpretations, the MA of $\mathbb{F}$ is unique up to KB equivalence. This can be seen as follows: Suppose both $\mathcal{K}$ and $\mathcal{K}^{\prime}$ are MAs of $\mathbb{F}$, then by Proposition 3.18 $\mathcal{K} \cup \mathcal{K}^{\prime}$ is a MA of $\mathbb{F}$, which implies that $\mathcal{K} \equiv \mathcal{K}^{\prime}$. Moreover, as a direct conclusion from Proposition 3.24 , the following corollary holds.

Corollary 3.27. Let $\mathcal{K}$ be the MA of $\mathbb{F}$ in DL-Lite ${ }_{\text {bool }}^{\mathcal{N}}$. Then, $\operatorname{FM}(\mathcal{K})=\bigoplus \mathbb{F}$.

LEMMA 3.28. Given a set $\mathbb{M}$ of interpretations and a signature $\mathcal{S}$, let $\mathbb{F}=\left\{\mathcal{F}_{\mathcal{I}} \mid \mathcal{I} \in \mathbb{M}\right\}$. The MA of $\mathbb{F}$ in DL-Lite $\mathcal{b}_{\text {bool }}^{\mathcal{N}}$ is exactly the MA of $\mathbb{M}$ over $\mathcal{S}$ in DL-Lite bool $^{\mathcal{N}}$.

In the next section, we will introduce two definitions of distance between featured interpretations. Once a notion of distances between featured interpretations is provided, we can easily define an operator of DL-Lite revision.

\section{TWO REVISION OPERATORS}

In this section, based on the semantic characterisation introduced previously, we will define two different operators for ontology revision and investigate their properties including AGM postulates. Especially, the rationality of our revision operators is justified by AGM postulates. An application of our revision operators is demonstrated through a practical example of ontology revision adapted from NCI Thesaurus.

Ontology revision is the task of revising an existing ontology (i.e., a DL KB) $\mathcal{K}$ by a set of new TBox and ABox axioms (i.e., a new $\mathrm{KB} \mathcal{K}^{\prime}$ ). Usually, $\mathcal{K}^{\prime}$ is considered to be more up-todate and more accurate than $\mathcal{K}$. As a result, $\mathcal{K}^{\prime}$ is fully preserved during the revision, and if there is any inconsistency, $\mathcal{K}$ can be modified. As the initial knowledge $\mathcal{K}$ is also of great value, the revision should make as little change to $\mathcal{K}$ as possible. This criteria is well known as the principle of minimal change in belief revision and ontology revision, and it has been formulated in several different ways. Classical model-based revision approaches achieve minimal change by measuring distances between propositional models, where a propositional model is a set of atoms. As explained before, ontology revision is more challenging than classical belief revision in that we need to handle (essentially) first order KBs instead of propositional ones. 
We define our revision operators by adapting a classical model-based revision approach but using the syntactic structure of featured models instead of DL models. Several different notions of distance have been proposed in classical belief revision. Among them, Dalal's distance [Dalal 1988] and Satoh's distances [Satoh 1988] are the two most prevailing ones. Further details of such revision operators can be found in [Katsuno and Mendelzon 1991: Eiter and Gottlob 1992]. Informally, both Dalal's and Satoh's distances aim to characterise the difference of atoms in two propositional models. However, these two distances differ in that Dalal defines the distance to be the cardinality of the symmetric difference of two propositional models, whereas Satoh defines the distance to be exactly the symmetric difference set. Thus, Satoh's distance is more fine-grained. In practical ontology applications, it is highly relevant to know which predicates/atoms are interpreted differently in two models, instead of simply their numbers. Hence, we adopt Satoh's notion of distance in our approach, while we remark that our results can be mathematically adapted to Dalal's distance.

We propose two notions of distance between featured models, in the same spirit of Satoh's. The first distance is defined as the set of concepts and roles interpreted differently in the two featured models, and the second distance is based on a generalized notion of symmetric difference. We show that the first distance reflects differences on predicate level between classical DL interpretations, while the second distance is more fine-grained and reveals more subtle differences. Based on these two distances, we define two specific revision operators for DL-Lite KBs in an analogous way to Satoh's, in a model-theoretical manner using featured models and distances.

\subsection{Predicate Difference and P-Revision}

Predicates, i.e., concept names and role names, are the basic elements in the definition of a classical DL interpretation, and hence the authors of [Qi and Du 2009] define a distance between two DL interpretations as a set of predicates on which the two interpretations disagree. Our first notion of distance between featured models is in the same spirit, and is defined through the (minimal sets of) predicates that are interpreted differently by two featured models.

Let $\Sigma_{\mathcal{S}}=\mathcal{S}_{C} \cup \mathcal{S}_{R}$ be the set of concept names and role names on the signature $\mathcal{S}$, and $\tau_{1}$ and $\tau_{2}$ be two $\mathcal{S}$-types. For a subset $\Sigma$ of $\Sigma_{\mathcal{S}}$, by $\tau_{1} \sim_{\Sigma} \tau_{2}$ we mean that $\tau_{1}$ and $\tau_{2}$ agree on all predicates in $\Sigma$, that is, for every basic concept $B$ over $\Sigma, B \in \tau_{1}$ iff $B \in \tau_{2}$. Intuitively, if $\tau_{1} \sim_{\Sigma} \tau_{2}$, then $\tau_{1}$ and $\tau_{2}$ have the same interpretation on $\Sigma$. This relation on types can be extended to featured interpretations as follows. Let $\mathcal{F}_{1}=\left\langle\Xi_{1}, \mathcal{H}_{1}\right\rangle$ and $\mathcal{F}_{2}=\left\langle\Xi_{2}, \mathcal{H}_{2}\right\rangle$ be two featured interpretations over $\mathcal{S}$. Define $\mathcal{F}_{1} \sim_{\Sigma} \mathcal{F}_{2}$ if the following two conditions are satisfied:

(1) For each $\tau_{1} \in \Xi_{1}$, there exists $\tau_{2} \in \Xi_{2}$ s.t. $\tau_{1} \sim_{\Sigma} \tau_{2}$; conversely, for each $\tau_{2} \in \Xi_{2}$, there exists $\tau_{1} \in \Xi_{1}$ s.t. $\tau_{1} \sim_{\Sigma} \tau_{2}$.

(2) For each $a \in \mathcal{S}_{I}, \tau^{\mathcal{H}_{1}}(a) \sim_{\Sigma} \tau^{\mathcal{H}_{2}}(a)$; and for each $P \in \mathcal{S}_{R} \cap \Sigma$ and $a, b \in \mathcal{S}_{I}, P(a, b) \in \mathcal{H}_{1}$ iff $P(a, b) \in \mathcal{H}_{2}$.

For example, given types $\tau_{1}=\{A\}, \tau_{2}=\{A, B, C\}, \tau_{3}=\{A, B\}, \tau_{4}=\{A, C\}$, and featured models $\mathcal{F}_{1}=\left\langle\left\{\tau_{1}, \tau_{2}\right\},\{A(a)\}\right\rangle, \mathcal{F}_{2}=\left\langle\left\{\tau_{3}, \tau_{4}\right\},\{A(a), B(a)\}\right\rangle$, then $\mathcal{F}_{1} \sim_{\{A\}} \mathcal{F}_{2}$ and $\mathcal{F}_{1} \sim_{\{A, C\}} \mathcal{F}_{2}$.

To define the distance between two featured interpretations, we are interested in the minimal sets of predicates (concepts and roles) that are interpreted differently by these two featured interpretations. Note that there can be more than one such minimal sets. For example, let $\mathcal{F}_{3}=\left\langle\left\{\tau_{1}, \tau_{3}, \tau_{4}\right\},\{A(a)\}\right\rangle$, where $\tau_{1}, \tau_{2}, \tau_{3}$ and $\mathcal{F}_{1}$ are as above, then $\mathcal{F}_{1} \sim_{\{A, B\}} \mathcal{F}_{3}$ and $\mathcal{F}_{1} \sim\{A, C\} \mathcal{F}_{3}$. That is, the minimal sets on which $\mathcal{F}_{1}$ and $\mathcal{F}_{3}$ disagree are $\{C\}$ and $\{B\}$. For two featured interpretations $\mathcal{F}_{1}$ and $\mathcal{F}_{2}$ over $\mathcal{S}$, we define the $P$-distance between $\mathcal{F}_{1}$ and $\mathcal{F}_{2}$, denoted $d_{P}\left(\mathcal{F}_{1}, \mathcal{F}_{2}\right)$, to be the set of all minimal sets $\Sigma$ such that $\mathcal{F}_{1} \sim_{\bar{\Sigma}} \mathcal{F}_{2}$ where $\bar{\Sigma}=\Sigma_{\mathcal{S}} \backslash \Sigma$. In the above example, $d_{P}\left(\mathcal{F}_{1}, \mathcal{F}_{2}\right)=\{\{B\}\}$ and $d_{P}\left(\mathcal{F}_{1}, \mathcal{F}_{3}\right)=\{\{B\},\{C\}\}$. 
For two KBs $\mathcal{K}_{1}, \mathcal{K}_{2}$ and $\mathcal{S}=\operatorname{sig}\left(\mathcal{K}_{1} \cup \mathcal{K}_{2}\right), \operatorname{MD}_{P}\left(\mathcal{K}_{1}, \mathcal{K}_{2}\right)$ consists of all minimal sets $\Sigma$ occurring in the P-distances between the featured models of $\mathcal{K}_{1}$ and $\mathcal{K}_{2}$ :

$$
\operatorname{MD}_{P}\left(\mathcal{K}_{1}, \mathcal{K}_{2}\right)=\min _{\subseteq}\left(\bigcup_{\mathcal{F}_{1} \in \operatorname{FM}\left(\mathcal{K}_{1}\right), \mathcal{F}_{2} \in \operatorname{FM}\left(\mathcal{K}_{2}\right)} d_{P}\left(\mathcal{F}_{1}, \mathcal{F}_{2}\right)\right) .
$$

Intuitively, $\operatorname{MD}_{P}\left(\mathcal{K}_{1}, \mathcal{K}_{2}\right)$ collects all the minimal sets of predicates that are interpreted differently between the featured models of $\mathcal{K}_{1}$ and $\mathcal{K}_{2}$. For a pair of featured models $\mathcal{F}_{1}$ and $\mathcal{F}_{2}$ of $\mathcal{K}_{1}$ and $\mathcal{K}_{2}$, respectively, we say the $\mathrm{P}$-distance between $\mathcal{F}_{1}$ and $\mathcal{F}_{2}$ is minimal between $\mathcal{K}_{1}$ and $\mathcal{K}_{2}$ if it contains some $\Sigma \in \operatorname{MD}_{P}\left(\mathcal{K}_{1}, \mathcal{K}_{2}\right)$. When $\mathcal{K}_{1}$ and $\mathcal{K}_{2}$ are clear from the context, we will also say the P-distance is minimal.

To define a revision operator $\mathcal{K} \circ \mathcal{K}^{\prime}$ in analogy to classical model-based revision, it amounts to specify the set of featured models of $\mathcal{K}^{\prime}$ that is closest to $\mathcal{K}$ with respect to P-distances. We say a featured model $\mathcal{F}^{\prime}$ of $\mathcal{K}^{\prime}$ is $P$-closest to $\mathrm{KB} \mathcal{K}$ if there exists a featured model $\mathcal{F}$ of $\mathcal{K}$ such that the P-distance between $\mathcal{F}$ and $\mathcal{F}^{\prime}$ is minimal between $\mathcal{K}$ and $\mathcal{K}^{\prime}$.

Now we are ready to present our first revision operator.

Definition 4.1 (P-Revision). Let $\mathcal{K}, \mathcal{K}^{\prime}$ be two $\mathrm{KBs}$ and $\mathcal{S}=\operatorname{sig}\left(\mathcal{K} \cup \mathcal{K}^{\prime}\right)$. The predicate difference-based revision (P-revision) of $\mathcal{K}$ by $\mathcal{K}^{\prime}$ is a $\mathrm{DKB} \mathcal{K} \circ_{P} \mathcal{K}^{\prime}$, such that $\mathrm{FM}\left(\mathcal{K} \circ_{P} \mathcal{K}^{\prime}\right)=$ $\operatorname{FM}\left(\mathcal{K}^{\prime}\right)$ if $\operatorname{FM}(\mathcal{K})=\emptyset$, and otherwise, $\operatorname{FM}\left(\mathcal{K} \circ_{P} \mathcal{K}^{\prime}\right)$ is the set of all featured models in $\operatorname{FM}\left(\mathcal{K}^{\prime}\right)$ that are P-closest to $\mathcal{K}$.

P-revision is well defined as $\operatorname{FM}\left(\mathcal{K} \circ_{P} \mathcal{K}^{\prime}\right)$ is always DKB-axiomatizable in DL-Lite $\mathcal{N}_{\text {bool }}$ by Proposition 3.26 .

Example 4.2. Consider the following $\mathrm{KB}$,

$$
\begin{aligned}
\mathcal{K}=\langle & \{\text { PhDStudent } \sqsubseteq \text { Student, Student } \sqsubseteq \neg \exists \text { hasStaffID, } \\
& \left.\left.\exists \text { hasStaffID }{ }^{-} \sqsubseteq \mathrm{ID}, \text { Student } \sqcap \mathrm{ID} \sqsubseteq \perp\right\},\{\text { PhDStudent(John) }\}\right\rangle .
\end{aligned}
$$

The TBox of $\mathcal{K}$ specifies that PhD students are students, and students are not allowed to have staff IDs, while the ABox states that John is a PhD student. Suppose PhD students do have staff IDs, and John has staff ID S0564. We want to revise $\mathcal{K}$ with

$$
\mathcal{K}^{\prime}=\langle\{\text { PhDStudent } \sqsubseteq \exists \text { hasStaffID }\},\{\text { hasStaffID(John, S0564) }\}\rangle .
$$

Take

$$
\begin{aligned}
& \left.\mathcal{F}=\left\langle\left\{\tau_{1}, \tau_{2}\right\},\{\operatorname{PhDStudent}(\text { John }) \text {, Student (John })\right\}\right\rangle \text { and } \\
& \mathcal{F}^{\prime}=\left\langle\left\{\tau_{3}, \tau_{4}\right\},\{\text { PhDStudent(John), Student(John), hasStaffID(John, S0564), }\right. \\
& \exists \text { hasStaffID(John), } \exists \text { hasStaffID }{ }^{-}(\text {S0564)\} }\rangle
\end{aligned}
$$

where $\tau_{1}=\{$ PhDStudent, Student $\}, \tau_{2}=\emptyset, \tau_{3}=\{$ PhDStudent, Student, $\exists$ hasStaffID $\}$, and $\tau_{4}=\left\{\exists\right.$ hasStaffID $\left.{ }^{-}\right\}$. Then $\mathcal{F}$ and $\mathcal{F}^{\prime}$ are featured models of $\mathcal{K}$ and $\mathcal{K}^{\prime}$, respectively. Moreover, $d_{P}\left(\mathcal{F}, \mathcal{F}^{\prime}\right)=\left\{\{\right.$ hasStaffID $\}$ and $\{$ hasStaffID $\}$ is in $\operatorname{MD}_{P}\left(\mathcal{K}, \mathcal{K}^{\prime}\right)$. That is, $\mathcal{F}^{\prime}$ is P-closest to $\mathcal{K}$ and thus it is a featured model of $\mathcal{K} \circ_{P} \mathcal{K}^{\prime}$. Indeed, $\{$ hasStaffID $\}$ is the only predicate set in $\operatorname{MD}_{P}\left(\mathcal{K}, \mathcal{K}^{\prime}\right)$, and $\operatorname{FM}\left(\mathcal{K} \circ_{P} \mathcal{K}^{\prime}\right)$ consists of those featured models of $\mathcal{K}^{\prime}$ that (possibly) disagree with some featured models of $\mathcal{K}$ only on hasStaffID.

In fact, the P-revision of $\mathcal{K}$ by $\mathcal{K}^{\prime}$ can be expressed as a single DL-Lite $\mathcal{b}_{\text {bool }}^{\mathcal{N}} \mathrm{KB}$ :

$$
\begin{aligned}
\mathcal{K} \circ_{P} \mathcal{K}^{\prime}=\langle & \{\text { PhDStudent } \sqsubseteq \text { Student, Student } \sqcap \text { ID } \sqsubseteq \perp, \text { PhDStudent } \sqsubseteq \exists \text { hasStaffID }\}, \\
& \{\text { PhDStudent (John), hasStaffID(John, S0564) }\}\rangle .
\end{aligned}
$$

The result of P-revision can be obtained in terms of forgetting, an operator that eliminates unnecessary predicates from a given KB [Wang et al. 2010c]. In Example 4.2, initial knowledge in $\mathcal{K}$ about role hasStaffID is forgotten to incorporate the new knowledge $\mathcal{K}^{\prime}$. 
The relevant notion of forgetting in [Wang et al. 2010c] is defined on a slight extension of DLLite $_{\text {bool }}^{\mathcal{N}}$, called DL-Lite ${ }_{\text {bool }}^{u}$, which allows concepts of the form $\exists u . C$ with $u$ being a special universal role (analogous to $T$ for concepts)

Definition 4.3. [Wang et al. 2010c] Let $\mathcal{K}$ be a KB, $\Sigma \subseteq \Sigma_{\mathcal{S}}$ be a set of predicates. Then, KB $\mathcal{K}^{\prime}$ is a result of $u$-forgetting about $\Sigma$ in $\mathcal{K}$ if

$-\operatorname{sig}\left(\mathcal{K}^{\prime}\right) \subseteq \operatorname{sig}(\mathcal{K}) \backslash \Sigma$;

$-\mathcal{K} \models \mathcal{K}^{\prime}$

$-\mathcal{K} \models C \sqsubseteq D$ iff $\mathcal{K}^{\prime} \models C \sqsubseteq D$, for each pair of DL-Lite bool concepts $C$ and $D$ with $\operatorname{sig}(C \sqsubseteq$ $D) \cap \Sigma=\emptyset$;

$-\mathcal{K} \models C(a)$ iff $\mathcal{K}^{\prime} \models C(a)$, for each DL-Lite bool concept $C$ with $\operatorname{sig}(C) \cap \Sigma=\emptyset$.

It is shown in [Wang et al. 2010c] that such a $\mathrm{KB} \mathcal{K}^{\prime}$, denoted forget $(\mathcal{K}, \Sigma)$, can be constructed in DL-Lite $_{\text {bool }}^{u}$, and is unique up to KB equivalence. Without loss of generality, we can assume that the special role $u$ occurs in forget $(\mathcal{K}, \Sigma)$ only in axioms of the form $C \sqsubseteq \exists u$. $D$ where $C$ and $D$ are DL-Lite $\mathcal{N}_{\text {bool }}^{\mathcal{N}}$ concepts. The semantics of $C \sqsubseteq \exists u . D$ can be well captured by two axioms $C \sqsubseteq \exists U$ and $\exists U^{-} \sqsubseteq D$ with an auxiliary role $U$ (note that auxiliary roles are not allowed in the forgetting operation and hence the extension DL-Lite ${ }_{\text {bool }}^{u}$ is necessary). Let forget ${ }^{U}(\mathcal{K}, \Sigma)$ denotes the DLLite $_{\text {bool }}^{\mathcal{N}} \mathrm{KB}$ obtained from forget $(\mathcal{K}, \Sigma)$ by replacing each axiom $C \sqsubseteq \exists u . D$ with two axioms $C \sqsubseteq \exists U_{C, D}$ and $\exists U_{C, D}^{-} \sqsubseteq D$ where each $U_{C, D}$ is a distinct fresh role name.

The following result connects P-revision to forgetting.

Proposition 4.4. Let $\mathcal{K}, \mathcal{K}^{\prime}$ be two consistent $D L-L i t e_{\text {bool }}^{\mathcal{N}} K B s$ and $\operatorname{sig}\left(\mathcal{K} \cup \mathcal{K}^{\prime}\right) \subseteq \mathcal{S}$. Then,

$$
\mathcal{K} \circ_{P} \mathcal{K}^{\prime}=\left\{\operatorname{forget}^{U}(\mathcal{K}, \Sigma) \cup \mathcal{K}^{\prime} \mid \Sigma \in \operatorname{MD}_{P}\left(\mathcal{K}, \mathcal{K}^{\prime}\right)\right\}
$$

Note that $\mathcal{K} \circ_{P} \mathcal{K}^{\prime}$ in the above proposition is a DKB.

We remark that the result of P-revision may not be expressible as a single KB. This can be seen from the following example.

Example 4.5. Let $\mathcal{K}=\langle\emptyset,\{P(a, b), R(a, b)\}\rangle$ where $P$ and $R$ are two distinct role names, and $\mathcal{K}^{\prime}=\langle\{\exists P \sqcap \exists R \sqsubseteq \perp\}, \emptyset\rangle$ stating that $P$ and $R$ must have disjoint domains. Then, the result of revising $\mathcal{K}$ by $\mathcal{K}^{\prime}$ is a DKB $\left\{\mathcal{K}_{1}, \mathcal{K}_{2}\right\}$ with each of $\mathcal{K}_{i}(i=1,2)$ containing either $P(a, b)$ or $R(a, b)$, but not both. Since one cannot express disjunctive role assertions in DL-Lite, the result of revision cannot be expressed as a single KB.

For applications where it is desirable to have the result of revision as a single DL-Lite $\mathcal{N}_{\text {bool }}^{\mathcal{N}} \mathrm{KB}$ rather than a DKB, the maximal approximation (MA) of revision is used as the desired result. The MA of P-revision, $\mathrm{MA}\left(\mathcal{K} \circ_{P} \mathcal{K}^{\prime}\right)$, is the MA of $\operatorname{FM}\left(\mathcal{K} \circ_{P} \mathcal{K}^{\prime}\right)$ in DL-Lite ${ }_{\text {bool }}^{\mathcal{N}}$. In Example 4.5 . $\operatorname{MA}\left(\mathcal{K} \circ_{P} \mathcal{K}^{\prime}\right)$ is $\mathcal{K}^{\prime}$.

In what follows, we show that our definition of P-distance based on featured models faithfully reflects differences in interpretation of predicates in classical DL models. In particular, we show that P-revision captures the revision results defined analogously using classical DL models.

For two DL interpretations $\mathcal{I}_{1}$ and $\mathcal{I}_{2}$, define the $P^{*}$-distance between $\mathcal{I}_{1}$ and $\mathcal{I}_{2}$ to be

$$
d_{P^{*}}\left(\mathcal{I}_{1}, \mathcal{I}_{2}\right)=\left\{E \in \Sigma_{\mathcal{S}} \mid E^{\mathcal{I}_{1}} \neq E^{\mathcal{I}_{2}}\right\}
$$

Intuitively, $d_{P^{*}}\left(\mathcal{I}_{1}, \mathcal{I}_{2}\right)$ is the set of predicates on which $\mathcal{I}_{1}$ and $\mathcal{I}_{2}$ interpret differently. Note that $d_{P^{*}}\left(\mathcal{I}_{1}, \mathcal{I}_{2}\right)$ generalises the difference set in (Definition 1 of) [Qi and Du 2009] which consists of only concept names. For two KBs $\mathcal{K}_{1}, \mathcal{K}_{2}$ and $\mathcal{S}=\operatorname{sig}\left(\mathcal{K}_{1} \cup \mathcal{K}_{2}\right), \operatorname{MD}_{P^{*}}\left(\mathcal{K}_{1}, \mathcal{K}_{2}\right)$ is the set of all minimal $\mathrm{P}^{*}$-distances between the DL models of $\mathcal{K}_{1}$ and $\mathcal{K}_{2}$ :

$$
\operatorname{MD}_{P^{*}}\left(\mathcal{K}_{1}, \mathcal{K}_{2}\right)=\min _{\subseteq}\left(\left\{d_{P^{*}}\left(\mathcal{I}_{1}, \mathcal{I}_{2}\right) \mid \mathcal{I}_{1} \in \bmod \left(\mathcal{K}_{1}\right) \text { and } \mathcal{I}_{2} \in \bmod \left(\mathcal{K}_{2}\right)\right\}\right)
$$

\footnotetext{
${ }^{4}$ The reader should refer to Kontchakov et al. 2010. Wang et al. 2010c for details of DL-Lite ${ }_{\text {bool }}^{u}$.
} 
We say a DL model $\mathcal{I}_{2}$ of $\mathcal{K}_{2}$ is $P^{*}$-closest to $\mathcal{K}_{1}$ if there exists a DL model $\mathcal{I}_{1}$ of $\mathcal{K}_{1}$ such that $d_{P^{*}}\left(\mathcal{I}_{1}, \mathcal{I}_{2}\right) \in \operatorname{MD}_{P^{*}}\left(\mathcal{K}_{1}, \mathcal{K}_{2}\right)$.

The following definition is analogous to P-revision but is defined using DL models.

Definition $4.6\left(P^{*}\right.$-Revision $)$. Let $\mathcal{K}, \mathcal{K}^{\prime}$ be two $\mathrm{KBs}$ and $\mathcal{S}=\operatorname{sig}\left(\mathcal{K} \cup \mathcal{K}^{\prime}\right)$. The $P^{*}$-revision of $\mathcal{K}$ by $\mathcal{K}^{\prime}$, denoted $\mathcal{K} \circ_{P^{*}} \mathcal{K}^{\prime}$, is defined by its models: $\bmod \left(\mathcal{K} \circ_{P^{*}} \mathcal{K}^{\prime}\right)=\bmod \left(\mathcal{K}^{\prime}\right)$ if $\bmod (\mathcal{K})=\emptyset$, and otherwise, $\bmod \left(\mathcal{K} \circ_{P^{*}} \mathcal{K}^{\prime}\right)$ is the set of all DL models in $\bmod \left(\mathcal{K}^{\prime}\right)$ that are $\mathrm{P}^{*}$-closest to $\mathcal{K}$.

Unlike P-revision, a result of $\mathrm{P}^{*}$-revision may not be DKB-axiomatizable in DL-Lite $\mathcal{L}_{\text {bool }}^{\mathcal{N}}$, that is, there may not exist a DKB in DL-Lite ${ }_{\text {bool }}^{\mathcal{N}}$ whose DL models are exactly $\bmod \left(\mathcal{K} o_{P^{*}} \mathcal{K}^{\prime}\right)$. Indeed, it is an open question in which extension of DL-Lite $\mathrm{P}^{*}$-revision is axiomatizable. Also, even if a result of $\mathrm{P}^{*}$-revision is axiomatizable, it is unclear how to compute it. Before presenting an example to show the inexpressibility, we first establish a useful connection between $\mathrm{P}^{*}$-revision and $\mathrm{P}$-revision. In particular, it says the featured models of P-revision are exactly those induced by the DL models of $\mathrm{P} *$-revision.

\section{Proposition 4.7. For two KBs $\mathcal{K}$ and $\mathcal{K}^{\prime}, \operatorname{FM}\left(\mathcal{K} \circ_{P} \mathcal{K}^{\prime}\right)=\left\{\mathcal{F}_{\mathcal{I}} \mid \mathcal{I} \in \bmod \left(\mathcal{K} o_{P^{*}} \mathcal{K}^{\prime}\right)\right\}$}

From Proposition 3.17 and Theorem 3.21 it is easy to see the following corollary holds, which essentially connects our revision with model-based revision. As with $\mathrm{P}$-revision, $\mathrm{MA}\left(\mathcal{K} \circ_{P^{*}} \mathcal{K}^{\prime}\right)$ is the MA of $\bmod \left(\mathcal{K} \circ_{P^{*}} \mathcal{K}^{\prime}\right)$ in DL-Lite $\mathcal{b o o l}^{\mathcal{N}}$.

Corollary 4.8. $\operatorname{MA}\left(\mathcal{K} \circ_{P^{*}} \mathcal{K}^{\prime}\right) \equiv \operatorname{MA}\left(\mathcal{K} \circ_{P} \mathcal{K}^{\prime}\right)$; and $\mathcal{K} \circ_{P^{*}} \mathcal{K}^{\prime} \equiv \mathcal{K} o_{P} \mathcal{K}^{\prime}$ whenever $\mathcal{K} \circ_{P^{*}} \mathcal{K}^{\prime}$ is DKB-axiomatizable in DL-Lite bool $^{\mathcal{N}}$

Now, we present an example to show that a result of $\mathrm{P}^{*}$-revision may not be DKB-axiomatizable in DL-Lite $\mathcal{N}_{\text {bool }}^{\mathcal{N}}$.

Example 4.9 (Cont. Example 2.1). Consider the $\mathrm{KB} \mathcal{K}$ in Example 2.1 and a new $\mathrm{KB} \mathcal{K}^{\prime}=$ $\langle\emptyset,\{P(b, b)\}\rangle$. Adding $\mathcal{K}^{\prime}$ to $\mathcal{K}$ introduces inconsistency, as $P(b, b)$ contradicts the assertions $P(a, b)$ and $\geqslant 2 P^{-} \sqsubseteq \perp$ in $\mathcal{K}$. From the definition of P-revision, there is a single minimal set $\{P\}$ in $\operatorname{MD}_{P}\left(\mathcal{K}, \mathcal{K}^{\prime}\right)$, and we can compute through forgetting that $\mathcal{K} \circ_{P} \mathcal{K}^{\prime}=\langle\{A \sqcap B \sqsubseteq$ $\perp\},\{A(a), B(b), P(b, b)\}\rangle$.

Suppose $\mathcal{K} \circ_{P^{*}} \mathcal{K}^{\prime}$ is expressible as a DKB in DL-Lite ${ }_{\text {bool }}^{\mathcal{N}}$, then by Corollary $4.8, \mathcal{K} \circ_{P^{*}} \mathcal{K}^{\prime} \equiv$ $\mathcal{K} \circ_{P} \mathcal{K}^{\prime}$. Also, from the definition of $\mathrm{P}^{*}$-revision, for each DL model $\mathcal{I}$ of $\mathcal{K} \circ_{P^{*}} \mathcal{K}^{\prime}$ there should be a DL model $\mathcal{I}^{\prime}$ of $\mathcal{K}$ with $d_{P^{*}}\left(\mathcal{I}, \mathcal{I}^{\prime}\right)=\{P\}$. However, it is not the case. Take a model $\mathcal{I}$ of $\mathcal{K} \circ_{P^{*}} \mathcal{K}^{\prime}$ with $\Delta^{\mathcal{I}}=\{a, b\}, A^{\mathcal{I}}=\{a\}, B^{\mathcal{I}}=\{b\}$, and $P^{\mathcal{I}}=\{(b, b)\}$. There does not exist a model $\mathcal{I}^{\prime}$ of $\mathcal{K}$ satisfying the above condition, since in each model $\mathcal{I}^{\prime}$ of $\mathcal{K}, B^{\mathcal{I}^{\prime}}$ must be an infinite set (see Example 2.1). Hence, $B^{\mathcal{I}} \neq B^{\mathcal{I}^{\prime}}$, which contradicts $d_{P^{*}}\left(\mathcal{I}, \mathcal{I}^{\prime}\right)=\{P\}$. Thus, $\mathcal{K} \circ_{P^{*}} \mathcal{K}^{\prime}$ is not expressible as a DKB in DL-Lite $\mathcal{L}_{\text {bool }}^{\mathcal{N}}$.

Corollary 4.8 suggests that P-revision is a good approximation of $\mathrm{P} *$-revision: they have the same MA; P-revision is always DKB-axiomatizable in DL-Lite ${ }_{\text {bool }}^{\mathcal{N}}$ whereas $\mathrm{P}^{*}$-revision is not, and $\mathrm{P}$-revision coincides with $\mathrm{P}^{*}$-revision whenever the latter is also axiomatizable. Moreover, it is unknown how to directly compute the MA of $\mathrm{P}^{*}$-revision in DL-Lite ${ }_{\text {bool }}^{\mathcal{N}}$, and we show in Section 5 an algorithm to compute it via the MA P-revision.

\subsection{Symmetric Difference and S-Revision}

While the definition of P-revision is simple and intuitive, some applications may require the revision result to preserve more initial knowledge. Especially, in Example 4.2, all the knowledge about role hasStaffID from $\mathcal{K}$ is lost during revision. Such a behaviour of $\mathrm{P}$-revision can be explained through its connection with forgetting $-\{$ hasStaffID $\}$ occurs in $\operatorname{MD}_{P}\left(\mathcal{K}, \mathcal{K}^{\prime}\right)$ and thus the role is forgotten during revision. Indeed, the P-distance defined via predicates is sometimes insufficient to reflect subtle differences between models, as shown by the following example. Consider types $\tau_{1}=\{A\}$, $\tau_{2}=\{B\}, \tau_{3}=\emptyset$, and featured interpretations 


$$
\begin{aligned}
\mathcal{F} & =\left\langle\left\{\tau_{1}, \tau_{2}\right\},\{A(a), B(b), A(c), A(d)\}\right\rangle, \\
\mathcal{F}^{\prime} & =\left\langle\left\{\tau_{1}, \tau_{2}\right\},\{A(a), A(b), A(c), A(d)\}\right\rangle, \text { and } \\
\mathcal{F}^{\prime \prime} & =\left\langle\left\{\tau_{1}, \tau_{3}\right\},\{A(a), A(b)\}\right\rangle .
\end{aligned}
$$

Intuitively, $\mathcal{F}$ is closer to $\mathcal{F}^{\prime}$ than to $\mathcal{F}^{\prime \prime}$. Yet such a difference cannot be reflected by the P-distance, as $d_{P}\left(\mathcal{F}, \mathcal{F}^{\prime}\right)=d_{P}\left(\mathcal{F}, \mathcal{F}^{\prime \prime}\right)=\{\{A, B\}\}$. Thus, the P-revision operator is blinded from subtle differences between featured models and hence fails to eliminate less desired candidates like $\mathcal{F}^{\prime \prime}$.

The above observation motivates us to look into a more fine-grained way to adapt Satoh's distance to featured models. The new distance between two featured models is based on the symmetric difference $\triangle$ of two sets. Recall that $S_{1} \triangle S_{2}=\left(S_{1} \backslash S_{2}\right) \cup\left(S_{2} \backslash S_{1}\right)$ for two sets $S_{1}$ and $S_{2}$.

For two featured interpretations $\mathcal{F}_{1}=\left\langle\Xi_{1}, \mathcal{H}_{1}\right\rangle$ and $\mathcal{F}_{2}=\left\langle\Xi_{2}, \mathcal{H}_{2}\right\rangle$ over $\mathcal{S}$, the $S$ distance between $\mathcal{F}_{1}$ and $\mathcal{F}_{2}$ is $d_{S}\left(\mathcal{F}_{1}, \mathcal{F}_{2}\right)=\left\langle\Xi_{1} \triangle \Xi_{2}, \mathcal{H}_{1} \triangle \mathcal{H}_{2}\right\rangle$. Clearly, S-distances are more informative than P-distances. In the above example, $d_{S}\left(\mathcal{F}, \mathcal{F}^{\prime}\right)=\langle\emptyset,\{A(b), B(b)\}\rangle$ and $d_{S}\left(\mathcal{F}, \mathcal{F}^{\prime \prime}\right)=\left\langle\left\{\tau_{2}, \tau_{3}\right\},\{A(b), B(b), A(c), A(d)\}\right\rangle$. Note that we do not require $d_{S}\left(\mathcal{F}_{1}, \mathcal{F}_{2}\right)$ to be a featured interpretation. For example, let $\mathcal{S}=\left\{P, a, b_{1}, b_{2}, b_{3}, 1,2,3\right\}, \mathcal{H}_{1}=$ $\left\{\exists P(a), P\left(a, b_{1}\right)\right\}$ and $\mathcal{H}_{2}=\left\{\exists P(a),(\geqslant 2 P)(a), P\left(a, b_{2}\right), P\left(a, b_{3}\right)\right\}$. Then, $\mathcal{H}_{1} \triangle \mathcal{H}_{2}=\{(\geqslant$ $\left.2 P)(a), P\left(a, b_{1}\right), P\left(a, b_{2}\right), P\left(a, b_{3}\right)\right\}$, which is not a Herbrand set because it does not contain $\exists P(a)$ (Condition (1)of Definition 3.7) and $(\geqslant 3 P)(a)$ (Condition (2) of Definition 3.7).

To compare two S-distances, we can compare both components in S-distances and define $d_{S}\left(\mathcal{F}_{1}, \mathcal{F}_{2}\right) \subseteq d_{S}\left(\mathcal{F}_{3}, \mathcal{F}_{4}\right)$ if $\Xi_{1} \triangle \Xi_{2} \subseteq \Xi_{3} \triangle \Xi_{4}$ and $\mathcal{H}_{1} \triangle \mathcal{H}_{2} \subseteq \mathcal{H}_{3} \triangle \mathcal{H}_{4}$; and $d_{S}\left(\mathcal{F}_{1}, \mathcal{F}_{2}\right) \subset$ $d_{S}\left(\mathcal{F}_{3}, \mathcal{F}_{4}\right)$ if $d_{S}\left(\mathcal{F}_{1}, \mathcal{F}_{2}\right) \subseteq d_{S}\left(\mathcal{F}_{3}, \mathcal{F}_{4}\right)$ and $d_{S}\left(\mathcal{F}_{3}, \mathcal{F}_{4}\right) \nsubseteq d_{S}\left(\mathcal{F}_{1}, \mathcal{F}_{2}\right)$. For two KBs $\mathcal{K}_{1}, \mathcal{K}_{2}$ and $\mathcal{S}=\operatorname{sig}\left(\mathcal{K}_{1} \cup \mathcal{K}_{2}\right), \mathrm{MD}_{F}\left(\mathcal{K}_{1}, \mathcal{K}_{2}\right)$ is the set of all minimal S-distances (by comparing both components) between the featured models of $\mathcal{K}_{1}$ and $\mathcal{K}_{2}$ :

$$
\operatorname{MD}_{F}\left(\mathcal{K}_{1}, \mathcal{K}_{2}\right)=\min _{\subseteq}\left(\left\{d_{S}\left(\mathcal{F}_{1}, \mathcal{F}_{2}\right) \mid \mathcal{F}_{1} \in \operatorname{FM}\left(\mathcal{K}_{1}\right) \text { and } \mathcal{F}_{2} \in \operatorname{FM}\left(\mathcal{K}_{2}\right)\right\}\right) .
$$

While the above measurement consider both components of S-distances, there are other ways of measurement, that is, by comparing only the first or the second component of S-distances. In particular, define

$$
\begin{aligned}
& \operatorname{MD}_{T}\left(\mathcal{K}_{1}, \mathcal{K}_{2}\right)=\min _{\subseteq}\left(\left\{\Xi_{1} \triangle \Xi_{2} \mid\left\langle\Xi_{1}, \mathcal{H}_{1}\right\rangle \in \operatorname{FM}\left(\mathcal{K}_{1}\right) \text { and }\left\langle\Xi_{2}, \mathcal{H}_{2}\right\rangle \in \operatorname{FM}\left(\mathcal{K}_{2}\right)\right\}\right), \\
& \operatorname{MD}_{H}\left(\mathcal{K}_{1}, \mathcal{K}_{2}\right)=\min _{\subseteq}\left(\left\{\mathcal{H}_{1} \triangle \mathcal{H}_{2} \mid\left\langle\Xi_{1}, \mathcal{H}_{1}\right\rangle \in \operatorname{FM}\left(\mathcal{K}_{1}\right) \text { and }\left\langle\Xi_{2}, \mathcal{H}_{2}\right\rangle \in \operatorname{FM}\left(\mathcal{K}_{2}\right)\right\}\right) .
\end{aligned}
$$

For a pair of featured models $\left\langle\Xi_{1}, \mathcal{H}_{1}\right\rangle$ and $\left\langle\Xi_{2}, \mathcal{H}_{2}\right\rangle$ of $\mathcal{K}_{1}$ and $\mathcal{K}_{2}$, respectively, we say the $\mathrm{S}$-distance between them is $F$-minimal (T-minimal, or $H$-minimal) between $\mathcal{K}_{1}$ and $\mathcal{K}_{2}$ if $\left\langle\Xi_{1} \triangle \Xi_{2}, \mathcal{H}_{1} \triangle \mathcal{H}_{2}\right\rangle \in \operatorname{MD}_{F}\left(\mathcal{K}_{1}, \mathcal{K}_{2}\right)$ (resp., $\Xi_{1} \triangle \Xi_{2} \in \operatorname{MD}_{T}\left(\mathcal{K}_{1}, \mathcal{K}_{2}\right), \mathcal{H}_{1} \triangle \mathcal{H}_{2} \in$ $\left.\operatorname{MD}_{H}\left(\mathcal{K}_{1}, \mathcal{K}_{2}\right)\right)$. Again, we may not mention $\mathcal{K}_{1}$ and $\mathcal{K}_{2}$ if they are clear from the context.

To define our second revision operator using S-distances, we say a featured model $\mathcal{F}^{\prime}$ of $\mathcal{K}^{\prime}$ is $S$-closest to $\mathcal{K}$ if there exists a featured model $\mathcal{F}$ of $\mathcal{K}$ such that the S-distance between $\mathcal{F}$ and $\mathcal{F}^{\prime}$ is both F-minimal and $\mathrm{H}$-minimal between $\mathcal{K}$ and $\mathcal{K}^{\prime}$. In this case, we simply say that the S-distance between $\mathcal{F}$ and $\mathcal{F}^{\prime}$ is minimal. After presenting the definition of our second revision operator, we will show why some (simpler) alternatives cannot work.

Definition 4.10 (S-Revision). Let $\mathcal{K}, \mathcal{K}^{\prime}$ be two $\mathrm{KBs}$ and $\mathcal{S}=\operatorname{sig}\left(\mathcal{K} \cup \mathcal{K}^{\prime}\right)$. The symmetric difference-based revision (S-revision) of $\mathcal{K}$ by $\mathcal{K}^{\prime}$ is a DKB $\mathcal{K} \circ_{S} \mathcal{K}^{\prime}$, such that $\mathrm{FM}\left(\mathcal{K} \circ_{S} \mathcal{K}^{\prime}\right)=$ $\operatorname{FM}\left(\mathcal{K}^{\prime}\right)$ if $\operatorname{FM}(\mathcal{K})=\emptyset$, and otherwise $\operatorname{FM}\left(\mathcal{K} \circ_{S} \mathcal{K}^{\prime}\right)$ is the set of all featured models in $\operatorname{FM}\left(\mathcal{K}^{\prime}\right)$ that are $\mathrm{S}$-closest to $\mathcal{K}$.

Like P-revision, the result of S-revision is well defined and may not be expressible as a single DLLite $_{\text {bool }}^{\mathcal{N}}$ KB in general, which can also be seen from Example 4.5 .

In the following example, we demonstrate S-revision with our running example, which shows that $\circ_{S}$ behaves better than $\circ_{P}$ under the MA in this case. 
Example 4.11 (Cont. Exampe 4.2). Consider the $\mathrm{KBs} \mathcal{K}$ and $\mathcal{K}^{\prime}$, and their respective featured models $\mathcal{F}$ and $\mathcal{F}^{\prime}$ in Example 4.2 The S-distance between $\mathcal{F}$ and $\mathcal{F}^{\prime}$ is not minimal, and $\mathcal{F}^{\prime}$ is not a featured model of $\mathcal{K} \circ_{S} \mathcal{K}^{\prime}$. This can be seen as follows. Take a featured model $\mathcal{F}_{1}=\left\langle\left\{\tau_{1}, \tau_{5}, \tau_{6}\right\}, \mathcal{H}_{1}\right\rangle$ of $\mathcal{K}$ and a featured model $\mathcal{F}_{1}^{\prime}=\left\langle\left\{\tau_{3}, \tau_{5}, \tau_{6}\right\}, \mathcal{H}_{2}\right\rangle$ of $\mathcal{K}^{\prime}$, where $\tau_{5}=\left\{\exists\right.$ hasStaffID ${ }^{-}$, ID $\}, \tau_{6}=\{\exists$ hasStaffID $\}$,

$$
\begin{aligned}
& \mathcal{H}_{1}=\left\{\text { PhDStudent(John), Student(John), ᄏhasStaffID }{ }^{-}(\text {S0564), ID(S0564) }\}\right. \text {, and } \\
& \mathcal{H}_{1}^{\prime}=\left\{\text { PhDStudent(John), Student(John), ヨhasStaffID }{ }^{-}(\text {S0564), ID(S0564), }\right. \\
& \exists \text { hasStaffID(John), hasStaffID(John, S0564)\}. }
\end{aligned}
$$

Then, we have that $\mathcal{H}_{1} \triangle \mathcal{H}_{1}^{\prime}=\{\exists$ hasStaffID(John), hasStaffID(John, S0564) $\}$ is smaller than $\mathcal{H} \triangle \mathcal{H}^{\prime}=\left\{\exists\right.$ hasStaffID $($ John $)$, hasStaffID (John, S0564), ᄏhasStaffID ${ }^{-}($S0564) $\}$, and also $d_{S}\left(\mathcal{F}_{1}, \mathcal{F}_{1}^{\prime}\right)=\left\langle\left\{\tau_{1}, \tau_{3}\right\}, \mathcal{H}_{1} \triangle \mathcal{H}_{1}^{\prime}\right\rangle$ is smaller than $d_{S}\left(\mathcal{F}, \mathcal{F}^{\prime}\right)=\left\langle\left\{\tau_{1}, \tau_{2}, \tau_{3}, \tau_{4}\right\}, \mathcal{H} \triangle \mathcal{H}^{\prime}\right\rangle$. Indeed, the S-distance between $\mathcal{F}_{1}$ and $\mathcal{F}_{1}^{\prime}$ is minimal, and thus $\mathcal{F}_{1}^{\prime}$ is a featured model of $\mathcal{K} \circ_{S} \mathcal{K}^{\prime}$. Also, $d_{S}\left(\mathcal{F}_{1}, \mathcal{F}_{1}^{\prime}\right)$ is the only minimal $\mathrm{S}$-distance.

We can show that the MA of $\mathcal{K} \circ_{S} \mathcal{K}^{\prime}$ is as follows, where Student $\sqsubseteq \neg \exists$ hasStaffID is revised (and weakened) to be Student $\sqcap \exists$ hasStaffID $\sqsubseteq$ PhDStudent.

$$
\begin{aligned}
& \left\langle\left\{\text { PhDStudent } \sqsubseteq \text { Student, Student } \sqcap \text { ID } \sqsubseteq \perp, \exists \text { hasStaffID }{ }^{-} \sqsubseteq\right.\right. \text { ID, } \\
& \text { PhDStudent } \sqsubseteq \exists \text { hasStaffID, Student } \sqcap \exists \text { hasStaffID } \sqsubseteq \text { PhDStudent }\}, \\
& \{\text { PhDStudent(John), hasStaffID(John, S0564) }\}\rangle .
\end{aligned}
$$

In what follows, we present some alternatives for defining a revision operator based on symmetric differences and discuss why they are less desired than S-revision (Definition 4.10). First, we could define the featured models of the revision to be $\operatorname{FM}\left(\mathcal{K} \circ \mathcal{K}^{\prime}\right)=\left\{\mathcal{F}^{\prime} \in \operatorname{FM}\left(\mathcal{K}^{\prime}\right) \mid\right.$ there exists $\mathcal{F} \in$ $\operatorname{FM}(\mathcal{K})$ s.t. $d_{S}\left(\mathcal{F}, \mathcal{F}^{\prime}\right)$ is F-minimal $\}(*)$. However, an observation is that the revision operator defined this way does not function well under MA. This is because the approximation may recruit many undesired featured models that are not close to $\mathcal{K}$ in any aspect. To see this, suppose a featured model $\left\langle\Xi_{m}, \mathcal{H}_{n}\right\rangle$ of $\mathcal{K}^{\prime}$ has an S-distance (to some featured model of $\mathcal{K}$ ) that is F-minimal and not H-minimal; and another featured model $\left\langle\Xi_{n}, \mathcal{H}_{m}\right\rangle$ of $\mathcal{K}^{\prime}$ has an S-distance that is F-minimal and not T-minimal. Both $\left\langle\Xi_{m}, \mathcal{H}_{n}\right\rangle$ and $\left\langle\Xi_{n}, \mathcal{H}_{m}\right\rangle$ are featured models of the revision defined via (*), then from Corollary 3.27, the featured interpretation $\left\langle\Xi_{m} \cup \Xi_{n}, \mathcal{H}_{n}\right\rangle$ is in the $\bigoplus$ closure of any set containing both $\left\langle\Xi_{m}, \mathcal{H}_{n}\right\rangle$ and $\left\langle\Xi_{n}, \mathcal{H}_{m}\right\rangle$, and is also a featured model of the revision under MA. Yet $\left\langle\Xi_{m} \cup \Xi_{n}, \mathcal{H}_{n}\right\rangle$ may not have an F-minimal S-distance.

Example 4.12. Consider two KBs $\mathcal{K}=\langle\{A \sqsubseteq B\},\{A(a)\}\rangle$ and $\mathcal{K}^{\prime}=\langle\{A \sqsubseteq \neg B\}$, $\{\neg B(a)\}\rangle$, two featured models of $\mathcal{K}^{\prime}, \mathcal{F}_{1}^{\prime}=\left\langle\left\{\tau_{1}\right\}, \bar{\emptyset}\right\rangle$ and $\mathcal{F}_{2}^{\prime}=\left\langle\left\{\tau_{2}\right\},\{A(a)\}\right\rangle$, and two featured models of $\mathcal{K}, \mathcal{F}_{1}=\left\langle\left\{\tau_{1}, \tau_{3}\right\},\{A(a), B(a)\}\right\rangle$ and $\mathcal{F}_{2}=\left\langle\left\{\tau_{3}\right\},\{A(a), B(a)\}\right\rangle$, where $\tau_{1}=\emptyset$, $\tau_{2}=\{A\}$, and $\tau_{3}=\{A, B\}$.

Then, $\mathcal{F}_{1}^{\prime}$ has an $\mathrm{S}$-distance to $\mathcal{F}_{1}$ that is F-minimal but not $\mathrm{H}$-minimal (compared to the $\mathrm{S}$ distance between $\mathcal{F}_{2}$ and $\mathcal{F}_{2}^{\prime}$ ); also, $\mathcal{F}_{2}^{\prime}$ has an S-distance to $\mathcal{F}_{2}$ that is $\mathrm{F}$-minimal but not T-minimal (compared to the S-distance between $\mathcal{F}_{1}$ and $\mathcal{F}_{1}^{\prime}$ ). Both $\mathcal{F}_{1}^{\prime}$ and $\mathcal{F}_{2}^{\prime}$ are featured models of the revision defined via (*). By Corollary 3.27 featured interpretation $\mathcal{F}^{\prime \prime}=\left\langle\left\{\tau_{1}, \tau_{2}\right\}, \emptyset\right\rangle$ is in the $\bigoplus$ closure of any set containing both $\mathcal{F}_{1}^{\prime}$ and $\mathcal{F}_{2}^{\prime}$, and is a featured model of the revision under MA, whereas it does not have an F-minimal S-distance to any featured model of $\mathcal{K}$. Indeed, the MA of the revision defined via (*) is exactly $\mathcal{K}^{\prime}$, and the knowledge in $\mathcal{K}$ is totally lost.

In contrast, by Definition 4.10 the MA of S-revision for the KBs is the KB $\langle\{A \sqsubseteq \neg B\},\{A(a)$, $\neg B(a)\}\rangle$, which preserves knowledge $A(a)$ from the initial KB.

We are prompted to restrict the condition in $(*)$, and define $\operatorname{FM}\left(\mathcal{K} \circ \mathcal{K}^{\prime}\right)=\left\{\mathcal{F}^{\prime} \in\right.$ $\operatorname{FM}\left(\mathcal{K}^{\prime}\right) \mid$ there exists $\mathcal{F} \in \mathrm{FM}(\mathcal{K})$ s.t. $d_{S}\left(\mathcal{F}, \mathcal{F}^{\prime}\right)$ is both T-minimal and H-minimal $\}$ (**). Note that an S-distance that is both T-minimal and H-minimal is also F-minimal. However, the above 
condition is too strong and the set $\mathrm{FM}\left(\mathcal{K} \circ \mathcal{K}^{\prime}\right)$ may be empty, and as a result, the defined revision could be inconsistent. Take the KBs in Example 4.12, the revision defined by $(* *)$ is inconsistent.

A third way to define the revision is to weaken the restrictions in (**) and define $\operatorname{FM}\left(\mathcal{K} \circ \mathcal{K}^{\prime}\right)=$ $\left\{\mathcal{F}^{\prime} \in \mathrm{FM}\left(\mathcal{K}^{\prime}\right) \mid\right.$ there exists $\mathcal{F} \in \mathrm{FM}(\mathcal{K})$ s.t. $d_{S}\left(\mathcal{F}, \mathcal{F}^{\prime}\right)$ is both F-minimal and T-minimal $\}$ (***). This definition helps to resolve the inconsistency problem, but often results in incoherent revision. Recall that an incoherent KB is one with unsatisfiable concepts or roles. When the extension of the initial TBox with the new TBox is incoherent, the TBox of the revision defined by $(* * *)$ is exactly the incoherent extension. Again, take the KBs in Example 4.12, the MA of the revision defined by (***) is $\langle\{A \sqsubseteq B, A \sqsubseteq \neg B\},\{\neg B(a)\}\rangle$, which is incoherent, whereas the MA of S-revision is coherent (see Example 4.12).

\subsection{AGM Postulates for Ontology Revision}

In this section, we demonstrate the suitability of our revision operators against the standard AGM postulates [Alchourrón et al. 1985, Katsuno and Mendelzon 1991]. In [Katsuno and Mendelzon [1991], a KB is represented as (the conjunction of) a finite set of propositional sentences rather than a logically closed set, which is more suitable for the DL setting. Hence, we adopt the six postulates (R1)-(R6) in [Katsuno and Mendelzon 1991]. The postulates for propositional belief revision have been adapted to DLs, e.g., [Qi et al. 2006], in which the postulates are formulated in terms of models of KBs. In the following, we reformulate the AGM postulates in term of KBs and entailment relations, in a manner analogous to the formulation in [Katsuno and Mendelzon 1991].

(RI). $\mathcal{K} \circ \mathcal{K}^{\prime}=\mathcal{K}^{\prime}$

$(R 2)$. if $\mathcal{K} \cup \mathcal{K}^{\prime}$ is consistent, then $\mathcal{K} \circ \mathcal{K}^{\prime} \equiv \mathcal{K} \cup \mathcal{K}^{\prime}$;

(R3). if $\mathcal{K}^{\prime}$ is consistent, then $\mathcal{K} \circ \mathcal{K}^{\prime}$ is consistent;

$(R 4)$. if $\mathcal{K}_{1} \equiv \mathcal{K}_{2}$ and $\mathcal{K}_{1}^{\prime} \equiv \mathcal{K}_{2}^{\prime}$, then $\mathcal{K}_{1} \circ \mathcal{K}_{1}^{\prime} \equiv \mathcal{K}_{2} \circ \mathcal{K}_{2}^{\prime}$;

(R5). $\left(\mathcal{K} \circ \mathcal{K}^{\prime}\right) \cup \mathcal{K}^{\prime \prime} \models \mathcal{K} \circ\left(\mathcal{K}^{\prime} \cup \mathcal{K}^{\prime \prime}\right)$;

(R6). if $\left(\mathcal{K} \circ \mathcal{K}^{\prime}\right) \cup \mathcal{K}^{\prime \prime}$ is consistent, then $\mathcal{K} \circ\left(\mathcal{K}^{\prime} \cup \mathcal{K}^{\prime \prime}\right) \models\left(\mathcal{K} \circ \mathcal{K}^{\prime}\right) \cup \mathcal{K}^{\prime \prime}$.

The first postulate (R1) guarantees that the new KB has a higher priority over the old $\mathrm{KB}$ and thus only the old KB is revised when inconsistency occurs in the amalgamation of the two KBs. This is also a major difference of revision from merging. The second postulate (R2) says that no revision is needed if the amalgamation of the two given KBs is consistent. The third postulate (R3) ensures that the result of revision is consistent provided that the new KB is consistent. The fourth postulate (R4) shows that the revision operator is syntax-independent, that is, revising equivalent KBs with equivalent new KBs has equivalent results. The fifth postulate (R5) requires that the revision by a smaller new KB preserves more information than the revision by a larger new $\mathrm{KB}$, and together with (R6), they enforce minimal change by inducing implicitly a total pre-order over the models. While (R1)-(R4) have been well accepted, there is still no consensus about the necessity of the postulates (R5) and (R6) [Creignou et al. 2012]. Moreover, Satoh's revision operator and its variants usually do not obey (R6).

Since the revision operators $\circ_{P}$ and $\circ_{S}$ introduced in the last section are based on Satoh's distance, they satisfy the first five postulates but not the last postulate. However, both of the revision operators satisfy a weaker version of (R6) proposed in [Katsuno and Mendelzon 1991].

$\left(R 6^{\prime}\right)$. if $\left(\mathcal{K} \circ \mathcal{K}^{\prime}\right)=\mathcal{K}^{\prime \prime}$, then $\mathcal{K} \circ\left(\mathcal{K}^{\prime} \cup \mathcal{K}^{\prime \prime}\right) \models\left(\mathcal{K} \circ \mathcal{K}^{\prime}\right) \cup \mathcal{K}^{\prime \prime}$.

THEOREM 4.13. Both P-revision and S-revision satisfy the postulates (R1)-(R5), (R6').

If the results of revision are replaced with their MAs in the postulates, then both the operators satisfy postulates $(R 1)-(R 4)$.

Proof Let $X=P$ or $S$. The following proof works both for P-revision and S-revision.

(R1) By the definition of X-revision, $\operatorname{FM}\left(\mathcal{K} \circ_{X} \mathcal{K}^{\prime}\right) \subseteq \mathrm{FM}\left(\mathcal{K}^{\prime}\right)$. By Theorem $3.21 \mathcal{K} \circ_{X} \mathcal{K}^{\prime} \models \mathcal{K}^{\prime}$. 
From the definition of the $\mathrm{MA}, \operatorname{FM}\left(\mathcal{K} \circ_{X} \mathcal{K}^{\prime}\right) \subseteq \operatorname{FM}\left(\operatorname{MA}\left(\mathcal{K} \circ_{X} \mathcal{K}^{\prime}\right)\right)$. As $\operatorname{FM}\left(\mathcal{K} \circ_{X} \mathcal{K}^{\prime}\right) \subseteq \operatorname{FM}\left(\mathcal{K}^{\prime}\right)$, by Proposition 3.18. $\mathrm{FM}\left(\mathcal{K} \circ_{X} \mathcal{K}^{\prime}\right) \subseteq \operatorname{FM}\left(\operatorname{MA}\left(\mathcal{K} \circ_{X} \mathcal{K}^{\prime}\right) \cup \mathcal{K}^{\prime}\right)$. By the uniqueness of the MA, $\operatorname{FM}\left(\operatorname{MA}\left(\mathcal{K} \circ_{X} \mathcal{K}^{\prime}\right)\right)=\operatorname{FM}\left(\operatorname{MA}\left(\mathcal{K} \circ_{X} \mathcal{K}^{\prime}\right) \cup \mathcal{K}^{\prime}\right)$. That is, $\operatorname{MA}\left(\mathcal{K} \circ_{X} \mathcal{K}^{\prime}\right)=\mathcal{K}^{\prime}$.

(R2) If $\mathcal{K} \cup \mathcal{K}^{\prime}$ is consistent, $\operatorname{FM}\left(\mathcal{K}^{\prime}\right) \cap \mathrm{FM}(\mathcal{K}) \neq \emptyset$. From the definition of $X$-revision, the featured models of $\mathcal{K} \circ_{X} \mathcal{K}^{\prime}$ are exactly those in $\operatorname{FM}\left(\mathcal{K}^{\prime}\right) \cap \operatorname{FM}(\mathcal{K})$. By Theorem 3.21, $\mathcal{K} \circ_{X} \mathcal{K}^{\prime} \equiv \mathcal{K} \cup \mathcal{K}^{\prime}$.

By the uniqueness of the MA, $\operatorname{MA}\left(\mathcal{K} \circ_{X} \mathcal{K}^{\prime}\right) \equiv \mathcal{K} \cup \mathcal{K}^{\prime}$.

(R3) If $\mathcal{K}^{\prime}$ is consistent, then $\operatorname{FM}\left(\mathcal{K}^{\prime}\right) \neq \emptyset$. From the definition of X-revision, $\operatorname{FM}\left(\mathcal{K} \circ_{X} \mathcal{K}^{\prime}\right) \neq \emptyset$. By Theorem 3.20, $\mathcal{K} \circ_{X} \mathcal{K}^{\prime}$ is consistent.

From the definition of the $\mathrm{MA}, \operatorname{FM}\left(\operatorname{MA}\left(\mathcal{K} \circ_{X} \mathcal{K}^{\prime}\right)\right) \neq \emptyset$. Similarly, $\operatorname{MA}\left(\mathcal{K} \circ_{X} \mathcal{K}^{\prime}\right)$ is consistent.

(R4) It is straightforward from the definition of X-revision and Theorem 3.21

(R5) For each $\mathcal{F}^{\prime} \in \operatorname{FM}\left(\left(\mathcal{K} \circ_{X} \mathcal{K}^{\prime}\right) \cup \mathcal{K}^{\prime \prime}\right)$, by Proposition 3.18, $\mathcal{F}^{\prime} \in \operatorname{FM}\left(\mathcal{K} \circ_{X} \mathcal{K}^{\prime}\right)$ and $\mathcal{F}^{\prime} \in \operatorname{FM}\left(\mathcal{K}^{\prime \prime}\right)$. From the definition of X-revision, $\mathcal{F}^{\prime} \in \operatorname{FM}\left(\mathcal{K}^{\prime}\right)$, and there exists $\mathcal{F} \in \operatorname{FM}(\mathcal{K})$ such that the $\mathrm{X}$-distance between $\mathcal{F}$ and $\mathcal{F}^{\prime}$ is minimal between $\mathcal{K}$ and $\mathcal{K}^{\prime}$. That is, there do not exist $\mathcal{F}_{1} \in \mathrm{FM}(\mathcal{K})$ and $\mathcal{F}_{1}^{\prime} \in \mathrm{FM}\left(\mathcal{K}^{\prime}\right)$ that witness the non-minimality of the X-distance between $\mathcal{F}$ and $\mathcal{F}^{\prime}$. Clearly, there do not exist such $\mathcal{F}_{1}$ and $\mathcal{F}_{1}^{\prime}$ in $\operatorname{FM}(\mathcal{K})$ and $\operatorname{FM}\left(\mathcal{K}^{\prime}\right) \cap \operatorname{FM}\left(\mathcal{K}^{\prime \prime}\right)$, respectively. That is, the $\mathrm{X}$-distance between $\mathcal{F}$ and $\mathcal{F}^{\prime}$ is minimal between $\mathcal{K}$ and $\mathcal{K}^{\prime} \cup \mathcal{K}^{\prime \prime}$. Since $\mathcal{F}^{\prime} \in \operatorname{FM}\left(\mathcal{K}^{\prime}\right)$ and $\mathcal{F}^{\prime} \in \operatorname{FM}\left(\mathcal{K}^{\prime \prime}\right)$, by Proposition 3.18, $\mathcal{F}^{\prime} \in \operatorname{FM}\left(\mathcal{K}^{\prime} \cup \mathcal{K}^{\prime \prime}\right)$. Thus, $\mathcal{F}^{\prime} \in \operatorname{FM}\left(\mathcal{K} \circ_{X}\left(\mathcal{K}^{\prime} \cup \mathcal{K}^{\prime \prime}\right)\right)$. That is, $\operatorname{FM}\left(\left(\mathcal{K} \circ_{X} \mathcal{K}^{\prime}\right) \cup \mathcal{K}^{\prime \prime}\right) \subseteq \operatorname{FM}\left(\mathcal{K} \circ_{X}\left(\mathcal{K}^{\prime} \cup \mathcal{K}^{\prime \prime}\right)\right)$, and by Theorem 3.21 . $\left(\mathcal{K} \circ_{X} \mathcal{K}^{\prime}\right) \cup \mathcal{K}^{\prime \prime} \mid=$ $\mathcal{K} \circ_{X}\left(\mathcal{K}^{\prime} \cup \mathcal{K}^{\prime \prime}\right)$.

(R6') For each $\mathcal{F}^{\prime} \in \mathrm{FM}\left(\mathcal{K} \circ_{X}\left(\mathcal{K}^{\prime} \cup \mathcal{K}^{\prime \prime}\right)\right)$, from the definition of X-revision, $\mathcal{F}^{\prime} \in \mathrm{FM}\left(\mathcal{K}^{\prime} \cup \mathcal{K}^{\prime \prime}\right)$. By Proposition 3.18, $\mathcal{F}^{\prime} \in \operatorname{FM}\left(\mathcal{K}^{\prime}\right)$ and $\mathcal{F}^{\prime} \in \mathrm{FM}\left(\mathcal{K}^{\prime \prime}\right)$. By the definition of X-revision, there exists $\mathcal{F} \in \mathrm{FM}(\mathcal{K})$ such that the $\mathrm{X}$-distance between $\mathcal{F}$ and $\mathcal{F}^{\prime}$ is minimal between $\mathcal{K}$ and $\mathcal{K}^{\prime} \cup \mathcal{K}^{\prime \prime}$. That is, there exist no $\mathcal{F}_{1} \in \mathrm{FM}(\mathcal{K})$ and $\mathcal{F}_{1}^{\prime} \in \operatorname{FM}\left(\mathcal{K}^{\prime}\right) \cap \operatorname{FM}\left(\mathcal{K}^{\prime \prime}\right)$ that witness the non-minimality of the X-distance between $\mathcal{F}$ and $\mathcal{F}^{\prime}$. We want to show that there exist no such $\mathcal{F}_{1}$ and $\mathcal{F}_{1}^{\prime}$ in $\operatorname{FM}(\mathcal{K})$ and $\operatorname{FM}\left(\mathcal{K}^{\prime}\right)$, respectively. Towards a contradiction, suppose such $\mathcal{F}_{1}$ and $\mathcal{F}_{1}^{\prime}$ exist; and without loss of generality, we can assume the X-distance between $\mathcal{F}_{1}$ and $\mathcal{F}_{1}^{\prime}$ is minimal between $\mathcal{K}$ and $\mathcal{K}^{\prime}$ as otherwise they could be replaced with another pair of featured interpretations with a minimal Xdistance. In this case, $\mathcal{F}_{1}^{\prime} \in \operatorname{FM}\left(\mathcal{K} \circ_{X} \mathcal{K}^{\prime}\right)$. Since $\mathcal{K} \circ_{X} \mathcal{K}^{\prime}=\mathcal{K}^{\prime \prime}, \mathcal{F}_{1}^{\prime} \in \operatorname{FM}\left(\mathcal{K}^{\prime \prime}\right)$. Note that we assume $\mathcal{F}_{1}^{\prime} \in \operatorname{FM}\left(\mathcal{K}^{\prime}\right)$, and by Proposition 3.18, $\mathcal{F}_{1}^{\prime} \in \operatorname{FM}\left(\mathcal{K}^{\prime} \cup \mathcal{K}^{\prime \prime}\right)$. This contradicts the fact that the X-distance between $\mathcal{F}$ and $\mathcal{F}^{\prime}$ is minimal between $\mathcal{K}$ and $\mathcal{K}^{\prime} \cup \mathcal{K}^{\prime \prime}$. Thus, we have shown that the X-distance between $\mathcal{F}$ and $\mathcal{F}^{\prime}$ is minimal between $\mathcal{K}$ and $\mathcal{K}^{\prime}$. By the definition of X-revision, $\mathcal{F} \in \mathrm{FM}\left(\mathcal{K} \circ_{X} \mathcal{K}^{\prime}\right)$. Since $\mathcal{F} \in \mathrm{FM}\left(\mathcal{K}^{\prime \prime}\right)$, by Proposition 3.18, $\mathcal{F} \in \operatorname{FM}\left(\left(\mathcal{K} \circ_{X} \mathcal{K}^{\prime}\right) \cup \mathcal{K}^{\prime \prime}\right)$. That is, $\operatorname{FM}\left(\mathcal{K} \circ_{X}\left(\mathcal{K}^{\prime} \cup \mathcal{K}^{\prime \prime}\right)\right) \subseteq \operatorname{FM}\left(\left(\mathcal{K} \circ_{X} \mathcal{K}^{\prime}\right) \cup \mathcal{K}^{\prime \prime}\right)$, and by Theorem 3.21, $\mathcal{K} \circ_{X}\left(\mathcal{K}^{\prime} \cup \mathcal{K}^{\prime \prime}\right)=$ $\left(\mathcal{K} \circ_{X} \mathcal{K}^{\prime}\right) \cup \mathcal{K}^{\prime \prime}$

The following example shows that (R5) and (R6) are not satisfied by S-revision under the MA. The example can be modified to show that (R6) is not satisfied by either of our two revision operators, and P-revision satisfies neither (R5) nor (R6) under the MA.

Example 4.14. Let $\mathcal{K}=\langle\{A \sqsubseteq B, B \sqsubseteq C\},\{A(a)\}\rangle, \mathcal{K}^{\prime}=\langle\{B \sqsubseteq A, A \sqsubseteq \neg C\},\{(A \sqcup$ $C)(a)\}\rangle$, and $\mathcal{K}^{\prime \prime}=\langle\emptyset,\{\neg B \overline{(a)}\}\rangle$. Then $\operatorname{MA}\left(\mathcal{K} \circ_{S} \mathcal{K}^{\prime}\right)=\langle\{A \sqsubseteq B, \bar{B} \sqsubseteq A, A \sqsubseteq$ $\neg C\},\{(A \sqcup C)(a)\}\rangle$, and after combined with $\mathcal{K}^{\prime \prime},(A \sqcup C)(a)$ in the ABox is replaced with $C(a)$. However, $\operatorname{MA}\left(\mathcal{K} \circ_{S}\left(\mathcal{K}^{\prime} \cup \mathcal{K}^{\prime \prime}\right)\right)=\langle\{B \sqsubseteq C, B \sqsubseteq A, A \sqsubseteq \neg C\},\{(A \sqcup C)(a)\}\rangle$. That is, $\left(\mathrm{MA}\left(\mathcal{K} \circ_{S} \mathcal{K}^{\prime}\right)\right) \cup \mathcal{K}^{\prime \prime} \not \operatorname{MA}\left(\mathcal{K} \circ_{S}\left(\mathcal{K}^{\prime} \cup \mathcal{K}^{\prime \prime}\right)\right)$ and $\operatorname{MA}\left(\mathcal{K} \circ_{S}\left(\mathcal{K}^{\prime} \cup \mathcal{K}^{\prime \prime}\right)\right) \not \forall\left(\operatorname{MA}\left(\mathcal{K} \circ_{S} \mathcal{K}^{\prime}\right)\right) \cup \mathcal{K}^{\prime \prime}$.

\subsection{Application Case Study}

We illustrate the practical applications of our approach to a Medical Ontology Enrichment scenario. NCI Thesaurus (NCI) [Hartel et al. 2005] is a well known ontology for medical applications. An ontology $\mathcal{K}$ based on NCI consists of a TBox $\mathcal{T}$ containing axioms describing medical terms and an ABox $\mathcal{A}$ containing medical data. From time to time, new medical terms, denoted $\mathcal{T}^{\prime}$, are reported in the literature and new data, denoted $\mathcal{A}^{\prime}$, are identified from practical experience. We then need to enrich the ontology $\mathcal{K}$ by revising it using the ontology $\mathcal{K}^{\prime}=\left\langle\mathcal{T}^{\prime}, \mathcal{A}^{\prime}\right\rangle$. 
We now show an example of revising an NCI ontology using our revision operators. We focus on a fragment $\mathcal{K}$ of NCI concerning respiratory and thoracic disorders and their associated anatomic locations. This fragment consists of the concepts Respiratory_and_Thoracic_Disorder (RT_Disorder), Respiratory_System, Cardiovascular_System, and Organ_System, as well as a single role that associates diseases to their locations Disease_Has_Associated_Anatomic_Site (has_Site).

\begin{tabular}{|c|c|}
\hline \multicolumn{2}{|r|}{ Initial axioms in the ontology } \\
\hline A1 & RT_Disorder $\sqsubseteq \exists$ has_Site \\
\hline A2 & $\exists$ has_Site ${ }^{-} \sqsubseteq$ Respiratory_System \\
\hline A3 & Respiratory_System $\sqsubseteq$ Organ_System \\
\hline A4 & Cardiovascular_System $\sqsubseteq$ Organ_System \\
\hline A5 & RT_Disorder $\sqcap$ Organ_System $\sqsubseteq \perp$ \\
\hline A6 & Respiratory_System $\sqcap$ Cardiovascular_System $\sqsubseteq \perp$ \\
\hline \multicolumn{2}{|r|}{ Axioms to be incorporated } \\
\hline A7 & Heart_Disease $\sqsubseteq$ RT_Disorder \\
\hline A8 & Heart_Disease $\sqsubseteq \exists$ has_Site \\
\hline A9 & $\exists$ has_Site ${ }^{-} \sqsubseteq$ Cardiovascular_System \\
\hline A10 & Heart_Disease(hd1) \\
\hline A11 & has_Site(hd1, s1) \\
\hline \multicolumn{2}{|r|}{ Axioms generated by revisions } \\
\hline A12 & RT_Disorder $\sqsubseteq \exists U$ \\
\hline A13 & $\exists U^{-} \sqsubseteq$ Respiratory_System \\
\hline A14 & Respiratory_System $\sqcap$ Cardiovascular_System $\sqsubseteq \exists$ has_Site $^{-}$ \\
\hline
\end{tabular}

Fig. 3. Axioms from NCI ontology and those generated by revision.

Some axioms in NCI may be in a more expressive DL and do not have equivalent translations in DL-Lite ${ }_{\text {bool }}^{\mathcal{N}}$. Suppose, the following axiom in NCI, RT_Disorder $\sqsubseteq \exists$ has_Site.Respiratory_System, which states that respiratory and thoracic disorders have associated sites in the respiratory system, is converted (in a rather naive way) into DL-Lite $\mathcal{L}_{\text {bool }}^{\mathcal{N}}$ axioms A1 and A2 in Figure 3 A3-A5 state that the respiratory and cardiovascular systems are both organ systems, and that the class of disorders is disjoint with the class of organ systems. For the purpose of demonstrating the usefulness of our revision operator, A6 is added saying that the respiratory and cardiovascular systems are disjoint, which mimics common mistakes likely to be seen in practice of ontology conceptualization that may lead to contradictions.

Suppose new knowledge about heart diseases, together with some data on instances of heart diseases, is required to be incorporated into the ontology. The new knowledge is expressed as a TBox $\mathcal{T}^{\prime}$ consisting of $\mathrm{A} 7-\mathrm{A} 9$, stating that heart diseases belong to respiratory and thoracic disorders, and heart diseases have associated sites in the cardiovascular system. Again, A8 and A9 are a naive conversion to DL-Lite $\mathcal{B}_{\text {bool }}^{\mathcal{N}}$ (like A1 and A2), yet they are useful to demonstrate the revision operation. Properties of a particular heart disease hd1 are expressed as an ABox $\mathcal{A}^{\prime}$ that consists of A10 and A11, stating that hd 1 is an instance of heart diseases and it is located at site s1.

Adding $\mathcal{K}^{\prime}$ directly into the ontology will cause a contradiction, since in this case both assertions Cardiovascular_System(s1) and Respiratory_System(s1) can be derived, whereas concepts Cardiovascular_System and Respiratory_System have been asserted to be disjoint.

The result of P-revision is a DKB $\left\{\mathcal{K}_{1}, \mathcal{K}_{2}\right\}$, where $\mathcal{K}_{1}$ consists of axioms A1-A 3 , A5, and A7$\mathrm{A} 11$; and $\mathcal{K}_{2}$ consists of axioms $\mathrm{A} 3-\mathrm{A} 11$, and two new axioms A12 and A13 with an auxiliary role $U . \mathcal{K}_{1}$ and $\mathcal{K}_{2}$ can be obtained from $\mathcal{K}$ by forgetting concept Cardiovascular_System and role has_Site, respectively, and adding $\mathcal{K}^{\prime}$. The MA of the result of P-revision consists of axioms A3, A5, and A7-A11. 
On the other hand, while the result of S-revision is difficult to compute (and thus is not shown), the MA of S-revision preserves more initial knowledge than that of P-revision. The MA of S-revision consists of axioms A1, A3-A5, A7-A11, and one revised axiom A14, which is obtained by weakening axiom A6. It says that the respiratory system and the cardiovascular system have a common part (namely s1), with which some respiratory and thoracic disorder is associated.

A comparison between revision results is shown in Figure 4.

\begin{tabular}{|c|llllllllllll|}
\hline KBs & \multicolumn{10}{|c|}{ Axioms } \\
\hline $\mathcal{K}$ & A1 & A2 & A3 & A4 & A5 & A6 & & & & & \\
\hline $\mathcal{K}^{\prime}$ & & & & & & & & A7 & A8 & A9 & A10 & A11 \\
\hline \multirow{2}{*}{$\circ_{S} \mathcal{K}^{\prime}:$} & $\mathcal{K}_{1}$ & A1 & A2 & A3 & & A5 & & A7 & A8 & A9 & A10 & A11 \\
& $\mathcal{K}_{2}$ & A12 & A13 & A3 & A4 & A5 & A6 & A7 & A8 & A9 & A10 & A11 \\
\hline $\operatorname{MA}\left(\mathcal{K} \circ_{P} \mathcal{K}^{\prime}\right)$ & & & & A3 & & A5 & & A7 & A8 & A9 & A10 & A11 \\
\hline $\operatorname{MA}\left(\mathcal{K} \circ_{S} \mathcal{K}^{\prime}\right)$ & A1 & & A3 & A4 & A5 & A14 & A7 & A8 & A9 & A10 & A11 \\
\hline
\end{tabular}

Fig. 4. Comparison of revision results on NCI ontology.

\section{COMPUTATIONAL ISSUES}

In this section, we will study the computational complexity of our revision operators and look into the computation of revision.

\subsection{Computational Complexity}

Let us look at the computational complexity of the revision operators. The complexity class $\Pi_{2}^{P}=$ coNP $^{\mathrm{NP}}$ consists of the decision problems whose complementary problems are solvable by a nondeterministic Turing machine in polynomial time with NP oracles. It is proved in [Eiter and Gottlob 1992 ] that Satoh's revision for propositional logic is $\Pi_{2}^{P}$-complete. The main result in this section shows that our revision in DL-Lite does not raise the complexity.

We consider the following reasoning problem:

Revision Entailment Given two consistent $\mathrm{KBs} \mathcal{K}$ and $\mathcal{K}^{\prime}$ and a concept inclusion or membership assertion $\alpha$, decide whether $\mathcal{K} \circ_{X} \mathcal{K}^{\prime} \models \alpha$ where $X=P$ or $S$.

THEOREM 5.1. The problem of Revision Entailment is $\Pi_{2}^{P}$-complete.

To prove this, we first have a look at the $\Pi_{2}^{P}$ membership of Revision Entailment in propositional logic. For propositional Revision Entailment problem $K * K^{\prime} \models \phi$, the proof of $\Pi_{2}^{P}$ membership consists of two steps: (1) Guess two models $M$ and $M^{\prime}$ of $K$ and $K^{\prime}$, respectively, and check if $M^{\prime} \forall \neq \phi$; and (2) use a NP oracle to verify that there exists no models $M_{1}$ and $M_{1}^{\prime}$ of $K$ and $K^{\prime}$, respectively, such that $d\left(M_{1}, M_{1}^{\prime}\right) \subset d\left(M, M^{\prime}\right)$, where $d\left(M_{1}, M_{2}\right)$ is the distance between the two models $M_{1}$ and $M_{2}$. Both of these two steps can be done in polynomial time, and thus, the overall complexity is in $\Pi_{2}^{P}$.

However, we cannot adopt such a proof for DL-Lite revision in a straightforward manner. It is because, unlike propositional interpretations, the size of a featured interpretation is exponential with respect to the size of the KBs in general. The exponential blow up is mainly caused by the type set $\Xi$, which is a subset of the power set of the basic concepts $B C_{\mathcal{S}}$. In this sense, Theorem 5.1 is not just a simple generalization of the $\Pi_{2}^{P}$-completeness of propositional revision.

A key idea in our proof of Theorem 5.1 is that we only need to guess and verify featured interpretations whose sizes are polynomial with respect to the sizes of the KBs. In particular, we first show that each featured interpretation "contains" a featured interpretation of polynomial size.

LEMMA 5.2. Let $\left|\mathcal{S}_{R}\right|=m$ and $\left|\mathcal{S}_{I}\right|=n$. Given a featured interpretation $\langle\Xi, \mathcal{H}\rangle$, a featured interpretation $\left\langle\Xi^{*}, \mathcal{H}\right\rangle$ exists such that (1) $\Xi^{*} \subseteq \Xi$, (2) $\left|\Xi^{*}\right| \leq 2 m+n$, and (3) $\left\langle\Xi^{*} \cup \Xi^{\prime}, \mathcal{H}\right\rangle$ is a featured interpretation for any type set $\Xi^{\prime} \subseteq \Xi$. 
Intuitively, the set $\Xi^{*}$ in Lemma 5.2 contains all the types in $\Xi$ that are necessary for satisfying the conditions of a featured interpretation (Definition 3.10).

Moreover, we show that when we consider minimal distances, we only need to consider those distances between featured interpretations of polynomial sizes. The following results concern both P-revision and S-revision.

Lemma 5.3. Let $\left|\mathcal{S}_{R}\right|=m,\left|\mathcal{S}_{I}\right|=n$, and $\mathcal{F}_{i}=\left\langle\Xi_{i}, \mathcal{H}_{i}\right\rangle(i=1,2)$ be a pair of featured interpretations. Suppose $\Sigma \in d_{P}\left(\mathcal{F}_{1}, \mathcal{F}_{2}\right)$, then a pair of featured interpretations $\mathcal{F}_{i}^{P}=\left\langle\Xi_{i}^{P}, \mathcal{H}_{i}\right\rangle$ $(i=1,2)$ exist, such that for $i=1,2,(1) \Xi_{i}^{P} \subseteq \Xi_{i},(2)\left|\Xi_{i}^{P}\right| \leq 4 m+2 n$, and (3) $\Sigma \in d_{P}\left(\mathcal{F}_{1}^{P}, \mathcal{F}_{2}^{P}\right)$.

There also exist a pair of featured interpretations $\mathcal{F}_{i}^{S}=\left\langle\Xi_{i}^{S}, \mathcal{H}_{i}\right\rangle(i=1,2)$, such that for $i=1,2$, (1') $\Xi_{i}^{S} \subseteq \Xi_{i}$, (2') $\left|\Xi_{i}^{S}\right| \leq 4 m+2 n$, and (3') $\Xi_{1}^{S} \triangle \Xi_{2}^{S} \subseteq \Xi_{1} \triangle \Xi_{2}$.

The next result states that from a given featured model that is closest to a $\mathrm{KB}$, a featured model of polynomial size can be constructed that is also closest to the KB.

Lemma 5.4. Let $\mathcal{K}$ and $\mathcal{K}^{\prime}$ be two KBs and $\alpha$ an axiom. For $X=P$ or $S$, if a featured model $\mathcal{F} \in \mathrm{FM}\left(\mathcal{K}^{\prime}\right)$ is $X$-closest to $\mathcal{K}$ and does not satisfy $\alpha$, then there exists a featured model $\mathcal{F}^{\prime} \in \mathrm{FM}\left(\mathcal{K}^{\prime}\right)$ of polynomial size that is $X$-closest to $\mathcal{K}$ and does not satisfy $\alpha$.

Given the above lemmas, we are ready to prove Theorem 5.1

Proof of Theorem 5.1 (Hardness) It follows from the complexity of propositional revision. In particular, a propositional formula $\varphi$ can be transformed into a DL-Lite ${ }_{\text {bool }}^{\mathcal{N}}$ concept description $C_{\varphi}$ in a straightforward way: each propositional variable $p$ is replaced by a unique concept name $A_{p}, \wedge$ is replaced with $\sqcap$, and $\vee$ is replaced with $\sqcup$. The revision of $\varphi$ by $\phi$ can be reduced to the revision of DL-Lite bool $^{\mathcal{N}} \operatorname{KB} \mathcal{K}_{\varphi}=\left\langle\emptyset,\left\{C_{\varphi}(a)\right\}\right\rangle$ by $\mathcal{K}_{\phi}=\left\langle\emptyset,\left\{C_{\phi}(a)\right\}\right\rangle$, where $a$ is new individual name. Note that in this case, P-revision and S-revision coincide. That is, $\omega$ is a minimal distance between $\varphi$ and $\phi$ iff $\left\{\left\{A_{p} \mid p \in \omega\right\}\right\}$ is a minimal P-distance between $\mathcal{K}_{\varphi}$ and $\mathcal{K}_{\phi}$, iff $\left\langle\emptyset,\left\{A_{p}(a) \mid p \in \omega\right\}\right\rangle$ is a minimal S-distance. Thus, a Revision Entailment in propositional logic can be reduced to a Revision Entailment problem in DL-Lite ${ }_{\text {bool }}^{\mathcal{N}}$.

(Membership) A decision algorithm for the complementary problem $\mathcal{K} \circ \mathcal{K}^{\prime} \not \models \alpha$ can be described as follows:

Step 1: Guess two featured interpretations $\mathcal{F}$ and $\mathcal{F}^{\prime}$. By Lemma 5.4 it is without loss of generality to assume that the sizes of $\mathcal{F}$ and $\mathcal{F}^{\prime}$ are bounded by a polynomial of $m+n$ where $m=\left|\mathcal{S}_{R}\right|$ and $n=\left|\mathcal{S}_{I}\right|$. Then, (1) check that $\mathcal{F}$ and $\mathcal{F}^{\prime}$ are featured models of $\mathcal{K}$ and $\mathcal{K}^{\prime}$, respectively; and (2) check that $\mathcal{F}^{\prime}$ does not satisfy $\alpha$.

Step 2: Check whether $\mathcal{K}$ is inconsistent, which can be done with an NP-oracle [Artale et al. 2009]. If it is the case, then $\mathcal{F}^{\prime}$ is in $\operatorname{FM}\left(\mathcal{K} \circ \mathcal{K}^{\prime}\right)$ and $\mathcal{K} \circ \mathcal{K}^{\prime} \not \neq \alpha$. Otherwise, check that there does not exist a pair of featured interpretations $\mathcal{F}_{1}$ and $\mathcal{F}_{1}^{\prime}$ such that (i) the sizes of $\mathcal{F}_{1}$ and $\mathcal{F}_{1}^{\prime}$ are bounded by a polynomial of $m+n$; (ii) $\mathcal{F}_{1}$ and $\mathcal{F}_{1}^{\prime}$ are featured models of $\mathcal{K}$ and $\mathcal{K}^{\prime}$, respectively; and (iii) $\mathcal{F}_{1}$ and $\mathcal{F}_{1}^{\prime}$ witness the non-minimality of the P-distance (or S-distance) between $\mathcal{F}$ and $\mathcal{F}^{\prime}$.

Overall, as the sizes of $\mathcal{F}$ and $\mathcal{F}^{\prime}$ are polynomially bounded, the checks in Step 1 (1) and (2) can be done in polynomial time. Step 2 is conducted by an NP-oracle. In particular, the algorithm guesses $\mathcal{F}_{1}$ and $\mathcal{F}_{1}^{\prime}$, and perform checks (i)-(iii) in polynomial time. Thus, the overall complexity of the decision algorithm is in $\Sigma_{2}^{P}$.

\subsection{Revision Algorithms}

In what follows, we introduce a deterministic algorithm for computing the MA of our revisions. This algorithm works for both P-revision and S-revision. From the above discussions on complexity, we observe that it suffices to consider featured models of polynomial sizes (Lemma 5.3, which we will refer to as representative featured models. Let $\left|\mathcal{S}_{R}\right|=m$ and $\left|\mathcal{S}_{I}\right|=n$, then each representative featured model contains at most $4 m+2 n$ types. We have shown that representative featured models cover all minimal distances, i.e., for each minimal distance between two KBs, there is a pair of representative featured models of respective KBs with the same distance. We apply this result in 


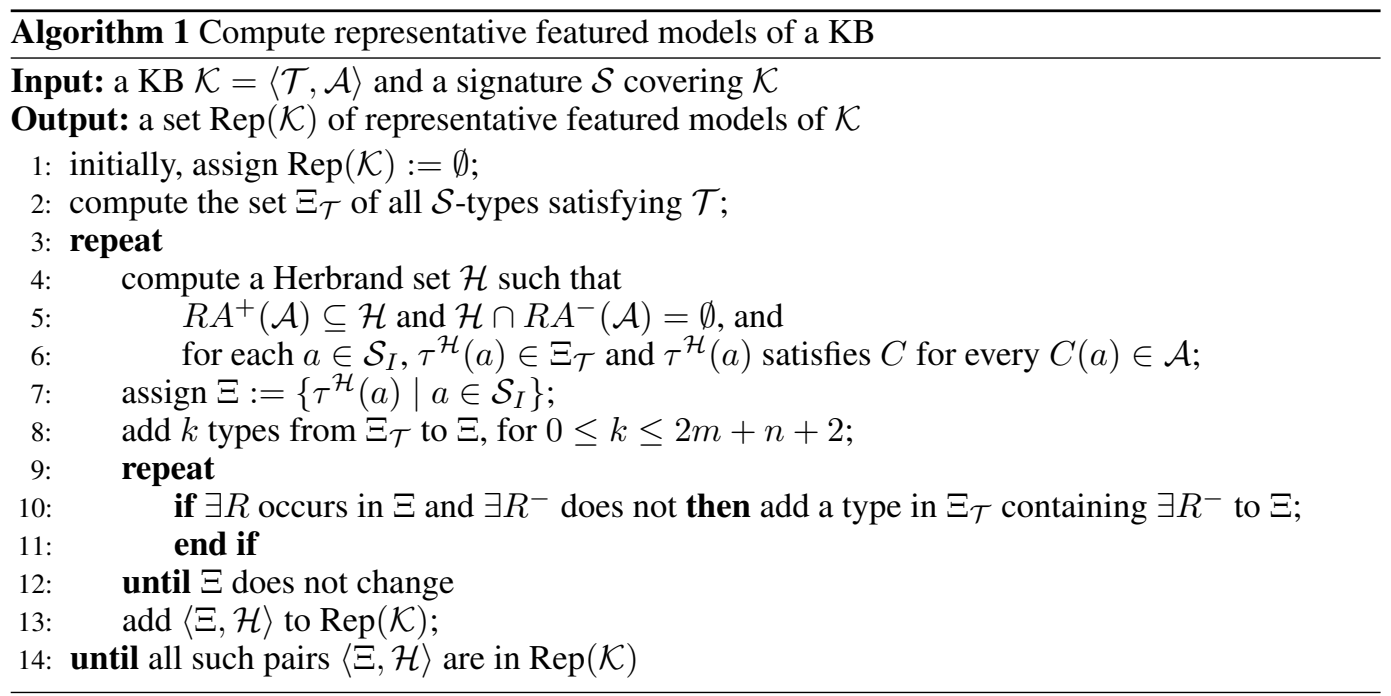

computing the MA of our revisions. In particular, we show that constructing only representative featured models (with minimal distances) is sufficient for computing the MA of revision.

We first introduce a method that computes representative featured models of a KB (ref. Algorithm [1], and then show how the MA of revision can be constructed via such featured models. For an ABox $\mathcal{A}$, denote $R A^{+}(\mathcal{A})=\left\{P(a, b) \mid P(a, b) \in \mathcal{A}, P \in \mathcal{S}_{R}\right.$, and $\left.a, b \in \mathcal{S}_{I}\right\}$ and $R A^{-}(\mathcal{A})=\left\{P(a, b) \mid \neg P(a, b) \in \mathcal{A}, P \in \mathcal{S}_{R}\right.$, and $\left.a, b \in \mathcal{S}_{I}\right\}$.

Algorithm 1 repeatedly adds pairs $\langle\Xi, \mathcal{H}\rangle$ to $\operatorname{Rep}(\mathcal{K})$, and each $\langle\Xi, \mathcal{H}\rangle$ is a featured model of $\mathcal{K}$ with linearly many types. This can be seen as follows: $\langle\Xi, \mathcal{H}\rangle$ is a featured interpretation (Lines 9-12 and Line 7 for Conditions (1) and (2) of Definition 3.10, respectively), and satisfies both $\mathcal{A}$ (Lines 5 and 6 for the satisfaction of the role assertions and the concept assertions in $\mathcal{A}$, respectively) and $\mathcal{T}$ (Lines 6-10, all the types in $\Xi$ are from $\Xi_{\mathcal{T}}$ and hence satisfy $\mathcal{T}$ ). Algorithm 11 returns $\emptyset$ if and only if $\mathcal{K}$ is inconsistent. The number of types in $\Xi$ is at most $4 m+2 n+2$, as the numbers of types added to $\Xi$ are at most $n$ at Line 7, at most $2 m+n+2$ at Line 8 , and at most $2 m$ at Lines 9-12. Note that at Line 8 , every possible set of $k$ types is added to $\Xi$, so that all the desired featured models (containing at most $4 m+2 n+2$ types) are included in $\operatorname{Rep}(\mathcal{K})$. Informally, a maximal $4 m+2 n$ types are needed to cover all minimal distances (see $\mathcal{F}^{P}$ and $\mathcal{F}^{S}$ in Lemma 5.3), and an additional 2 types are needed to ensure Condition (2) in Proposition 5.5 (see its proof).

As the number of representative featured models is (single) exponential w.r.t. the size of the KB, Algorithm 1 takes exponential time to compute $\operatorname{Rep}(\mathcal{K})$. In practice, when Algorithm 1 is used to compute revision, the algorithm can be largely optimised with the information from anther KB $\mathcal{K}^{\prime}$. That is, each time one can generate in parallel a pair of featured models in respectively $\operatorname{Rep}(\mathcal{K})$ and $\operatorname{Rep}\left(\mathcal{K}^{\prime}\right)$, such that the two featured models have a minimal distance. In this way, not all the featured interpretations in $\operatorname{Rep}(\mathcal{K})$ have to be generated.

The following proposition states the desired properties of $\operatorname{Rep}(\mathcal{K})$. For another $\operatorname{KB} \mathcal{K}^{\prime}$ and $X=P$ or $S$, we define the minimal X-distances between $\operatorname{Rep}(\mathcal{K})$ and $\operatorname{Rep}\left(\mathcal{K}^{\prime}\right)$ in an analogous way to that between $\mathcal{K}$ and $\mathcal{K}^{\prime}$, by replacing $\operatorname{FM}(\mathcal{K})$ and $\operatorname{FM}\left(\mathcal{K}^{\prime}\right)$ in the initial definition with $\operatorname{Rep}(\mathcal{K})$ and $\operatorname{Rep}\left(\mathcal{K}^{\prime}\right)$, respectively. Let $\operatorname{Select}_{X}\left(\mathcal{K}, \mathcal{K}^{\prime}\right)$ be the set of featured models $\mathcal{F}^{\prime}$ in $\operatorname{Rep}\left(\mathcal{K}^{\prime}\right)$ such that there exist some featured models $\mathcal{F}$ in $\operatorname{Rep}(\mathcal{K})$ and the X-distance between $\mathcal{F}$ and $\mathcal{F}^{\prime}$ is minimal between $\operatorname{Rep}(\mathcal{K})$ and $\operatorname{Rep}\left(\mathcal{K}^{\prime}\right)$.

Proposition 5.5. Given a $K B \mathcal{K}$ and a signature $\mathcal{S}$, let $\left|\mathcal{S}_{R}\right|=m$ and $\left|\mathcal{S}_{I}\right|=n$. Suppose Algorithm 1 returns $\operatorname{Rep}(\mathcal{K})$, then the following conditions hold:

(1) $\operatorname{Rep}(\mathcal{K}) \subseteq \operatorname{FM}(\mathcal{K})$ and each featured model in $\operatorname{Rep}(\mathcal{K})$ has at most $4 m+2 n+2$ types. 
(2) For another $K B \mathcal{K}^{\prime}$ on $\mathcal{S}$ and $X=P$ or $S$, Select ${ }_{X}\left(\mathcal{K}, \mathcal{K}^{\prime}\right) \subseteq \operatorname{FM}\left(\mathcal{K} \circ \circ_{X} \mathcal{K}^{\prime}\right)$; and for each $\mathcal{F} \in \operatorname{FM}\left(\mathcal{K} \circ_{X} \mathcal{K}^{\prime}\right)$ and a type $\tau$ in $\mathcal{F}$, there exists $\mathcal{F}^{\prime} \in \operatorname{Select}_{X}\left(\mathcal{K}, \mathcal{K}^{\prime}\right)$ that has the same Herbrand set as $\mathcal{F}$ and contains $\tau$.

The second half of Proposition 5.5 states that while $\operatorname{Select}_{X}\left(\mathcal{K}, \mathcal{K}^{\prime}\right)$ is a subset of $\operatorname{FM}\left(\mathcal{K} \circ_{X} \mathcal{K}^{\prime}\right)$, the types and Herbrand sets occurring in $\operatorname{FM}\left(\mathcal{K} \circ_{X} \mathcal{K}^{\prime}\right)$ are contained in $\operatorname{Select}_{X}\left(\mathcal{K}, \mathcal{K}^{\prime}\right)$. This property is crucial for the computation of the MA of revision through representative featured models.

Now, we show that the MA of $\mathcal{K} \circ_{X} \mathcal{K}^{\prime}$, with $X=P$ or $X=S$, can be computed from $\operatorname{Rep}(\mathcal{K})$ and $\operatorname{Rep}\left(\mathcal{K}^{\prime}\right)$. The following algorithm (ref. Algorithm 2 constructs the MA of $\mathcal{K} \circ_{X} \mathcal{K}^{\prime}$ from $\operatorname{Select}_{X}\left(\mathcal{K}, \mathcal{K}^{\prime}\right)$. Indeed, the algorithm is applicable to any revision operator with a valid definition of $\operatorname{Select}_{X}\left(\mathcal{K}, \mathcal{K}^{\prime}\right)$, and the MA construction applies to an arbitrary set $\mathbb{F}$ of featured interpretations. For an $\mathcal{S}$-type $\tau$, denote $C o n(\tau)=\prod_{B \in \tau} B \sqcap \prod_{B \in B C_{\mathcal{S}} \backslash \tau} \neg B$.

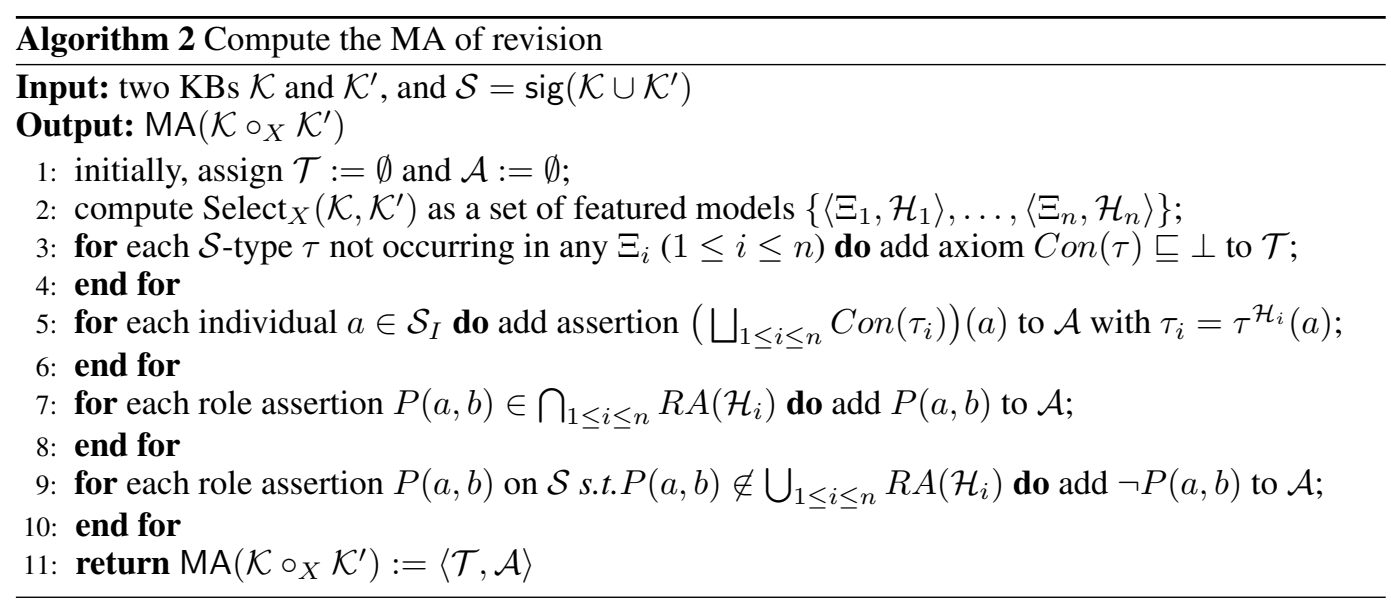

THEOREM 5.6. Given two consistent $K B s \mathcal{K}$ and $\mathcal{K}^{\prime}$, Algorithm 2 returns the $M A$ of $\mathcal{K} \circ_{X} \mathcal{K}^{\prime}$, where $X=P$ or $X=S$.

Proof Let $\mathcal{K}^{*}$ be the KB output by a slightly changed version of Algorithm 2, by replacing $\operatorname{Select}_{X}\left(\mathcal{K}, \mathcal{K}^{\prime}\right)$ with $\operatorname{FM}\left(\mathcal{K}, \mathcal{K}^{\prime}\right)$. We first show that $\mathcal{K}^{*}$ is the MA of $\mathcal{K} \circ_{X} \mathcal{K}^{\prime}$. Clearly, $\mathcal{K}^{*}$ is over $\mathcal{S}$. In the same way as the proof of Proposition 3.24, it can be verified that for each featured model $\mathcal{F}$ in $\operatorname{FM}\left(\mathcal{K} \circ \mathcal{K}^{\prime}\right), \mathcal{F}$ satisfies $\mathcal{K}^{*}$. That is, $\operatorname{FM}\left(\mathcal{K} \circ \mathcal{K}^{\prime}\right) \subseteq \mathrm{FM}\left(\mathcal{K}^{*}\right)$. We only need to show that for any $\mathrm{KB} \mathcal{K}^{\prime \prime}$ over $\mathcal{S}$ such that $\mathrm{FM}\left(\mathcal{K} \circ \mathcal{K}^{\prime}\right) \subseteq \mathrm{FM}\left(\mathcal{K}^{\prime \prime}\right), \operatorname{FM}\left(\mathcal{K}^{*}\right) \subseteq \operatorname{FM}\left(\mathcal{K}^{\prime \prime}\right)$.

Suppose $\mathcal{K}^{\prime \prime}=\left\langle\mathcal{T}^{\prime \prime}, \mathcal{A}^{\prime \prime}\right\rangle$. Since we assume each $\left\langle\Xi_{i}, \mathcal{H}_{1}\right\rangle$ in $\operatorname{Algorithm} 2$ is from $\operatorname{FM}\left(\mathcal{K}, \mathcal{K}^{\prime}\right)$, $\left\langle\Xi_{i}, \mathcal{H}_{1}\right\rangle$ satisfies $\mathcal{T}^{\prime \prime}$ and $\mathcal{A}^{\prime \prime}$ for $1 \leq i \leq n$. We want to show that each featured model $\mathcal{F}=\langle\Xi, \mathcal{H}\rangle$ of $\mathcal{K}^{*}$ also satisfies $\mathcal{T}^{\prime \prime}$ and $\mathcal{A}^{\prime \prime}$. Firstly, $\Xi \subseteq \bigcup_{1 \leq i \leq n} \Xi_{i}$. Suppose otherwise, there exists a type $\tau \in \Xi \backslash \bigcup_{1 \leq i \leq n} \Xi_{i}$. From Line 3 of Algorithm 2 , $\operatorname{Con}(\tau) \sqsubseteq \perp$ is in $\mathcal{T}$. However, $\tau$ does not satisfy $\operatorname{Con}(\tau) \sqsubseteq \perp$, and thus $\mathcal{F}$ does not satisfy $\mathcal{T}$, which is a contradiction. Now, we have shown that $\Xi \subseteq \bigcup_{1 \leq i \leq n} \Xi_{i}$. Since each type in $\Xi_{i}$ satisfies $\mathcal{T}^{\prime \prime}$ for $1 \leq i \leq n$, all the types in $\Xi$ satisfy $\mathcal{T}^{\prime \prime}$. That is, $\mathcal{\mathcal { F }}$ satisfies $\mathcal{T}^{\prime \prime}$. Secondly, for each concept assertion $C(a)$ in $\mathcal{A}^{\prime \prime}$, we want to show that $\mathcal{F}$ satisfies $C(a)$. In Line 5, $\left(\bigsqcup_{1 \leq i \leq n} \operatorname{Con}\left(\tau_{i}\right)\right)(a)$ is added into $\mathcal{A}$, and thus $\tau^{\mathcal{H}}(a)$ must satisfy concept $\bigsqcup_{1 \leq i \leq n} \operatorname{Con}\left(\tau_{i}\right)$. Then, $\tau^{\mathcal{H}}(a)=\tau_{i}$ must hold for some $1 \leq i \leq n$, as otherwise $\tau^{\mathcal{H}}(a)$ would satisfy $\neg\left(\bigsqcup_{1 \leq i \leq n} \operatorname{Con}\left(\tau_{i}\right)\right)$. That is, $\tau^{\mathcal{H}}(a)=\tau^{\mathcal{H}_{i}}(a)$. Since $\mathcal{F}_{i}$ satisfies $C(a)$ in $\mathcal{A}^{\prime \prime}$ and thus $\tau^{\mathcal{H}_{i}}(a)$ satisfies $C, \mathcal{F}$ also satisfies $C(a)$. Finally, for each role assertion $R(a, b)$ in $\mathcal{A}^{\prime \prime}$, we want to show that $\mathcal{F}$ satisfies $R(a, b)$. Since $\mathcal{F}_{i}$ satisfies $R(a, b), R(a, b) \in \mathcal{H}_{i}$ for $1 \leq i \leq n . R(a, b)$ is added to $\mathcal{A}$ in Line 7, and thus, $\mathcal{F}$ satisfies $R(a, b)$. Similarly, for each role assertion $\neg R(a, b)$ in 
$\mathcal{A}^{\prime \prime}, P(a, b) \notin \mathcal{H}_{i}$ for $1 \leq i \leq n$ and $\neg R(a, b)$ is added to $\mathcal{A}$ in Line 9 . Thus, $\mathcal{F}$ satisfies $\neg P(a, b)$. We have shown that $\mathcal{F}$ satisfies both $\mathcal{T}^{\prime \prime}$ and $\mathcal{A}^{\prime \prime}$. Thus, $\mathrm{FM}\left(\mathcal{K}^{*}\right) \subseteq \mathrm{FM}\left(\mathcal{K}^{\prime \prime}\right)$.

Now, by replacing $\operatorname{FM}\left(\mathcal{K}, \mathcal{K}^{\prime}\right)$ back with $\operatorname{Select}_{X}\left(\mathcal{K}, \mathcal{K}^{\prime}\right)$, we want to show that $\mathcal{K}^{*}$ is still returned by Algorithm 2 . From Proposition 5.5. $\operatorname{Select}_{X}\left(\mathcal{K}, \mathcal{K}^{\prime}\right) \subseteq \operatorname{FM}\left(\mathcal{K}, \mathcal{K}^{\prime}\right)$. Suppose $\mathrm{FM}\left(\mathcal{K}, \mathcal{K}^{\prime}\right) \backslash$ $\operatorname{Select}_{X}\left(\mathcal{K}, \mathcal{K}^{\prime}\right)=\left\{\left\langle\Xi_{j}^{\prime}, \mathcal{H}_{j}^{\prime}\right\rangle \mid 1 \leq j \leq m\right\}$. By Proposition 5.5. each $\mathcal{H}_{j}^{\prime}(1 \leq j \leq m)$ equals some $\mathcal{H}_{i}(1 \leq i \leq n)$ in $\operatorname{Select}_{X}\left(\mathcal{K}, \mathcal{K}^{\prime}\right)$. Hence, omitting $\mathcal{H}_{j}^{\prime}$ 's does not affect Lines 5-10, and hence does not affect the output. For each $\Xi_{j}^{\prime}(1 \leq j \leq m)$ and each type $\tau \in \Xi_{j}^{\prime}$, again by Proposition 5.5. $\tau \in \Xi_{i}$ for some $1 \leq i \leq n$. That is, $\bigcup_{1 \leq j \leq m} \Xi_{j}^{\prime} \subseteq \bigcup_{1 \leq i \leq n} \Xi_{i}$. Again, $\Xi_{j}^{\prime}$ 's does not affect Lines 3-4, and hence does not affect the output. We have shown that $\mathcal{K}^{*}$ is the output of Algorithm 2

Algorithm 2 runs in (single) exponential time with respect to the size of the initial KBs, and the result of revision may have an exponential blow up. It is not surprising, as even in the case of propositional logic, the size of the revision (under Satoh's revision operator in particular) is not polynomial in the size of the input, unless the polynomial hierarchy collapses [Cadoli et al. 1999]. While some other optimization techniques have been developed in a prototype implementation [Cobby et al. 2011] as a plug-in of Protégé, the algorithm is still not very efficient at this moment and thus one future issue would be developing more efficient algorithms for our revision operators.

\section{RELATED WORK}

A number of approaches to DL ontology revision have been proposed in the literature and these specific revision operators can be roughly classified into two categories: syntax-based and model-based revision approaches. According to the restrictions on the initial and new knowledge considered, these approaches can also be classified in a different dimension: TBox revisions (i.e., ABoxes are empty in $\mathcal{K}$ and $\mathcal{K}^{\prime}$ ), ABox revisions (i.e., $\mathcal{K}$ has an empty or fixed TBox and $\mathcal{K}^{\prime}$ has an empty TBox), and general $\mathrm{KB}$ revisions (i.e., both $\mathcal{K}$ and $\mathcal{K}^{\prime}$ may contain non-empty TBoxes and ABoxes). Compared to some existing ontology revision approaches that are close to our approach, our revision operators are the only ones that enjoy all of the following three properties:

- It allows the revision of a KB by another KB (with both a TBox and an ABox).

- It has a model-theoretic justification of minimal change, and preserves implicit knowledge at a degree.

— The result of revision is unique (up to semantic equivalence).

A syntax-based revision usually attempts to revise $\mathcal{K}$ with $\mathcal{K}^{\prime}$ by removing a minimal set of axioms from $\mathcal{K}$ that are responsible for the contradiction to $\mathcal{K}^{\prime}$, before adding $\mathcal{K}^{\prime}$. This idea was first briefly discussed for DL KB revision in [Haase and Stojanovic 2005] and then further pursued in [Qi et al. 2008] which focuses on TBox revision and on resolving incoherence (instead of inconsistency). A similar idea is used to study (non-AGM) rationality postulates and representation theorems for KB revision in [Ribeiro and Wassermann 2009]. The definition of a syntax-based operator for ontology revision is usually coupled with a relatively efficient algorithm for computing revision, and it can preserve the original syntactic structure of the initial $\mathrm{KB}$, which is useful for some practical applications. On the other hand, most syntax-based approaches to ontology revision lack of a suitable semantic justification, that is, there is no semantic measure for the closeness between the initial ontology and the result of revision (a new ontology). This is partially reflected in their inability of preserving any implicit knowledge. For example, given $\mathcal{K}=\{$ Student $\sqsubseteq$ Person, Student(John) $\}$, the revision of $\mathcal{K}$ by $\mathcal{K}^{\prime}=\{\neg$ Student (John) $\}$ by syntax-based approaches in [Haase and Stojanovic 2005: Ribeiro and Wassermann 2009] will discard implicit information Person(John), although it is not a source of the contradiction. On the other hand, both of our revision operators preserve Person(John). Also, Example 4.11 and the example in Section 4.4 show that our revision can revise axioms, whereas syntax-based ones like [Haase and Stojanovic 2005; Ribeiro and Wassermann 2009] cannot. 
It is argued in [Cuenca Grau et al. 2012] that a revision operator should (maximally) preserve implicit knowledge in the initial KB $\mathcal{K}$. In order to achieve this, some more recent works, such as [Calvanese et al. 2010; Cuenca Grau et al. 2012], advocate to define syntax-based ontology revision on the deductive closure $c l(\mathcal{K})$ of $\mathcal{K}$ instead of $\mathcal{K}$ itself. This of course relies on the finiteness of the deductive closure, with the exception of some special cases (e.g., the contraction operator in [Cuenca Grau et al. 2012] for a fragment of $\mathcal{E} \mathcal{L}$ that admits infinite deductive closures). However, another issue remains, that is, there may not exist a unique optimal solution to syntax-based revision [Calvanese et al. 2010], since often a number of minimal sets of axioms in $\mathcal{K}($ or $c l(\mathcal{K})$ ) exist that are responsible for the contradictions. For instance in Example 4.2, a syntax-based revision operator like those in [Calvanese et al. 2010; Cuenca Grau et al. 2012] have at least three options: removing PhDStudent $\sqsubseteq$ Student, removing Student $\sqsubseteq \neg$ JhasStafflD, and removing PhDStudent(John), respectively, resulting in at least three results of revision. Although a few special cases of DL-Lite ABox revision admit unique optimal solutions [Calvanese et al. 2010, Lenzerini and Savo 2011], these approaches either do not address KB revision [Lenzerini and Savo 2011], or exhibit nondeterministic behaviours when handling KBs [Calvanese et al. 2010; Cuenca Grau et al. 2012]. In contrast to most syntax-based revision, the result of our revisions with MA is always unique (up to semantic equivalence).

A model-based approach addresses ontology revision and minimal change from a model-theoretic perspective: since each model of the result of revision needs to satisfy the new knowledge $\mathcal{K}^{\prime}$, a model-based operator usually collects the set $\mathcal{M}$ of models of $\mathcal{K}^{\prime}$ that are closest (w.r.t. a definition of distance) to the models of $\mathcal{K}$, and defines the result of revision to be the KB axiomatising $\mathcal{M}$. The approaches proposed in [Giacomo et al. 2009, Qi and Du 2009, Liu et al. 2011, Kharlamov et al. 2013; Qi et al. 2015] can be classified as model-based revisions. While [Qi and Du 2009] follows the classical belief revision approach, where closeness is measured w.r.t. the whole set of models of $\mathcal{K}$, [Giacomo et al. 2009, Liu et al. 2011] adopt the classical belief update approach and measure closeness w.r.t. individual models of $\mathcal{K}$. Moreover, [Kharlamov et al. 2013] studies both ontology revision and update, and shows that both approaches coincide when revising ABoxes in a fragment of DL-Lite and under a certain notion of distance. In this paper, we focus on the belief revision approach.

A model-based revision provides a natural semantic justification for minimal change (through model distance) and the result of revision is uniquely defined (up to semantic equivalence). Two important yet challenging questions for these approaches are how to address the inexpressibility issue and how to compute (or approximate) the result of revision [Kharlamov et al. 2013]. The existing approaches have answered the two questions only for some restricted cases. Approaches to ABox revision are proposed in [Giacomo et al. 2009, Liu et al. 2011; Kharlamov et al. 2013, Qi et al. 2015], and a proposal for TBox revision is provided in [Qi and Du 2009]. It is worth mentioning that simply combining TBox revisons and ABox revisions cannot handle the general KB revision task - for example, it cannot handle the case where $\mathcal{K}$ contains a non-empty ABox and $\mathcal{K}^{\prime}$ contains a non-empty TBox. In this paper, the $\mathrm{P}^{*}$-revision is defined in a similar way as [Qi and Du 2009] and the symbol-based revision in [Kharlamov et al. 2013], but for general KBs. Corollary 4.8 connects our P-revision and the model-based $\mathrm{P}^{*}$-revision. While Example 4.9 shows that these two do not coincide in general, they have the same MA. Hence, through our algorithm for computing the MA of P-revision, we provide to the best of our knowledge, the first algorithm to compute the MA of model-based revision for general KBs.

Besides defining specific revision operators, there have been some efforts to capture ontology revision by adapting the AGM postulates [Alchourrón et al. 1985]. In [Flouris et al. 2005], the authors adapt the AGM postulates for belief contraction to DLs and study conditions for a contraction operator defined in DLs to be compliant with these postulates. It is shown that for many DLs including DL-Lite, a result of AGM-compliant contraction may not be expressible in the same DLs. While in propositional logic, belief revision can be defined via belief contraction [Alchourrón et al. 1985], it is not straightforward to define ontology revision operators through contraction, largely due to the lacking of a well-defined notion of axiom negation. While [Flouris et al. 2006] attempts to address 
this issue, no concrete revision operator is defined. A set of adapted AGM postulates for ontology revision is introduced in [Qi et al. 2006], but the proposed revision operator is syntactic and thus only satisfies a weakened version of the postulates. The AGM theory has also been studied in general logics that cover most DLs [Ribeiro et al. 2013; Zhuang et al. 2015], yet no concrete operators were provided.

Under different assumptions and settings, some other actively studied but loosely related approaches include ontology debugging, repair, interactive revision, action formalisms, and inconsistency-tolerant reasoning. Ontology debugging and repair methods [Kalyanpur et al. 2005, Schlobach et al. 2007] are able to pin down the sources of contradictions, and can be used as supporting tools for ontology revision. Yet these approaches themselves do not provide a revision mechanism. An interactive revision tool [Nikitina et al. 2012] can assist human users in evaluating the plausibility of axioms, and the ontology can then be revised by the users via approving or declining the evaluated axioms. Such a revision process is not fully automated and the users are not assisted in correcting/refining axioms. DL-based action formalisms [Baader et al. 2005;, Baader] et al. 2010] use ABoxes to describe states of the world (before and after actions) and TBoxes to describe background knowledge. These proposals concern the use of ontologies in formalising actions, rather than the change of ontologies. Finally, inconsistency-tolerant approaches [Rosati et al. 2012, Lukasiewicz et al. 2012, Bienvenu and Rosati 2013] handle inconsistency by reasoning over repairs of an inconsistent ontology but do not make changes to the ontology.

\section{CONCLUSION}

Motivated by the emerging need in ontology engineering and maintenance and the lack of sufficient tool support, our work aims to shed new light to the global effort of developing automated ontology change mechanisms. To provide a balanced approach between the extant syntax-based and modelbased approaches, which both have their advantages and limitations, we have introduced the notion of featured interpretations, based on which an alternative semantics for DL-Lite is defined. The structure of a featured interpretation extends that of a classical Herbrand model and that of a DLLite type, by allowing a Herbrand set to capture the ABox and a set of types to capture the TBox. Unlike classical models of DLs, each featured model is a finite structure and the number of featured models for a DL-Lite KB is also finite. Moreover, we have shown that the semantics determined by featured models coincides with classical semantics with respect to almost all major reasoning tasks for DL-Lite. Also, we studied the expressibility of featured interpretations; that is, whether and when a set of featured interpretations can be axiomatised via a DL-Lite KB. As a result, our new semantics is another step towards a balanced approach to ontology change, by potentially providing a unified framework for developing ontology change methods including revision, contraction, update, merging, and forgetting.

We have demonstrated how to use our new semantics to cast propositional belief revision techniques to handle general DL-Lite ontology revision tasks (where the initial KB and the new KB both consist of TBoxes and ABoxes). In particular, we have defined two specific operators for DLLite $_{\text {bool }}^{\mathcal{N}}$, based on two notions of distance: one captures the intuition of a classical model distance and the other is more fine-grained. The rationality of our revision operators are justified by adapted AGM postulates, and their applicability is shown via a case study. As the result of revision is in general a disjunctive $\mathrm{KB}$, we studied the maximal approximation of revision (as a single $\mathrm{KB}$ ) in DL-Lite. The approximated KB is sufficient in practice as it coincides with the result of revision whenever it is expressible; and the approximated KB behaves exactly as the result of revision with respect to almost all major reasoning tasks for DL-Lite. We have developed algorithms to compute maximal approximations of ontology revisions, which would be far from straightforward if our approach were based on DL models. An important result of this paper is that the complexity of our revision operators is on the same level as major revision operators in propositional logic. We note that other propositional change operators, for example Dalal's revision, belief contraction and update can also be adapted to DL-Lite based on our semantics in a similar manner. 
There are several interesting issues that we are currently working on or that remains for future work. First, while the new semantics provides a finite semantic characterisation for DL-Lite, which is a big step from classical models to more compact semantic characterisations, the structure of featured interpretations can be further simplified in special cases. In particular, if we confine ourselves to only TBoxes or only ABoxes change, then type-based and Herbrand set-based semantics will be sufficient, respectively. However, the definition of a type-based semantics is non-trivial given the issues shown in Example 3.9. Some continuing research on this direction shows promising results [Zhuang et al. 2014, Wang et al. 2015]. Second, although the definition of featured interpretations can be applied to more expressive DLs that allow unbounded nesting of quantifications (with the definition featured models also adapted), the corresponding semantics will be less faithful in capturing the classical semantics of the corresponding DLs. Thus, it would be interesting to extend our semantic characterisation to other DLs. Instead of tweaking an individual DL, it would be an interesting research topic to develop a characterisation for a (relatively large) class of DLs. This is challenging as although types have been defined for more expressive DLs [Ghilardi et al. 2006], the structure of a type is very complex. Third, the implementation of our revision operators is still not scalable, which is not surprising given the complexity of revision even in propositional case. However, given that the current implementation is still at a preliminary stage, there is a lot of space for optimisations. Some recent progress include [Qi et al. 2015], where graph database techniques are employed to develop an efficient ABox revision system. Fourth, we plan to study some other properties of our revision operators to better understand their relations with classical belief revision. For instance, it would be interesting to develop a presentation theorem for $\mathrm{KB}$ revision similar as the one for TBox revision [Zhuang et al. 2014]. Finally, we are working on merging and para-consistency reasoning for DL-Lite KBs.

\section{Acknowledgement}

We would like to thank the three anonymous referees whose comments helped significantly improve the quality of the paper. This work was partially supported by Australian Research Council (ARC) under grants DP110101042 and DP130102302.

\section{REFERENCES}

C. Alchourrón, P. Gärdenfors, and D. Makinson. 1985. On the Logic of Theory Change: Partial Meet Contraction and Revision Functions. Journal of Symbolic Logic 50 (1985), 510-530.

A. Artale, D. Calvanese, R. Kontchakov, and M. Zakharyaschev. 2009. The DL-Lite Family and Relations. Journal of Artificial Intelligence Research 36 (2009), 1-69.

F. Baader, D. Calvanese, D. L. McGuinness, D. Nardi, and P. F. Patel-Schneider. 2003. The Description Logic Handbook. Cambridge University Press.

F. Baader, M.1 Lippmann, and H. Liu. 2010. Using Causal Relationships to Deal with the Ramification Problem in Action Formalisms Based on Description Logics. In Proceedings of the 17th International Conference on Logic for Programming, Artificial Intelligence, and Reasoning (LPAR-17). 82-96.

F. Baader, C. Lutz, M. Milicic, U. Sattler, and F. Wolter. 2005. Integrating Description Logics and Action Formalisms: First Results. In Proceedings of the 20th National Conference on Artificial Intelligence (AAAI-05). 572-577.

M. Bienvenu and R. Rosati. 2013. Tractable Approximations of Consistent Query Answering for Robust Ontology-based Data Access. In Proceedings of the 23rd International Joint Conference on Artificial Intelligence (IJCAI-13).

M. Cadoli, F. M. Donini, P. Liberatore, and M. Schaerf. 1999. The Size of a Revised Knowledge Base. Artificial Intelligence 115,1 (1999), 25-64.

D. Calvanese, G. De Giacomo, D. Lembo, M. Lenzerini, and R. Rosati. 2006. Data Complexity of Query Answering in Description Logics. In Proceedings of the 10th International Conference on Principles of Knowledge Representation and Reasoning (KR-06). 260-270.

D. Calvanese, G. De Giacomo, D. Lembo, M. Lenzerini, and R. Rosati. 2007. Tractable Reasoning and Efficient Query Answering in Description Logics: The DL-Lite Family. Journal of Automatic Reasoning 39, 3 (2007), 385-429.

D. Calvanese, E. Kharlamov, W. Nutt, and D. Zheleznyakov. 2010. Evolution of DL-Lite Knowledge Bases. In Proceedings of the 9th International Semantic Web Conference (ISWC-10). 112-128.

N. Cobby, K. Wang, Z. Wang, and M. Sotomayor. 2011. OntoRevision: A Plug-in System for Ontology Revision in Protégé. In Proceedings of the Joint International Semantic Technology Conference (JIST-11). 417-424. 
N. Creignou, O. Papini, R. Pichler, and S. Woltran. 2012. Belief Revision within Fragments of Propositional Logic. In Proceedings of the 13th International Conference on Principles of Knowledge Representation and Reasoning (KR-12). 126-136.

B. Cuenca Grau, I. Horrocks, Y. Kazakov, and U. Sattler. 2008. Modular Reuse of Ontologies: Theory and Practice. Journal of Artificial Intelligence Research 31 (2008), 273-318.

B. Cuenca Grau, E. Jimenez Ruiz, E. Kharlamov, and D. Zhelenyakov. 2012. Ontology Evolution under Semantic Constraints. In Proceedings of the 13th International Conference on Principles of Knowledge Representation and Reasoning (KR-12). 137-147.

M. Dalal. 1988. Investigations into a Theory of Knowledge Base Revision. In Proceedings of the 7th National Conference on Artificial Intelligence (AAAI-88). 475-479.

T. Eiter and G. Gottlob. 1992. On the Complexity of Propositional Knowledge Base Revision, Updates, and Counterfactuals. Artificial Intelligence 57 (1992), 227-270.

G. Flouris, Z. Huang, J. Z. Pan, D. Plexousakis, and H. Wache. 2006. Inconsistencies, Negations and Changes in Ontologies. In Proceedings of the 21st National Conference on Artificial Intelligence (AAAI-06). 1295-1300.

G. Flouris, D. Manakanatas, H. Kondylakis, D. Plexousakis, and G. Antoniou. 2008. Ontology Change: Classification and Survey. The Knowledge Engineering Review 23, 2 (2008), 117-152.

G. Flouris, D. Plexousakis, and G. Antoniou. 2005. On Applying the AGM Theory to DLs and OWL. In Proceedings of the 4th International Semantic Web Conference (ISWC-05). 216-231.

S. Ghilardi, C. Lutz, and F. Wolter. 2006. Did I Damage My Ontology? A Case for Conservative Extensions in Description Logics. In Proceedings of the 10th International Conference on Principles of Knowledge Representation and Reasoning (KR-06). 187-197.

G. De Giacomo, M. Lenzerini, A. Poggi, and R. Rosati. 2007. On the Approximation of Instance Level Update and Erasure in Description Logics. In Proceedings of the 22th National Conference on Artificial Intelligence (AAAI-07). 403-408.

G. De Giacomo, M. Lenzerini, A. Poggi, and R. Rosati. 2009. On Instance-level Update and Erasure in Description Logic Ontologies. Journal of Logic and Computation 19, 5 (2009), 745-770.

P. Haase and L. Stojanovic. 2005. Consistent Evolution of OWL Ontologies. In Proceedings of the 2nd European Semantic Web Conference (ESWC-05). 182-197.

F. W. Hartel, S. de Coronado, R. Dionne, G. Fragoso, and J. Golbeck. 2005. Modeling A Description Logic Vocabulary for Cancer Research. Journal of Biomedical Informatics 38, 2 (2005), 114-129.

M. Horridge, B. Parsia, and U. Sattler. 2009. Explaining Inconsistencies in OWL Ontologies. In Proceedings of the 3rd International Conference on Scalable Uncertainty Managemen (SUM-09). 124-137.

A. Kalyanpur, B. Parsia, E. Sirin, and J. A. Hendler. 2005. Debugging Unsatisfiable Classes in OWL Ontologies. Journal of Web Semantics 3, 4 (2005), 268-293.

H. Katsuno and A. Mendelzon. 1991. Propositional Knowledge Base Revision and Minimal Change. Artificial Intelligence 52, 3 (1991), 263-294.

E. Kharlamov, D. Zheleznyakov, and D. Calvanese. 2013. Capturing Model-based Ontology Evolution at the Instance Level: The Case of DL-Lite. J. Comput. System Sci. 79, 6 (2013), 835-872.

B. Konev, R. Kontchakov, M. Ludwig, T. Schneider, F. Wolter, and M. Zakharyaschev. 2011. Conjunctive Query Inseparability of OWL 2 QL TBoxes. In Proceedings of the 25th AAAI Conference on Artificial Intelligence, (AAAI-11).

R. Kontchakov, F. Wolter, and M. Zakharyaschev. 2008. Can You Tell the Difference Between DL-Lite Ontologies?. In Proceedings of the 11th International Conference on Principles of Knowledge Representation and Reasoning (KR-08). 285-295.

R. Kontchakov, F. Wolter, and M. Zakharyaschev. 2010. Logic-based Ontology Comparison and Module Extraction, with an Application to DL-Lite. Artificial Intelligence 174, 15 (2010), 1093-1141.

M. Lenzerini and D. F. Savo. 2011. On the Evolution of the Instance Level of DL-Lite Knowledge Bases. In Proceedings of the 24th International Workshop on Description Logics (DL-11).

H. Liu, C. Lutz, M. Milicic, and F. Wolter. 2011. Foundations of Instance Level Updates in Expressive Description Logics. Artificial Intelligence 175, 18 (2011), 2170-2197.

T. Lukasiewicz, M. V. Martinez, and G. I. Simari. 2012. Inconsistency Handling in Datalog+/- Ontologies. In proceedings of the 20th European Conference on Artificial Intelligence (ECAI-12). 558-563.

C. Lutz and F. Wolter. 2011. Foundations for Uniform Interpolation and Forgetting in Expressive Description Logics. In Proceedings of the 22nd International Joint Conference on Artificial Intelligence (IJCAI-11). 989-995.

T. Meyer, K. Lee, and R. Booth. 2005. Knowledge Integration for Description Logics. In Proceedings of the 20th National Conference on Artificial Intelligence (AAAI-05). 645-650.

N. Nikitina, S. Rudolph, and B. Glimm. 2012. Interactive Ontology RSevision. Journal of Web Semantics 12 (2012), 118130. 
A. G. Pérez, M. F. López, and O. C. Garcia. 2003. Ontological Engineering with examples from the areas of Knowledge Management, e-Commerce and the Semantic Web. Springer-Verlag New York, Inc.

G. Qi and J. Du. 2009. Model-based Revision Operators for Terminologies in Description Logics. In Proceedings of the 21st International Joint Conference on Artificial Intelligence (IJCAI-09). 891-897.

G. Qi, P. Haase, Z. Huang, Q. Ji, J. Z. Pan, and J. Völker. 2008. A Kernel Revision Operator for Terminologies - Algorithms and Evaluation. In Proceedings of the 7th International Semantic Web Conference (ISWC-08). 419-434.

G. Qi, W. Liu, and D. A. Bell. 2006. Knowledge Base Revision in Description Logics. In Proceedings of 10th European Conference on Logics in Artificial Intelligence (JELIA-06). 386-398.

G. Qi, Z. Wang, K. Wang, X. Fu, and Z. Zhuang. 2015. Approximating Model-based ABox Revision in DL-Lite: Theory and Practice. In Proceedings of the 29th AAAI Conference on Artificial Intelligence (AAAI-15). 254-260.

G. Qi and F. Yang. 2008. A Survey of Revision Approaches in Description Logics. In Proceedings of the 2nd International Conference of Web Reasoning and Rule Systems (RR-08). 74-88.

M. M. Ribeiro and R. Wassermann. 2009. Base Revision for Ontology Debugging. Journal of Logic Computation 19, 5 (2009), 721-743.

M. M. Ribeiro, R. Wassermann, G. Flouris, and G. Antoniou. 2013. Minimal Change: Relevance and Recovery Revisited. Artificial Intelligence 201 (2013), 59-80.

R. Rosati, M. Ruzzi, M. Graziosi, and G. Masotti. 2012. Evaluation of Techniques for Inconsistency Handling in OWL 2 QL Ontologies. In Proceedings of the 11th International Semantic Web Conference (ISWC-12). 337-349.

K. Satoh. 1988. Nonmonotonic Reasoning by Minimal Belief Revision. In Proceedings of the International Conference on Fifth Generation Computer Systems. 455-462.

S. Schlobach, Z. Huang, R. Cornet, and F. van Harmelen. 2007. Debugging Incoherent Terminologies. Journal of Automated Reasoning 39, 3 (2007), 317-349.

S. Staab and R. Studer (Eds.). 2009. Handbook on Ontologies (2. ed.). Springer-Verlag.

Z. Wang, K. Wang, and R. Topor. 2010a. A New Approach to Knowledge Base Revision in DL-Lite. In Proceedings of the 24th National Conference on Artificial Intelligence (AAAI-10). 369-374.

Z. Wang, K. Wang, and R. Topor. 2010b. Revising General Knowledge Bases in Description Logics. In Proceedings of the 12th International Conference on Principles of Knowledge Representation and Reasoning (KR-10). 599-601.

Z. Wang, K. Wang, R. Topor, and J. Z. Pan. 2010c. Forgetting for Knowledge BSases in DL-Lite. Annals of Mathematics and Artificial Intelligence 58, 1-2 (2010), 117-151.

Z. Wang, K. Wang, Z. Zhuang, and G. Qi. 2015. Instance-driven Ontology Evolution in DL-Lite. In Proceedings of the 29th AAAI Conference on Artificial Intelligence (AAAI-15). 1656-1662.

D. Zheleznyakov, D. Calvanese, E. Kharlamov, and W. Nutt. 2010. Updating TBoxes in DL-Lite. In Proceedings of the 23rd International Workshop on Description Logics (DL-10).

Z. Zhuang, Z. Wang, K. Wang, and J. Delgrande. 2015. Extending AGM Contraction to Arbitrary Logics. In Proceedings of the 24th International Joint Conference on Artificial Intelligence (IJCAI-15). (to appear).

Z. Zhuang, Z. Wang, K. Wang, and G. Qi. 2014. Contraction and Revision over DL-Lite TBoxes. In Proceedings of the 28th AAAI Conference on Artificial Intelligence (AAAI-14). 1149-1156. 


\section{A. PROOFS FOR SECTION 3}

Lemma 3.3 Given an interpretation $\mathcal{I}$ over $\mathcal{S}$, for $d_{1}, d_{2} \in \Delta^{\mathcal{I}}$, if $d_{1}$ and $d_{2}$ are type-equivalent, then $d_{1} \in C^{\mathcal{I}}$ iff $d_{2} \in C^{\mathcal{I}}$ for each concept $C$ over $\mathcal{S}$.

Proof We show the lemma by induction.

If $C$ is a basic concept, then $C \in \tau^{\mathcal{I}}\left(d_{1}\right)$ iff $C \in \tau^{\mathcal{I}}\left(d_{2}\right)$. Thus, $d_{1} \in C^{\mathcal{I}}$ iff $d_{2} \in C^{\mathcal{I}}$.

Assume that the conclusion is true for concepts $C_{1}$ and $C_{2}$, and $C$ is constructed from $C_{1}$ and $C_{2}$ in the following two ways:

Case 1. $C=\neg C_{1}$ : By the induction hypothesis, $d_{1} \in\left(C_{1}\right)^{\mathcal{I}}$ iff $d_{2} \in\left(C_{1}\right)^{\mathcal{I}}$. Thus, $d_{1} \in C^{\mathcal{I}}$ iff $d_{1} \notin\left(C_{1}\right)^{\mathcal{I}}$ iff $d_{2} \notin\left(C_{1}\right)^{\mathcal{I}}$ iff $d_{2} \in C^{\mathcal{I}}$.

Case 2. $C=C_{1} \sqcap C_{2}: d_{1} \in C^{\mathcal{I}}$ iff $d_{1} \in C_{1}^{\mathcal{I}}$ and $d_{1} \in C_{2}^{\mathcal{I}}$. By the induction hypothesis, $d_{1} \in C_{i}^{\mathcal{I}}$ iff $d_{2} \in C_{i}^{\mathcal{I}}$ for $i=1,2$. Thus, $d_{1} \in C^{\mathcal{I}}$ iff $d_{2} \in C_{i}^{\mathcal{I}}$.

Lemma 3.4 Let $C$ be any concept over $\mathcal{S}$. Given an interpretation $\mathcal{I}$ over $\mathcal{S}, d \in C^{\mathcal{I}}$ iff $\tau^{\mathcal{I}}(d)$ satisfies $C$.

Proof We show the lemma by induction.

If $C$ is a basic concept, then $d \in C^{\mathcal{I}}$ iff $C \in \tau^{\mathcal{I}}(d)$.

Assume that the conclusion is true for concepts $C_{1}$ and $C_{2}$, and $C$ is constructed from $C_{1}$ and $C_{2}$ in the following two ways:

Case 1. $C=\neg C_{1}$ : By the induction hypothesis, $d \in\left(C_{1}\right)^{\mathcal{I}}$ iff $\tau^{\mathcal{I}}(d)$ satisfies $C_{1}$. Thus, $d \in C^{\mathcal{I}}$ iff $d \in\left(\neg C_{1}\right)^{\mathcal{I}}$ iff $d \notin\left(C_{1}\right)^{\mathcal{I}}$ iff $\tau^{\mathcal{I}}(d)$ does not satisfy $C_{1}$ iff $\tau^{\mathcal{I}}(d)$ satisfies $\neg C_{1}$.

Case 2. $C=C_{1} \sqcap C_{2}: d \in C^{\mathcal{I}}$ iff $d \in C_{1}^{\mathcal{I}}$ and $d \in C_{2}^{\mathcal{I}}$. By the induction hypothesis, $d \in C_{i}^{\mathcal{I}}$ iff $\tau^{\mathcal{I}}(d)$ satisfies $C_{i}$ for $i=1,2$. Thus, $d \in C^{\mathcal{I}}$ iff $\tau^{\mathcal{I}}(d)$ satisfies $C$.

Proposition 3.5 Let $C$ be a concept and $\mathcal{T}$ be a role coherent TBox over $\mathcal{S}$. $C$ is satisfiable w.r.t. $\mathcal{T}$ iff $C$ is type-satisfiable w.r.t. $\mathcal{T}$.

Proof For the "only if" direction, suppose $C$ is satisfiable w.r.t. $\mathcal{T}$, then there exists a model $\mathcal{I}$ of $\mathcal{T}$ and $d \in \Delta^{\mathcal{I}}$ such that $d \in C^{\mathcal{I}}$. Note that we also have $d \in\left(\neg C_{1} \sqcup C_{2}\right)^{\mathcal{I}}$ for each $C_{1} \sqsubseteq C_{2} \in \mathcal{T}$, as $\mathcal{T} \models \top \sqsubseteq \neg C_{1} \sqcup C_{2}$. By Lemma 3.4. $\tau^{\mathcal{I}}(d)$ satisfies $C$. Also, $\tau^{\mathcal{I}}(d)$ satisfies $\neg C_{1} \sqcup \bar{C}_{2}$ for each $C_{1} \sqsubseteq C_{2} \in \mathcal{T}$. That is, $\tau^{\mathcal{I}}(d)$ satisfies both $\mathcal{T}$ and $C$.

For the "if" direction, assume that there exists a type $\tau_{0}$ satisfying both $\mathcal{T}$ and $C$. Let $\Xi$ be the set of all types satisfying $\mathcal{T}$. For each $\tau \in \Xi$ and $R \in \mathcal{S}_{R} \cup \mathcal{S}_{R}^{-}$, we define $\operatorname{suc}_{R}(\tau)$ as follows: if $\exists R \notin \tau$, let $s u c_{R}(\tau)=0$; otherwise, let $s u c_{R}(\tau)=n$ for the maximal $n \geq 1$ such that $\geqslant n R \in \tau$.

We construct a tree-shaped interpretation $T$ from $\tau_{0}$ and $\Xi$, where the nodes of $T$ are domain elements. Each node is labelled with a type in $\Xi$, and each edge is labelled with a role in $\mathcal{S}_{R} \cup \mathcal{S}_{R}^{-}$. We say a node is a $R$-child of its parent node if the edge between them has a label $R$. Moreover, we require that:

— the root node $d_{0}$ is labelled with $\tau_{0}$;

— for each node $d$ labelled with $\tau$ and each role $R \in \mathcal{S}_{R} \cup \mathcal{S}_{R}^{-}, d$ has $n R$-children $d_{i}(i=1, \ldots, n)$, where $n=\operatorname{suc}_{R}(\tau)-1$ if $d$ is an $R^{-}$-child itself, and otherwise, $n=s u c_{R}(\tau)$; and

- each $R$-child $d_{i}$ is labelled with a type $\tau_{i}$ such that $s u c_{R^{-}}\left(\tau_{i}\right)>0$.

To show that we can construct a (possibly infinite) tree $T$ satisfying the above three conditions, note that meeting the first condition is straightforward. The third condition requires a type $\tau_{i}$ containing $\exists R^{-}$in $\Xi$ to label each $R$-child. Since $\mathcal{T}$ is role coherent, for each role name $P$ in $\mathcal{T}$, both $\exists P$ and $\exists P^{-}$are satisfiable in $\mathcal{T}$. From the first part of the proof, there exist types $\tau_{P}$ and $\tau_{P^{-}}$in $\Xi$ containing $\exists P$ and $\exists P^{-}$respectively. That is, $\operatorname{suc}_{P}\left(\tau_{P}\right)>0$ and $\operatorname{suc}_{P^{-}}\left(\tau_{P^{-}}\right)>0$. Hence, we can take $\tau_{i}=\tau_{R^{-}}$from $\Xi$. For the second condition to be satisfied, we only need to make sure $n \geq 0$. It is clear if $d$ is not an $R^{-}$-child; when $d$ is an $R^{-}$-child, by the third condition, we have $\operatorname{suc}_{R}(\tau)>0$ and thus $n=\operatorname{suc}_{R}(\tau)-1 \geq 0$.

We define interpretation $\mathcal{I}$ from $T$ in an obvious way:

— Let $\Delta^{\mathcal{I}}$ be the set of the nodes in $T$. 
— For each $A \in \mathcal{S}_{C}$, let $A^{\mathcal{I}}=\{d \mid d$ has label $\tau$ containing $A\}$.

- For each $P \in \mathcal{S}_{R}$, let $P^{\mathcal{I}}=\left\{(d, e) \mid(d, e)\right.$ has label $P$ or $(e, d)$ has label $\left.P^{-}\right\}$.

Now, we need to show that $\mathcal{I}$ is a model of $\mathcal{T}$ satisfying $C$. For each $d \in \Delta^{\mathcal{I}}$, from the definition of $\mathcal{I}, \tau^{\mathcal{I}}(d)$ is exactly the label of $d$. As $\tau^{\mathcal{I}}(d)$ satisfies $\mathcal{T}$, from Lemma 3.4. $d \in\left(\neg C_{1} \sqcup C_{2}\right)^{\mathcal{I}}$ for each $C_{1} \sqsubseteq C_{2} \in \mathcal{T}$. That is, $\mathcal{I}$ is a model of $\mathcal{T}$. Moreover, as $\tau_{0}$ satisfies $C$, from Lemma 3.4, $d_{0} \in C^{\mathcal{I}}$. That is, $C$ is satisfiable w.r.t. $\mathcal{T}$.

Proposition 3.8 Let $\mathcal{A}$ be an $A B o x, C(a)$ be a concept assertion, and $P(a, b)$ be a role assertion over $\mathcal{S}$. We have $\mathcal{A} \models C(a)$ iff $\mathcal{A}=_{h} C(a)$, and $\mathcal{A} \models P(a, b)$ iff $\mathcal{A} \models_{h} P(a, b)$.

Proof We only show the statement for $C(a)$, and that for $P(a, b)$ can be shown in the same way.

For the "if" direction, suppose that $\mathcal{A} \not \models C(a)$. Then there exists a model $\mathcal{I}$ of $\mathcal{A}$ such that $\mathcal{I}$ does not satisfy $C(a)$. Let $\mathcal{H}$ be the Herbrand set obtained from $\mathcal{I}$ as follows:

$$
\begin{aligned}
\mathcal{H}= & \left\{B(b) \mid B \in B C_{\mathcal{S}}, b \in \mathcal{S}_{I}, b^{\mathcal{I}} \in B^{\mathcal{I}}\right\} \cup \\
& \left\{P(b, c) \mid P \in \mathcal{S}_{R}, b, c \in \mathcal{S}_{I},\left(b^{\mathcal{I}}, c^{\mathcal{I}}\right) \in P^{\mathcal{I}}\right\} .
\end{aligned}
$$

Then, $\mathcal{H}$ satisfies $\mathcal{A}$ as shown below:

- For each concept assertion $D(b)$ in $\mathcal{A}$, as $b^{\mathcal{I}} \in D^{\mathcal{I}}$, by Lemma 3.4 $\tau^{\mathcal{I}}\left(b^{\mathcal{I}}\right)$ satisfies $D$, and since $\tau^{\mathcal{H}}(b)=\tau^{\mathcal{I}}\left(b^{\mathcal{I}}\right), \tau^{\mathcal{H}}(b)$ satisfies $D$.

- For each role assertion $R(b, c)$ in $\mathcal{A}$, as $\left(b^{\mathcal{I}}, c^{\mathcal{I}}\right) \in R^{\mathcal{I}}, R(b, c)$ is in $\mathcal{H}$.

- For each role assertion $\neg R(b, c)$ in $\mathcal{A}$, as $\left(b^{\mathcal{I}}, c^{\mathcal{I}}\right) \notin R^{\mathcal{I}}, R(b, c)$ is not in $\mathcal{H}$.

However, since $a^{\mathcal{I}} \notin C^{\mathcal{I}}$, by Lemma 3.4 $\tau^{\mathcal{H}}(a)$ does not satisfy $C$. That is, $\mathcal{H}$ does not satisfy $C(a)$, and thus, $\mathcal{A} \not \nvdash_{h} C(a)$.

For the "only if" direction, suppose that $\mathcal{A} \nvdash_{h} C(a)$, i.e., there is a Herbrand set $\mathcal{H}$ satisfying $\mathcal{A}$ but not $C(a)$. We show that $\mathcal{H}$ can be extended into an interpretation $\mathcal{I}$. Note that $\mathcal{H}$ is almost a DL interpretation, except that for each concept assertion of the form $\geqslant n P(a)$ in $\mathcal{H}, \mathcal{H}$ does not necessarily contain $n$ different role assertions $P\left(a, b_{i}\right)$, which is needed for a DL interpretation but not required by the definition of a Herbrand set. Hence, we need to extend $\mathcal{H}$ with such missing role assertions. Recall that for each type $\tau$ and each role $R \in \mathcal{S}_{R} \cup \mathcal{S}_{R}^{-}$, suc $R(\tau)$ is defined as in the proof of Proposition 3.5. We extend $\mathcal{H}$ into $\mathcal{H}^{*}$ as follows: for each individual $b \in \mathcal{S}_{I}$ and each role $R \in \mathcal{S}_{R} \cup \mathcal{S}_{R}^{-}$, if $R\left(b, c_{i}\right)(i=1, \ldots, n)$ are all the role assertions of such form in $\mathcal{H}$ and $\operatorname{suc}_{R}\left(\tau^{\mathcal{H}}(b)\right)>n$, then add $\operatorname{suc}_{R}\left(\tau^{\mathcal{H}}(b)\right)-n$ role assertions of the form $R(b, c)$ to $\mathcal{H}$ where each $c$ is a fresh individual.

We then construct an interpretation $\mathcal{I}$ form $\mathcal{H}^{*}$ as follows:

— Let $\Delta^{\mathcal{I}}$ be the set of all individuals in $\mathcal{H}$ and the newly introduced individuals.

- For each $b \in \mathcal{S}_{I}$, let $b^{\mathcal{I}}=b$.

- For each $A \in \mathcal{S}_{C}$, let $A^{\mathcal{I}}=\left\{b \mid A(b) \in \mathcal{H}^{*}\right\}$.

— For each $P \in \mathcal{S}_{R}$, let $P^{\mathcal{I}}=\left\{(b, c) \mid P(b, c) \in \mathcal{H}^{*}\right\}$.

From the construction of $\mathcal{I}$, we can see that for each individual $b \in \mathcal{S}_{I}$, the type induced by $b^{\mathcal{I}}$ in $\mathcal{I}$, i.e., $\tau^{\mathcal{I}}\left(b^{\mathcal{I}}\right)$, is exactly the type of $b$ in $\mathcal{H}$, i.e., $\tau^{\mathcal{H}}(b)$.

Then, $\mathcal{I}$ satisfies $\mathcal{A}$ as shown below:

- For each concept assertion $D(b)$ in $\mathcal{A}$, as $\tau^{\mathcal{H}}(b)$ satisfies $D$, and since $\tau^{\mathcal{H}}(b)=\tau^{\mathcal{I}}\left(b^{\mathcal{I}}\right)$, by Lemma 3.4. $b^{\mathcal{I}} \in D^{\mathcal{I}}$.

- For each role assertion $R(b, c)$ in $\mathcal{A}$, as $R(b, c)$ is in $\mathcal{H},\left(b^{\mathcal{I}}, c^{\mathcal{I}}\right) \in R^{\mathcal{I}}$.

— For each role assertion $\neg R(b, c)$ in $\mathcal{A}$, as $R(b, c)$ is not in $\mathcal{H},\left(b^{\mathcal{I}}, c^{\mathcal{I}}\right) \notin R^{\mathcal{I}}$.

Also, by Lemma 3.4, we have $\mathcal{I}$ does not satisfy $C(a)$.

Proposition 3.13 Given a featured interpretation $\mathcal{F}$, there always exists an interpretation $\mathcal{I}$ such that $\mathcal{F}_{\mathcal{I}}=\mathcal{F}$. 
Proof Suppose $\mathcal{F}=\langle\Xi, \mathcal{H}\rangle$, we construct an interpretation $\mathcal{I}$ from $\mathcal{F}$ in a similar way as in the proof of Proposition 3.5. The difference here is that we are going to construct a forest $F$ (i.e., a set of trees whose roots are arbitrarily connected) that induces the interpretation $\mathcal{I}$, which is a forest-shaped interpretation.

Given $\tau \in \Xi$ and $R \in \mathcal{S}_{R} \cup \mathcal{S}_{R}^{-}, \operatorname{suc}_{R}(\tau)$ is defined in the same way as before. For a Herbrand set $\mathcal{H}$ and an individual $a$ in $\mathcal{H}$, let $\operatorname{suc}_{R}(a, \mathcal{H})=|\{b \mid R(a, b) \in \mathcal{H}\}|$ be the number of R-successors of $a$ in $\mathcal{H}$. $F$ can be divided into two parts, the root part and tree-shaped part. The root part of $F$ consists of all the root nodes which can be interconnected arbitrarily, and in particular, in a non-tree way. The root nodes in $F$ and the edges between them are exactly as follows:

— there is one root node $d_{\tau}$, labelled with $\tau$ for each $\tau \in \Xi$;

- there is one root node $d_{a}$, labelled with $\tau^{\mathcal{H}}(a)$, for each individual $a$ in $\mathcal{H}$;

- there is an edge labelled with $P$ from root node $d_{a}$ to another root node $d_{b}$ iff $P(a, b) \in \mathcal{H}$.

The tree-shaped part of $F$ is constructed in the same way as in the proof of Proposition 3.5. For each node $d$ labelled with $\tau$ and each role $R \in \mathcal{S}_{R} \cup \mathcal{S}_{R}^{-}$, the following two conditions must be satisfied: (1) $d$ has $n R$-children $d_{i}(i=1, \ldots, n)$ in the tree-shaped part, where $n=s u c_{R}(\tau)-k$ with $k$ being the number of all $R$-successors and $R^{-}$-predecessors of $d$ already existing in the root part or on the path to $d$ in $F$. In particular,

- if $d$ is a root node $d_{a}$ and $\operatorname{suc}_{R}(\tau)>\operatorname{suc}_{R}(a, \mathcal{H})$, then $k=\operatorname{suc}_{R}(a, \mathcal{H})$;

- if $d$ is a non-root node and an $R^{-}$-child, then $k=1$; and

- otherwise, $k=0$.

(2) Each $R$-child $d_{i}$ is labelled with a type $\tau_{i}$ such that $s u c_{R^{-}}\left(\tau_{i}\right)>0$.

To show that we can construct a forest $F$ satisfying the above conditions, note that meeting the conditions for the root part is straightforward. For the tree-shaped part, Condition (2) requires a type $\tau_{i}$ containing $\exists R^{-}$in $\Xi$ to label each $R$-child. Note that if $d$ has an $R$-child then $\operatorname{suc}_{R}(\tau)>0$, that is $\tau$ contains $\exists R$. From the definition of a featured interpretation, there exists a $\tau_{R^{-}} \in \Xi$ containing $\exists R^{-}$, i.e., $s u c_{R^{-}}\left(\tau_{R^{-}}\right)>0$. Hence, we can take $\tau_{i}=\tau_{R^{-}}$from $\Xi$. For Condition (1) to be satisfied, we only need to make sure $n \geq 0$. It is clear if $d$ is not an $R^{-}$-child; when $d$ is an $R^{-}$-child, by Condition (2), we have $\operatorname{suc}_{R}(\tau)>0$ and thus $n=\operatorname{suc}_{R}(\tau)-1 \geq 0$.

We define interpretation $\mathcal{I}$ from $F$ in an obvious way:

- Let $\Delta^{\mathcal{I}}$ be the set of the nodes in $F$.

- For each $a \in \mathcal{S}_{I}$, let $a^{\mathcal{I}}=d_{a}$.

- For each $A \in \mathcal{S}_{C}$, let $A^{\mathcal{I}}=\{d \mid d$ has label $\tau$ containing $A\}$.

- For each $P \in \mathcal{S}_{R}$, let $P^{\mathcal{I}}=\left\{(d, e) \mid(d, e)\right.$ has label $P$ or $(e, d)$ has label $\left.P^{-}\right\}$.

From the construction of $\mathcal{I}$, we can see that $\mathcal{I}$ induces $\mathcal{F}$.

Proposition 3.24 For a set $\mathbb{F}$ of featured interpretations, $\mathbb{F}$ is axiomatizable in DL-Lite $\mathcal{L}_{\text {bool }}^{\mathcal{N}}$ iff $\mathbb{F}$ is closed under $\bigoplus$.

Proof $\quad$ Suppose $\mathbb{F}=\left\{\mathcal{F}_{1}, \ldots, \mathcal{F}_{n}\right\}$ with $\mathcal{F}_{i}=\left\langle\Xi_{i}, \mathcal{H}_{i}\right\rangle(1 \leq i \leq n)$.

The "only if" direction: Suppose $\mathbb{F}$ is axiomatizable in DL-Lite $\mathrm{b}_{\text {bool }}^{\mathcal{N}}$, then there exists a DL-Lite $\mathcal{L}_{\text {bool }}^{\mathcal{N}}$ $\mathrm{KB} \mathcal{K}$ such that $\mathrm{FM}(\mathcal{K})=\mathbb{F}$. For each featured interpretation $\mathcal{F}=\langle\Xi, \mathcal{H}\rangle$ satisfying Conditions (1)-(3) in the definition of $\bigoplus$, we want to show that $\mathcal{F} \in \mathbb{F}$. We only need to show that $\mathcal{F} \in \mathrm{FM}(\mathcal{K})$, that is, $\mathcal{F}$ satisfies $\mathcal{K}$.

— $\Xi$ satisfies $\mathcal{T}$ as each type in $\bigcup_{1 \leq i \leq n} \Xi_{i}$ satisfies $\mathcal{T}$.

- For each concept assertion $C(a)$ in $\mathcal{A}$, as $C A(a, \mathcal{H})=C A\left(a, \mathcal{H}_{i}\right)$ for some $i$ and $\tau^{\mathcal{H}_{i}}(a)$ satisfies $C$, we have $\mathcal{H}$ satisfies $C(a)$.

— For each role assertion $P(a, b)$ in $\mathcal{A}, P(a, b)$ must be in each $\mathcal{H}_{i}$, that is, $P(a, b) \in R A(\mathcal{H})$. For each role assertion $\neg P(a, b)$ in $\mathcal{A}, P(a, b)$ is not in any $\mathcal{H}_{i}$, and similarly, $P(a, b) \notin R A(\mathcal{H})$. We have shown that $\mathcal{H}$ satisfies each role assertion in $\mathcal{A}$. 
We have shown that $\mathcal{F}$ is in $\operatorname{FM}(\mathcal{K})$.

The "if" direction: We first construct a $\mathrm{KB} \mathcal{K}$ from $\mathbb{F}$, and then show that $\mathrm{FM}(\mathcal{K})$ is exactly $\mathbb{F}$. For a type $\tau$, let $\operatorname{Con}(\tau)=\prod_{B \in \tau} B \sqcap \prod_{B \in B C_{\mathcal{S}} \backslash \tau} \neg B$. It is clear that $\tau$ satisfies $C o n(\tau)$ and $\tau$ does not satisfy $\operatorname{Con}\left(\tau^{\prime}\right)$ for any type $\tau^{\prime}$ with $\tau^{\prime} \neq \tau$. Then we can construct $\mathcal{K}=\left\langle\mathcal{T}, \mathcal{A}_{c} \cup \mathcal{A}_{r}\right\rangle$ where

$$
\begin{aligned}
\mathcal{T} & =\left\{\top \sqsubseteq \bigsqcup_{1 \leq i \leq n} \bigsqcup_{\tau \in \Xi_{i}} \operatorname{Con}(\tau)\right\} . \\
\mathcal{A}_{c} & =\left\{\left(\bigsqcup_{1 \leq i \leq n} \operatorname{Con}\left(\tau_{i}\right)\right)(a) \mid a \in \mathcal{S}_{I}, \tau_{i}=\tau^{\mathcal{H}_{i}}(a)\right\} . \\
\mathcal{A}_{r} & =\left(\bigcap_{1 \leq i \leq n} R A\left(\mathcal{H}_{i}\right)\right) \cup\left\{\neg P(a, b) \mid P \in \mathcal{S}_{R}, a, b \in \mathcal{S}_{I}, P(a, b) \notin \bigcup_{1 \leq i \leq n} R A\left(\mathcal{H}_{i}\right)\right\} .
\end{aligned}
$$

For each $\mathcal{F}_{i}$, similar as above, it is not hard to verify that $\mathcal{F}_{i}$ satisfies $\mathcal{K}$. That is, $\mathbb{F} \subseteq \operatorname{FM}(\mathcal{K})$.

Conversely, for each featured model $\mathcal{F}=\langle\Xi, \mathcal{H}\rangle$ of $\mathcal{K}$, we show that $\mathcal{F}$ is in $\mathbb{F}$ as follows. We achieve this by showing $\mathcal{F} \in \bigoplus \mathbb{F}$.

- $\Xi$ satisfying $\mathcal{T}$ implies that each $\tau \in \Xi$ must satisfy some concept $\operatorname{Con}(\tau)$ in $\bigsqcup_{1 \leq i \leq n} \bigsqcup_{\tau \in \Xi_{i}} \operatorname{Con}(\tau)$. That is, $\mathcal{F}$ satisfies Condition (1) in the definition of the $\bigoplus \mathbb{F}$ closure.

$-\mathcal{H}$ satisfying $C(a)$ in $\mathcal{A}_{c}$ implies that $\tau^{\mathcal{H}}(a)$ must satisfy $\operatorname{Con}(\tau)$ for some $\tau \in \Xi_{a}$. That is, $\tau^{\mathcal{H}}(a)=\tau^{\mathcal{H}_{i}}(a)$ for some $1 \leq i \leq n$ and $\mathcal{F}$ satisfies Condition (2) of $\bigoplus \mathbb{F}$.

$-\mathcal{H}$ satisfying $\mathcal{A}_{r}$ implies $\bigcap_{1 \leq i \leq n} R A\left(\mathcal{H}_{i}\right) \subseteq R A(\mathcal{H}) \subseteq \bigcup_{1 \leq i \leq n} R A\left(\mathcal{H}_{i}\right)$. That is, $\mathcal{F}$ satisfies Condition (3) of $\bigoplus \mathbb{F}$.

That is, $\mathcal{F} \in \bigoplus \mathbb{F}$. Since $\mathbb{F}$ is closed under $\bigoplus, \mathcal{F}$ is in $\mathbb{F}$. Thus, we have shown $\operatorname{FM}(\mathcal{K})=\mathbb{F}$.

Lemma 3.25 For a $K B \mathcal{K}$ over $\mathcal{S}$ and a $K B \mathcal{K}^{\prime}$ over $\mathcal{S}^{\prime}$ such that $\mathcal{K}^{\prime} \backslash \mathcal{K}=\left\{C_{U} \sqsubseteq \exists U, \exists U^{-} \sqsubseteq\right.$ $\left.D_{U} \mid U \in \mathcal{S}^{\prime} \backslash \mathcal{S}\right\}$ where each $C_{U}$ and each $D_{U}$ are over $\mathcal{S}$, an $\mathcal{S}$-featured interpretation $\mathcal{F}$ is an $\mathcal{S}$-featured model of $\mathcal{K}^{\prime}$ iff (1) $\mathcal{F}$ is an featured model of $\mathcal{K}$ and (2) $\mathcal{F}$ satisfies concept $D_{U}$ whenever it satisfies $C_{U}$ for each $U \in \mathcal{S}^{\prime} \backslash \mathcal{S}$.

Proof We first show the following observation: for an $\mathcal{S}$-featured model $\mathcal{F}$, an $\mathcal{S}^{\prime}$-extension $\mathcal{F}^{\prime}$ of $\mathcal{F}$, a concept $C$ over $\mathcal{S}$, and an axiom $\alpha$ over $\mathcal{S}, \mathcal{F}$ satisfies $C$ iff $\mathcal{F}^{\prime}$ satisfies $C$, and $\mathcal{F}$ satisfies $\alpha$ iff $\mathcal{F}^{\prime}$ satisfies $\alpha$. From the definition of a type satisfying a concept, a type $\tau$ in $\mathcal{F}$ satisfies $C$ iff an (arbitrary) $\mathcal{S}^{\prime}$-extension of $\tau$ satisfies $C$. Hence, $\mathcal{F}$ satisfies $C$ iff $\mathcal{F}^{\prime}$ satisfies $C$. Suppose $\alpha$ is a concept inclusion $C \sqsubseteq D$, for each type $\tau$ in $\mathcal{F}, \tau$ satisfies $C \sqsubseteq D$ iff $\tau$ satisfies concept $\neg C \sqcup D$ iff an (arbitrary) $\mathcal{S}^{\prime}$-extension $\tau^{\prime}$ of $\tau$ satisfies concept $\neg C \sqcup \bar{D}$, iff $\tau^{\prime}$ satisfies $C \sqsubseteq D$. Hence, $\mathcal{F}$ satisfies $C \sqsubseteq D$ iff $\mathcal{F}^{\prime}$ satisfies $C \sqsubseteq D$. Similarly, suppose $\alpha$ is an assertion, $\mathcal{F}$ satisfies $\alpha$ iff its Herbrand set $\mathcal{H}$ satisfies $\alpha$, iff the Herbrand set of $\mathcal{F}^{\prime}$ (that is an $\mathcal{S}^{\prime}$-extension of $\mathcal{H}$ ) satisfies $\alpha$, iff $\mathcal{F}^{\prime}$ satisfies $\alpha$.

To prove the "if" direction of the the lemma, we only need to show that an $\mathcal{S}^{\prime}$-extension of $\mathcal{F}$ exists that satisfies $\mathcal{K}^{\prime}$. Suppose $\mathcal{F}=\langle\Xi, \mathcal{H}\rangle$. Let $\mathcal{F}^{\prime}$ be obtained from $\mathcal{F}$ by the following steps:

(i) For each $U \in \mathcal{S}^{\prime} \backslash \mathcal{S}$ and each type $\tau$ in $\Xi$, if $\tau$ satisfies $C_{U}$ then add $\exists U$ to $\tau$.

(ii) If step (i) is triggered then $\mathcal{F}$ satisfies $C_{U}$. By (2), $\mathcal{F}$ satisfies $D_{U}$ and there must be a type $\tau^{\prime}$ in $\Xi$ satisfying $D_{U}$. Add $\exists U^{-}$to $\tau^{\prime}$.

(iii) For each $a \in \mathcal{S}_{I}$ and the type $\tau=\tau^{\mathcal{H}}(a)$ induced by $a$, note that $\tau \in \Xi$ by Definition 3.10 If $\exists U$ (or $\exists U^{-}$) is added to $\tau$ in step (i) (resp. step (ii)), then add $\exists U(a)$ (resp., $\exists U^{-}(a)$ ) to $\mathcal{H}$.

We show that $\mathcal{F}^{\prime}$ is an $\mathcal{S}^{\prime}$-featured interpretation. For Condition (1) of Definition 3.10, if a type in $\mathcal{F}^{\prime}$ contains some $\exists U$ then step (i) was triggered, which in turn triggers step (ii). Hence, $\exists U^{-}$must be contained by some type in $\mathcal{F}^{\prime}$. Also, it is clear that Condition (2) of Definition 3.10 is ensured by step (iii). Hence, $\mathcal{F}^{\prime}$ is an $\mathcal{S}^{\prime}$-extension of $\mathcal{F}$. We only need to show that $\mathcal{F}^{\prime}$ satisfies $\mathcal{K}^{\prime}$. From the above observation, as $\mathcal{F}$ satisfies $\mathcal{K}, \mathcal{F}^{\prime}$ also satisfies $\mathcal{K}$. Also, for each $U \in \mathcal{S}^{\prime} \backslash \mathcal{S}, \mathcal{F}^{\prime}$ satisfies $C_{U} \sqsubseteq \exists U$, since each type in $\mathcal{F}^{\prime}$ satisfying $C_{U}$ also contains $\exists U$. Further, $\mathcal{F}^{\prime}$ satisfies $\exists U^{-} \sqsubseteq D_{U}$, 
since for each type $\tau^{\prime}$ in $\mathcal{F}^{\prime}$ containing $\exists U^{-}, \tau^{\prime}$ also contains $D_{U}$. We have shown that $\mathcal{F}^{\prime}$ satisfies $\mathcal{K}^{\prime}$ and thus is a featured model of $\mathcal{K}^{\prime}$.

To prove the "only if" direction, we note that $\mathcal{F}$ being an $\mathcal{S}$-featured model of $\mathcal{K}^{\prime}$ implies that an $\mathcal{S}^{\prime}$-extension $\mathcal{F}^{\prime}$ of $\mathcal{F}$ satisfies $\mathcal{K}^{\prime}$. That is, $\mathcal{F}^{\prime}$ satisfies $\mathcal{K}$, and hence, from the above observation, $\mathcal{F}$ satisfies $\mathcal{K}$. We have shown that $\mathcal{F}$ is a featured model of $\mathcal{K}$. Moreover, $\mathcal{F}^{\prime}$ satisfies each $C_{U} \sqsubseteq \exists U$ and each $\exists U^{-} \sqsubseteq D_{U}$ in $\mathcal{K}^{\prime}$. For each $U \in \mathcal{S}^{\prime} \backslash \mathcal{S}$, if $\mathcal{F}$ satisfies $C_{U}$, then by the above observation, $\mathcal{F}^{\prime}$ satisfies $C_{U}$. Since $\mathcal{F}^{\prime}$ satisfies $C_{U} \sqsubseteq \exists U, \mathcal{F}^{\prime}$ satisfies $\exists U$, that is, a type in $\mathcal{F}^{\prime}$ contains $\exists U$. By Condition (1) of Definition 3.10, there exists a type $\tau^{\prime}$ in $\mathcal{F}^{\prime}$ containing $\exists U^{-}$. Since $\mathcal{F}^{\prime}$ satisfies $\exists U^{-} \sqsubseteq D_{U}, \tau^{\prime}$ must satisfy $D_{U}$. By the definition of $\mathcal{S}^{\prime}$-extension, $\tau^{\prime}$ is an $\mathcal{S}^{\prime}$-extension of some type $\tau$ in $\mathcal{F}$. Then, by the above observation, $\tau$ satisfies $D_{U}$. That is, $\mathcal{F}$ satisfies $D_{U}$. We have shown that $\mathcal{F}$ satisfies $D_{U}$ whenever it satisfies $C_{U}$ for each $U \in \mathcal{S}^{\prime} \backslash \mathcal{S}$.

Proposition 3.26 A set $\mathbb{F}$ of featured interpretations is always DKB-axiomatizable in DL-Lite $e_{\text {bool }}^{\mathcal{N}}$.

Proof We show that for each $\mathcal{S}$-featured interpretation $\mathcal{F}=\langle\Xi, \mathcal{H}\rangle$, there exists a DL-Lite $\mathcal{E}_{\text {bool }}^{\mathcal{N}}$ $\mathrm{KB} \mathcal{K}$ over an extended signature $\mathcal{S}^{\prime}=\mathcal{S} \cup\left\{U_{\tau} \mid \tau \in \Xi\right\}$, where each $U_{\tau}$ is a fresh role name, such that $\operatorname{FM}(\mathcal{K})=\{\mathcal{F}\}$. Such a $\mathrm{KB} \mathcal{K}=\left\langle\mathcal{T}, \mathcal{A}_{c} \cup \mathcal{A}_{r}\right\rangle$ can be constructed as follows:

$$
\begin{aligned}
\mathcal{T} & =\left\{\top \sqsubseteq \bigsqcup_{\tau \in \Xi} \operatorname{Con}(\tau)\right\} \cup\left\{\top \sqsubseteq \exists U_{\tau}, \exists U_{\tau}^{-} \sqsubseteq \operatorname{Con}(\tau) \mid \tau \in \Xi\right\} \\
\mathcal{A}_{c} & =\bigcup_{a \in \mathcal{S}_{I}} C A(a, \mathcal{H}) \\
\mathcal{A}_{r} & =R A(\mathcal{H}) \cup\left\{\neg P(a, b) \mid P \in \mathcal{S}_{R}, a, b \in \mathcal{S}_{I}, P(a, b) \notin R A(\mathcal{H})\right\} .
\end{aligned}
$$

where $\operatorname{Con}(\tau)$ is defined as in the proof of Proposition 3.24 .

Similar as the proof of Proposition 3.24 one can verify that $\mathcal{F}$ satisfies all the axioms in $\mathcal{K}$ except for those of the forms $T \sqsubseteq \exists U_{\tau}$ and $\exists U_{\tau}^{-} \sqsubseteq \operatorname{Con}(\tau)$. Note that $\mathcal{F}$ satisfies each concept $\operatorname{Con}(\tau)$ as type $\tau$ in $\mathcal{F}$ satisfies $\operatorname{Con}(\tau)$. From Lemma 3.25, $\mathcal{F}$ is a featured model of $\mathcal{K}$. Conversely, for each $\mathcal{S}$-featured model $\mathcal{F}^{\prime}=\left\langle\Xi^{\prime}, \mathcal{H}^{\prime}\right\rangle$ of $\mathcal{K}$, we show that $\mathcal{F}^{\prime}=\mathcal{F}$. For each type $\tau^{\prime} \in \Xi^{\prime}$, since $\tau^{\prime}$ satisfies $T \sqsubseteq \bigsqcup_{\tau \in \Xi} \operatorname{Con}(\tau), \tau^{\prime}$ must satisfy some $\operatorname{Con}(\tau)$, which implies that $\tau^{\prime}=\tau$ for some $\tau \in \Xi$. That is, $\tau^{\prime} \in \Xi$ and hence $\Xi^{\prime} \subseteq \Xi$. Also, for each type $\tau \in \Xi$, since $\Xi^{\prime}$ satisfies $\top \sqsubseteq \exists U_{\tau}$ and $\exists U_{\tau}^{-} \sqsubseteq \operatorname{Con}(\tau)$, there must be a type in $\Xi^{\prime}$ that satisfies $\operatorname{Con}(\tau)$. That is, $\tau \in \Xi^{\prime}$ and hence $\Xi \subseteq \Xi^{\prime}$. We have $\Xi=\Xi^{\prime}$. Similar as the proof of Proposition 3.24, we can show that $\mathcal{H}=\mathcal{H}^{\prime}$. Hence, $\mathcal{F}^{\prime}=\mathcal{F}^{\prime}$, and we have shown $\operatorname{FM}(\mathcal{K})=\{\mathcal{F}\}$.

Suppose $\mathbb{F}=\left\{\mathcal{F}_{1}, \ldots, \mathcal{F}_{n}\right\}$, then there is a DKB $\mathbb{K}=\left\{\mathcal{K}_{1}, \ldots, \mathcal{K}_{n}\right\}$ such that $\operatorname{FM}\left(\mathcal{K}_{i}\right)=\left\{\mathcal{F}_{i}\right\}$ for $1 \leq i \leq n$. That is, $\operatorname{FM}(\mathbb{K})=\mathbb{F}$.

Lemma 3.28 Given a set $\mathbb{M}$ of interpretations and a signature $\mathcal{S}$, let $\mathbb{F}=\left\{\mathcal{F}_{\mathcal{I}} \mid \mathcal{I} \in \mathbb{M}\right\}$. The MA of $\mathbb{F}$ in DL-Lite $e_{\text {bool }}^{\mathcal{N}}$ is exactly the MA of $\mathbb{M}$ over $\mathcal{S}$ in DL-Lite $e_{\text {bool. }}^{\mathcal{N}}$.

Proof Suppose DL-Lite ${ }_{\text {bool }}^{\mathcal{N}} \mathrm{KB} \mathcal{K}$ is the MA of $\mathbb{F}$, then $\operatorname{sig}(\mathcal{K}) \subseteq \mathcal{S}$. We want to show that $\mathcal{K}$ is the $\mathrm{MA}$ of $\mathbb{M}$. For each interpretation $\mathcal{I} \in \mathbb{M}$, since $\mathbb{F} \subseteq \mathrm{FM}(\mathcal{K}), \mathcal{F}_{\mathcal{I}}$ satisfies $\mathcal{K}$. By Corollary 3.16 , $\mathcal{I}$ is a model of $\mathcal{K}$. That is, $\mathbb{M} \subseteq \bmod (\mathcal{K})$. Moreover, for each DL-Lite bool $^{\mathcal{N}}$ KB $\mathcal{K}^{\prime}$ over $\mathcal{S}$ such that $\mathbb{M} \subseteq \bmod \left(\mathcal{K}^{\prime}\right)$, from Corollary 3.16, each featured interpretation in $\mathbb{F}$ satisfies $\mathcal{K}^{\prime}$. That is, $\mathbb{F} \subseteq \mathrm{FM}\left(\mathcal{K}^{\prime}\right)$ and thus $\mathbb{F} \subseteq \mathrm{FM}(\mathcal{K}) \cap \mathrm{FM}\left(\mathcal{K}^{\prime}\right)$. By Proposition 3.18, $\mathbb{F} \subseteq \mathrm{FM}\left(\mathcal{K} \cup \mathcal{K}^{\prime}\right)$. Since $\mathcal{K}$ is the MA of $\mathbb{F}, \operatorname{FM}(\mathcal{K})=\operatorname{FM}\left(\mathcal{K} \cup \mathcal{K}^{\prime}\right) \subseteq \operatorname{FM}\left(\mathcal{K}^{\prime}\right)$. From Theorem 3.20, $\mathcal{K} \models \mathcal{K}^{\prime}$. That is, $\bmod (\mathcal{K}) \subseteq \bmod \left(\mathcal{K}^{\prime}\right)$. We have shown that $\mathcal{K}$ is the MA of $\mathbb{M}$.

\section{B. PROOFS FOR SECTION 4}

Proposition 4.4 Let $\mathcal{K}, \mathcal{K}^{\prime}$ be two consistent DL-Lite bool $_{\mathcal{N}}^{\mathcal{N}} K$ s and $\operatorname{sig}\left(\mathcal{K} \cup \mathcal{K}^{\prime}\right) \subseteq \mathcal{S}$. Then,

$$
\mathcal{K} \circ_{P} \mathcal{K}^{\prime}=\left\{\text { forget }^{U}(\mathcal{K}, \Sigma) \cup \mathcal{K}^{\prime} \mid \Sigma \in \operatorname{MD}_{P}\left(\mathcal{K}, \mathcal{K}^{\prime}\right)\right\} .
$$

To prove Proposition 4.4, we use the following result from [Wang et al. 2010c].In particular, an equivalent characterisation of forgetting by featured models is given as follows. 
Lemma B.1. Let $\mathcal{K}$ be a $K B, \Sigma \subseteq \Sigma_{\mathcal{S}}$ be a set of predicates, and $\mathcal{S}^{\prime}=\mathcal{S} \backslash \Sigma$. For a $K B \mathcal{K}^{\prime}$ over $\mathcal{S}^{\prime}$, forget $(\mathcal{K}, \Sigma) \equiv \mathcal{K}^{\prime}$ iff (1) $\mathcal{K} \models \mathcal{K}^{\prime}$; and (2) for each model $\mathcal{I}^{\prime} \in \bmod \left(\mathcal{K}^{\prime}\right)$, there exists $\mathcal{I} \in \bmod (\mathcal{K})$ such that $\mathcal{I}$ and $\mathcal{I}^{\prime}$ induce the same $\mathcal{S}^{\prime}$-featured interpretation.

Also, we show the following lemma that states the featured interpretations induced by the DL models of forget $(\mathcal{K}, \Sigma)$ are exactly $\mathrm{FM}\left(\right.$ forget $\left.^{U}(\mathcal{K}, \Sigma)\right)$. Recall that DL-Lite bool $_{\text {extends DL-Lite }}^{\mathcal{N}}$ exol $_{\text {bol }}^{\mathcal{N}}$ by introducing new concepts of the form $\exists u$.C to TBoxes, where $C$ is a concept in DL-Lite $\mathrm{bool}^{\mathcal{N}}$. Given an interpretation $\mathcal{I},(\exists u . C)^{\mathcal{I}}=\Delta^{\mathcal{I}}$ if $C^{\mathcal{I}} \neq \emptyset$ and $(\exists u . C)^{\mathcal{I}}=\emptyset$ if $C^{\mathcal{I}}=\emptyset$.

LEMMA B.2. Let $\mathcal{K}$ be a $K B, \Sigma \subseteq \Sigma_{\mathcal{S}}$ be a set of predicates. Then, $\mathrm{FM}\left(\right.$ forget $\left.^{U}(\mathcal{K}, \Sigma)\right)=$ $\left\{\mathcal{F}_{I} \mid \mathcal{I} \in \bmod (\right.$ forget $\left.(\mathcal{K}, \Sigma))\right\}$, and $\operatorname{FM}\left(\right.$ forget $\left.{ }^{U}(\mathcal{K}, \Sigma)\right)=\left\{\mathcal{F}^{\prime} \mid \mathcal{F}^{\prime} \sim_{\bar{\Sigma}} \mathcal{F}\right.$ for some $\mathcal{F} \in$ $\operatorname{FM}(\mathcal{K})\}$.

Proof Let $\mathcal{K}^{\prime}=$ forget $(\mathcal{K}, \Sigma), \mathcal{K}^{\prime \prime}=$ forget $^{U}(\mathcal{K}, \Sigma)$, and $\mathbb{F}=\left\{\mathcal{F}^{\prime} \mid \mathcal{F}^{\prime} \sim_{\bar{\Sigma}} \mathcal{F}\right.$ for some $\mathcal{F} \in$ $\operatorname{FM}(\mathcal{K})\}$. To show the first half of the lemma, we want to show firstly that for each $\mathcal{I} \in \bmod \left(\mathcal{K}^{\prime}\right)$, $\mathcal{F}_{\mathcal{I}}$ is a featured model $\mathcal{K}^{\prime \prime}$. By Proposition 3.15, $\mathcal{F}_{\mathcal{I}}$ satisfies each axiom in $\mathcal{K}^{\prime}$ except for those of the form $C \sqsubseteq \exists u . D$. That is, $\mathcal{F}_{\mathcal{I}}$ satisfies each axiom in $\mathcal{K}^{\prime}$ except for $C \sqsubseteq \exists U_{C, D}$ and $\exists U_{C, D}^{-} \sqsubseteq D$. If $\mathcal{F}_{\mathcal{I}}$ satisfies $C$, by Proposition 3.15. $C^{\mathcal{I}} \neq \emptyset$. Since $\mathcal{I}$ is a model of $C \sqsubseteq \exists u . D,(\exists u . D)^{\mathcal{I}} \neq \emptyset$, and that is, by the semantics of DL-Lite ${ }_{\text {bool }}^{u}, D^{\mathcal{I}} \neq \emptyset$. Again, by Proposition 3.15 . $\mathcal{F}_{\mathcal{I}}$ satisfies $D$. That is, $\mathcal{F}_{\mathcal{I}}$ satisfies $D$ whenever it satisfies $C$. By Lemma 3.25, $\mathcal{F}_{\mathcal{I}}$ is a featured model of $\mathcal{K}^{\prime \prime}$.

Conversely, we want to show that for each $\mathcal{F} \in \mathrm{FM}\left(\mathcal{K}^{\prime \prime}\right)$, there is a DL model $\mathcal{I}$ of $\mathcal{K}^{\prime}$ that induces $\mathcal{F}$. By Proposition 3.13, there is a DL model $\mathcal{I}$ of $\mathcal{K}^{\prime \prime}$ that induces $\mathcal{F}$. That is, $\mathcal{I}$ satisfies every axiom in $\mathcal{K}^{\prime \prime}$, and hence satisfies each axiom in $\mathcal{K}^{\prime}$ except for those of the form $C \sqsubseteq \exists u . D$. If $C^{\mathcal{I}}=\emptyset$, then $\mathcal{I}$ satisfies $C \sqsubseteq \exists u$.D. Otherwise, if $C^{\mathcal{I}} \neq \emptyset$, note that $\mathcal{I}$ satisfies $C \sqsubseteq \exists \bar{U}_{C, D}$ and $\exists U_{C, D}^{-} \sqsubseteq D$. Hence, $U_{C, D}^{\mathcal{I}} \neq \emptyset$ and $D^{\mathcal{I}} \neq \emptyset$. by the semantics of DL-Lite bool,$(\exists u . D)^{\mathcal{I}}=\Delta^{\mathcal{I}}$. Again, $\mathcal{I}$ satisfies $C \sqsubseteq \exists u . D$. We have shown that $\mathcal{I}$ is a DL model of $\mathcal{K}^{\prime}$.

To show the second half of the lemma, we want to show that $\mathbb{F}=\left\{\mathcal{F}_{I} \mid \mathcal{I} \in \bmod \left(\mathcal{K}^{\prime}\right)\right\}$. We first show that $\left\{\mathcal{F}_{I} \mid \mathcal{I} \in \bmod \left(\mathcal{K}^{\prime}\right)\right\} \subseteq \mathbb{F}$. Let $\mathcal{S}^{\prime}=\mathcal{S} \backslash \Sigma$. For each $\mathcal{I} \in \bmod \left(\mathcal{K}^{\prime}\right)$, by Lemma B.1 (2), there exists a model $\mathcal{I}^{\prime}$ of $\mathcal{K}$ such that $\mathcal{I}$ and $\mathcal{I}^{\prime}$ induce the same $\mathcal{S}^{\prime}$-featured interpretation. Suppose $\mathcal{I}^{\prime}$ induces $\mathcal{S}$-featured interpretation $\mathcal{F}$, by Proposition 3.17, $\mathcal{F} \in \mathrm{FM}(\mathcal{K})$. We want to show that $\mathcal{F}_{\mathcal{I}} \sim_{\bar{\Sigma}} \mathcal{F}^{\prime}$. Suppose $\mathcal{F}_{\mathcal{I}}=\langle\Xi, \mathcal{H}\rangle$ and $\mathcal{F}^{\prime}=\left\langle\Xi^{\prime}, \mathcal{H}^{\prime}\right\rangle$. For an $\mathcal{S}$-type $\tau$, we denote $\left.\tau\right|_{\mathcal{S}^{\prime}}$ the projection of $\tau$ on $\mathcal{S}^{\prime}$, i.e., $\left.\tau\right|_{\mathcal{S}^{\prime}}$ is the maximal subset of $\tau$ using only symbols in $\mathcal{S}^{\prime}$. For each $\tau \in \Xi$, suppose $\tau$ is induced by $d$ in $\mathcal{I}$. Then, $\left.\tau\right|_{\mathcal{S}^{\prime}}$ is induced by $d$ in $\mathcal{I}$. Since, $\mathcal{I}$ and $\mathcal{I}^{\prime}$ induce the same $\mathcal{S}^{\prime}$-featured interpretation, there exists some $d^{\prime}$ in $\Delta^{\mathcal{I}^{\prime}}$ such that $d^{\prime}$ induces the same type $\left.\tau\right|_{\mathcal{S}^{\prime}}$ in $\mathcal{I}^{\prime}$. Let $\tau^{\prime}$ be the $\mathcal{S}$-type induced by $d^{\prime}$ in $\mathcal{I}^{\prime}$. Then, $\tau \sim_{\bar{\Sigma}} \tau^{\prime}$. Similarly, for each $\tau^{\prime} \in \Xi^{\prime}$, there is a type $\tau \in \Xi$ such that $\tau \sim_{\bar{\Sigma}} \tau^{\prime}$. For each $a \in \mathcal{S}_{I}, a^{\mathcal{I}}$ induces the same $\mathcal{S}^{\prime}$-type in $\mathcal{I}$ as $a^{\mathcal{I}^{\prime}}$ in $\mathcal{I}^{\prime}$. That is, $\tau^{\mathcal{H}}(a) \sim_{\bar{\Sigma}} \tau^{\mathcal{H}^{\prime}}(a)$. Moreover, $P(a, b) \in \mathcal{H}$ iff $P(a, b) \in \mathcal{H}^{\prime}$ for each $P \in \mathcal{S}^{\prime}$ and $a, b \in \mathcal{S}_{I}$. We have shown that $\mathcal{F}_{\mathcal{I}} \sim_{\bar{\Sigma}} \mathcal{F}^{\prime}$. That is, $\mathcal{F}_{\mathcal{I}} \in \mathbb{F}$. Thus, $\left\{\mathcal{F}_{I} \mid \mathcal{I} \in \bmod \left(\mathcal{K}^{\prime}\right)\right\} \subseteq \mathbb{F}$.

Conversely, we show that $\mathbb{F} \subseteq\left\{\mathcal{F}_{I} \mid \mathcal{I} \in \bmod \left(\mathcal{K}^{\prime}\right)\right\}$. For each $\mathcal{F} \in \mathbb{F}$, there exists a featured model $\mathcal{F}^{\prime} \in \operatorname{FM}(\mathcal{K})$ such that $\mathcal{F} \sim_{\bar{\Sigma}} \mathcal{F}^{\prime}$. From Lemma B.1 $(1), \bmod (\mathcal{K}) \subseteq \bmod \left(\mathcal{K}^{\prime}\right)$, and by Proposition 3.17 $\operatorname{FM}(\mathcal{K}) \subseteq\left\{\mathcal{F}_{I} \mid \mathcal{I} \in \bmod \left(\mathcal{K}^{\prime}\right)\right\}$. Thus, $\mathcal{F}^{\prime} \in\left\{\mathcal{F}_{I} \mid \mathcal{I} \in \bmod \left(\mathcal{K}^{\prime}\right)\right\}$. That is, there exists a DL model $\mathcal{I}^{\prime}$ of $\mathcal{K}^{\prime}$ that induces $\mathcal{F}^{\prime}$. By Proposition 3.13, there is a DL interpretation $\mathcal{I}$ that induces $\mathcal{F}$, and we want to show that $\mathcal{I}$ is a model of $\mathcal{K}^{\prime}$ too. For each axiom $\alpha$ in $\mathcal{K}^{\prime}$ other than those of the form $C \sqsubseteq \exists u . D, \mathcal{I}^{\prime}$ satisfies $\alpha$ and by Proposition 3.15, $\mathcal{F}^{\prime}$ satisfies $\alpha$. Since $\mathcal{F} \sim_{\bar{\Sigma}} \mathcal{F}^{\prime}$ and $\mathcal{K}^{\prime}$ does not contain any predicate in $\Sigma, \mathcal{F}$ also satisfies $\alpha$, and thus $\mathcal{I}$ also satisfies $\alpha$. For each axiom in $\mathcal{K}^{\prime}$ of the form $C \sqsubseteq \exists u . D, C^{\mathcal{I}^{\prime}} \neq \emptyset$ implies that $(\exists u . D)^{\mathcal{I}^{\prime}} \neq \emptyset$, which implies that $D^{\mathcal{I}^{\prime}} \neq \emptyset$. That is, by Proposition 3.15, $\mathcal{F}^{\prime}$ satisfying $C$ implies that $\mathcal{F}^{\prime}$ satisfying $D$. Similarly, as $C$ and $D$ are over $\bar{\Sigma}, \mathcal{F}$ satisfying $C$ implies that $\mathcal{F}$ satisfying $D$. That is, if $C^{\mathcal{I}} \neq \emptyset$ then $D^{\mathcal{I}} \neq \emptyset$, hence by the semantics of DL-Lite bool,$C^{\mathcal{I}} \subseteq(\exists u . D)^{\mathcal{I}}$. That is, $\mathcal{I} \in \bmod \left(\mathcal{K}^{\prime}\right)$ and $\mathcal{F} \in\left\{\mathcal{F}_{I} \mid \mathcal{I} \in \bmod \left(\mathcal{K}^{\prime}\right)\right\}$. We have shown that $\mathbb{F} \subseteq\left\{\mathcal{F}_{I} \mid \overline{\mathcal{I}} \in \bmod \left(\mathcal{K}^{\prime}\right)\right\}$. 
Proof of Proposition 4.4 We first show that $\operatorname{FM}\left(\mathcal{K} \circ_{P} \mathcal{K}^{\prime}\right)=\mathbb{F}$ where

$$
\mathbb{F}=\bigcup_{\Sigma \in \mathrm{MD}_{P}\left(\mathcal{K}, \mathcal{K}^{\prime}\right)}\left\{\mathcal{F}^{\prime} \in \operatorname{FM}\left(\mathcal{K}^{\prime}\right) \mid \mathcal{F} \sim \bar{\Sigma} \mathcal{F}^{\prime} \text { for some } \mathcal{F} \in \operatorname{FM}(\mathcal{K})\right\} .
$$

For each $\mathcal{F}^{\prime} \in \operatorname{FM}\left(\mathcal{K} \circ_{P} \mathcal{K}^{\prime}\right)$, by the definition of P-revision, there is some $\mathcal{F} \in \mathrm{FM}(\mathcal{K})$ such that $d_{P}\left(\mathcal{F}, \mathcal{F}^{\prime}\right) \cap \operatorname{MD}_{P}\left(\mathcal{K}, \mathcal{K}^{\prime}\right) \neq \emptyset$. Suppose $\Sigma \in d_{P}\left(\mathcal{F}, \mathcal{F}^{\prime}\right) \cap \operatorname{MD}_{P}\left(\mathcal{K}, \mathcal{K}^{\prime}\right)$. Then, by the definition of P-distance, $\mathcal{F} \sim \bar{\Sigma} \mathcal{F}^{\prime}$. Hence, $\mathcal{F}^{\prime} \in \mathbb{F}$. Conversely, for each $\mathcal{F}^{\prime} \in \mathbb{F}$, there is some $\Sigma \in \operatorname{MD}_{P}\left(\mathcal{K}, \mathcal{K}^{\prime}\right)$ and some $\mathcal{F} \in \operatorname{FM}(\mathcal{K})$ such that $\mathcal{F} \sim \bar{\Sigma}_{\bar{\Sigma}} \mathcal{F}^{\prime}$. By the definition of P-distance, $\Sigma \in d_{P}\left(\mathcal{F}, \mathcal{F}^{\prime}\right)$, and that is, $\Sigma \in d_{P}\left(\mathcal{F}, \mathcal{F}^{\prime}\right) \cap \operatorname{MD}_{P}\left(\mathcal{K}, \mathcal{K}^{\prime}\right)$. Then, by the definition of P-revision, $\mathcal{F}^{\prime} \in \mathrm{FM}\left(\mathcal{K} \circ_{P} \mathcal{K}^{\prime}\right)$. We have shown that $\operatorname{FM}\left(\mathcal{K} \circ_{P} \mathcal{K}^{\prime}\right)=\mathbb{F}$. Note that $\mathbb{F}$ is

$$
\bigcup_{\Sigma \in \operatorname{MD}_{P}\left(\mathcal{K}, \mathcal{K}^{\prime}\right)}\left(\operatorname{FM}\left(\mathcal{K}^{\prime}\right) \cap\left\{\mathcal{F}^{\prime} \mid \mathcal{F}^{\prime} \sim_{\Sigma} \mathcal{F} \text { for some } \mathcal{F} \in \operatorname{FM}(\mathcal{K})\right\}\right) .
$$

From Lemma B.2, that is,

$$
\bigcup_{\Sigma \in \mathrm{MD}_{P}\left(\mathcal{K}, \mathcal{K}^{\prime}\right)}\left(\operatorname{FM}\left(\mathcal{K}^{\prime}\right) \cap \mathrm{FM}\left(\text { forget }^{U}(\mathcal{K}, \Sigma)\right)\right) .
$$

From Proposition 3.18 we have shown that $\mathcal{K}{ }_{{ }_{P}} \mathcal{K}^{\prime}=\left\{\right.$ forget $\left.^{U}(\mathcal{K}, \Sigma) \cup \mathcal{K}^{\prime} \mid \Sigma \in \operatorname{MD}_{P}\left(\mathcal{K}, \mathcal{K}^{\prime}\right)\right\}$.

Proposition 4.7 For two KBs $\mathcal{K}$ and $\mathcal{K}^{\prime}, \operatorname{FM}\left(\mathcal{K} \circ_{P} \mathcal{K}^{\prime}\right)=\left\{\mathcal{F}_{\mathcal{I}} \mid \mathcal{I} \in \bmod \left(\mathcal{K} \circ_{P^{*}} \mathcal{K}^{\prime}\right)\right\}$

To prove Proposition 4.7, we observe that for two DL interpretations $\mathcal{I}_{1}$ and $\mathcal{I}_{2}$ with $d_{P^{*}}\left(\mathcal{I}_{1}, \mathcal{I}_{2}\right)=\Sigma$, suppose $\mathcal{I}_{1}$ and $\mathcal{I}_{2}$ induce $\mathcal{F}_{1}$ and $\mathcal{F}_{2}$, respectively, then $\mathcal{F}_{1} \sim_{\bar{\Sigma}} \mathcal{F}_{2}$. Moreover, we show the following two lemmas.

LEMMA B.3. Let $\Sigma \subseteq \Sigma_{\mathcal{S}}$, and $\mathcal{F}_{1}, \mathcal{F}_{2}$ be two featured interpretations s.t. $\mathcal{F}_{1} \sim_{\bar{\Sigma}} \mathcal{F}_{2}$. Then, there exist DL interpretations $\mathcal{I}_{1}$ and $\mathcal{I}_{2}$ inducing $\mathcal{F}_{1}$ and $\mathcal{F}_{2}$, respectively, s.t. $d_{P^{*}}\left(\mathcal{I}_{1}, \mathcal{I}_{2}\right)=\Sigma$.

Proof Let $\mathcal{F}_{i}=\left\langle\Xi_{i}, \mathcal{H}_{i}\right\rangle$ for $i=1,2$. By Proposition 3.13 there exists interpretations $\mathcal{I}_{1}$ and $\mathcal{I}_{0}$ inducing $\mathcal{F}_{1}$ and $\mathcal{F}_{2}$, respectively. From the construction in the proof of Proposition 3.13, we can find such interpretations with infinite domains $\Delta^{\mathcal{I}_{1}}$ and $\Delta^{\mathcal{I}_{0}}$, by making infinitely many copies of domain elements. As $\mathcal{F}_{1} \sim_{\bar{\Sigma}} \mathcal{F}_{2}$, for each $\tau_{1} \in \Xi_{1}$ there exists a $\tau_{2} \in \Xi_{2}$ such that $\tau_{1} \sim_{\bar{\Sigma}} \tau_{2}$, and vice versa. We can build a isomorphic mapping $\mu: \Delta^{\mathcal{I}_{1}} \rightarrow \Delta^{\mathcal{I}_{0}}$ between the domain elements of $\mathcal{I}_{1}$ and $\mathcal{I}_{0}$, such that $\tau^{\mathcal{I}_{1}}(d) \sim_{\bar{\Sigma}} \tau^{\mathcal{I}_{0}}(\mu(d))$ for each $d \in \Delta^{\mathcal{I}_{1}}$. Moreover, $\mu\left(a^{\mathcal{I}_{1}}\right)=a^{\mathcal{I}_{0}}$ for each individual $a \in \mathcal{S}_{I}$.

We will construct $\mathcal{I}_{2}$ from $\mathcal{I}_{1}$ and $\mathcal{I}_{0}$ as follows:

$-\Delta^{\mathcal{I}_{2}}=\Delta^{\mathcal{I}_{1}}$

- for each $a \in \mathcal{S}_{I}, a^{\mathcal{I}_{2}}=a^{\mathcal{I}_{1}}$;

- for each concept name $A \in \mathcal{S}_{C}$, if $A \in \Sigma, A^{\mathcal{I}_{2}}=\left\{d \in \Delta^{\mathcal{I}_{1}} \mid \mu(d) \in A^{\mathcal{I}_{0}}\right\}$; otherwise if $A \notin \Sigma$, $A^{\mathcal{I}_{2}}=A^{\mathcal{I}_{1}}$

- for each role name $P \in \mathcal{S}_{R}$, if $P \in \Sigma$, then $P^{\mathcal{I}_{2}}=\left\{(d, e) \in \Delta^{\mathcal{I}_{1}} \times \Delta^{\mathcal{I}_{1}} \mid(\mu(d), \mu(e)) \in P^{\mathcal{I}_{0}}\right\}$; otherwise if $P \notin \Sigma, P^{\mathcal{I}_{2}}=P^{\mathcal{I}_{1}}$.

By the construction of $\mathcal{I}_{2}$, it is clear that $d_{P^{*}}\left(\mathcal{I}_{1}, \mathcal{I}_{2}\right)=\Sigma$. We only need to show that $\mathcal{I}_{2}$ induces $\mathcal{F}_{2}=\left\langle\Xi_{2}, \mathcal{H}_{2}\right\rangle$.

For each concept name $A \in \mathcal{S}_{C}$ and each domain element $d \in \Delta^{\mathcal{I}_{1}}$, if $A \in \Sigma$, as $A^{\mathcal{I}_{2}}=\{d \in$ $\left.\Delta^{\mathcal{I}_{1}} \mid \mu(d) \in A^{\mathcal{I}_{0}}\right\}, d \in A^{\mathcal{I}_{2}}$ iff $\mu(d) \in A^{\mathcal{I}_{0}}$. If $A \in \mathcal{S}_{C} \backslash \Sigma$, as $A^{\mathcal{I}_{2}}=A^{\mathcal{I}_{1}}, d \in A^{\mathcal{I}_{2}}$ iff $d \in A^{\mathcal{I}_{1}}$. In this case, from $\tau^{\mathcal{I}_{1}}(d) \sim_{\bar{\Sigma}} \tau^{\mathcal{I}_{0}}(\mu(d)), d \in A^{\mathcal{I}_{1}}$ iff $\mu(d) \in A^{\mathcal{I}_{0}}$, and again, $d \in A^{\mathcal{I}_{2}}$ iff $\mu(d) \in A^{\mathcal{I}_{0}}$. Similarly, we can show that for any concept of the form $\geqslant n R$ over $\mathcal{S}, d \in(\geqslant n R)^{\mathcal{I}_{2}}$ iff $\mu(d) \in(\geqslant n R)^{\mathcal{I}_{0}}$. Thus, $d$ induces $\tau$ in $\mathcal{I}_{2}$ iff $\mu(d)$ induces $\tau$ in $\mathcal{I}_{0}$ for any $\mathcal{S}$-type $\tau$. We have shown that $\mathcal{I}_{2}$ induces exactly $\Xi_{2}$. 
For each $B(a) \in \mathcal{H}_{2}$ with $B$ a basic concept, as shown above, $a^{\mathcal{I}_{2}} \in B^{\mathcal{I}_{2}}$ iff $\mu\left(a^{\mathcal{I}_{2}}\right) \in B^{\mathcal{I}_{0}}$. Since $\mu\left(a^{\mathcal{I}_{2}}\right)=\mu\left(a^{\mathcal{I}_{1}}\right)=a^{\mathcal{I}_{0}}, a^{\mathcal{I}_{2}} \in B^{\mathcal{I}_{2}}$ iff $a^{\mathcal{I}_{0}} \in B^{\mathcal{I}_{0}}$. For each $P(a, b)$ or $P^{-}(b, a)$ in $\mathcal{H}_{2}$, if $P \in \Sigma$, as $P^{\mathcal{I}_{2}}=\left\{(d, e) \in \Delta^{\mathcal{I}_{1}} \times \Delta^{\mathcal{I}_{1}} \mid(\mu(d), \mu(e)) \in P^{\mathcal{I}_{0}}\right\},\left(a^{\mathcal{I}_{2}}, b^{\mathcal{I}_{2}}\right) \in P^{\mathcal{I}_{2}}$ iff $\left(\mu\left(a^{\mathcal{I}_{2}}\right), \mu\left(b^{\mathcal{I}_{2}}\right)\right) \in P^{\mathcal{I}_{0}}$. That is, $\left(a^{\mathcal{I}_{2}}, b^{\mathcal{I}_{2}}\right) \in P^{\mathcal{I}_{2}}$ iff $\left(a^{\mathcal{I}_{0}}, b^{\mathcal{I}_{0}}\right) \in P^{\mathcal{I}_{0}}$. If $P \in \mathcal{S}_{R} \backslash \Sigma, P^{\mathcal{I}_{2}}=P^{\mathcal{I}_{1}}$, and thus $\left(a^{\mathcal{I}_{2}}, b^{\mathcal{I}_{2}}\right) \in P^{\mathcal{I}_{2}}$ iff $\left(a^{\mathcal{I}_{1}}, b^{\mathcal{I}_{1}}\right) \in P^{\mathcal{I}_{1}}$. In this case, from $\mathcal{F}_{1} \sim_{\bar{\Sigma}} \mathcal{F}_{2}, \mathcal{I}_{1}$ satisfies $P(a, b)$ iff $\mathcal{I}_{0}$ satisfies $P(a, b)$. Hence, $\left(a^{\mathcal{I}_{1}}, b^{\mathcal{I}_{1}}\right) \in P^{\mathcal{I}_{1}}$ iff $\left(a^{\mathcal{I}_{0}}, b^{\mathcal{I}_{0}}\right) \in P^{\mathcal{I}_{0}}$. That is, $\left(a^{\mathcal{I}_{2}}, b^{\mathcal{I}_{2}}\right) \in P^{\mathcal{I}_{2}}$ iff $\left(a^{\mathcal{I}_{0}}, b^{\mathcal{I}_{0}}\right) \in P^{\mathcal{I}_{0}}$. We have shown that $\mathcal{I}_{2}$ induces exactly $\mathcal{H}_{2}$.

Lemma B.4. Let $\mathcal{K}, \mathcal{K}^{\prime}$ be two KBs and $\mathcal{S}=\operatorname{sig}\left(\mathcal{K} \cup \mathcal{K}^{\prime}\right)$. Then, $\operatorname{MD}_{P}\left(\mathcal{K}, \mathcal{K}^{\prime}\right)=$ $\operatorname{MD}_{P^{*}}\left(\mathcal{K}, \mathcal{K}^{\prime}\right)$.

Proof Let $\Sigma \subseteq \Sigma_{\mathcal{S}}$. Suppose $\Sigma \notin \mathrm{MD}_{P^{*}}\left(\mathcal{K}, \mathcal{K}^{\prime}\right)$, then there are two cases: either there exists no pair of models $\mathcal{I}$ and $\mathcal{I}^{\prime}$ of $\mathcal{K}$ and $\mathcal{K}^{\prime}$, respectively, such that $d_{P^{*}}\left(\mathcal{I}, \mathcal{I}^{\prime}\right)=\Sigma$; or $\Sigma$ is not minimal. In the first case, $\Sigma \notin \mathrm{MD}_{P}\left(\mathcal{K}, \mathcal{K}^{\prime}\right)$. Suppose otherwise, $\Sigma \in \mathrm{MD}_{P}\left(\mathcal{K}, \mathcal{K}^{\prime}\right)$ and by the definition of $\mathrm{P}$ distances, there are featured models $\mathcal{F}$ and $\mathcal{F}^{\prime}$ of respectively, $\mathcal{K}$ and $\mathcal{K}^{\prime}$, such that $\mathcal{F} \sim_{\bar{\Sigma}} \mathcal{F}^{\prime}$. From Lemma B.3 there exist models $\mathcal{I}$ and $\mathcal{I}^{\prime}$ inducing $\mathcal{F}$ and $\mathcal{F}^{\prime}$, respectively, such that $d_{P^{*}}\left(\mathcal{I}, \mathcal{I}^{\prime}\right)=\Sigma$. Moreover, from Proposition 3.17 $\mathcal{I}$ and $\mathcal{I}^{\prime}$ are models of $\mathcal{K}$ and $\mathcal{K}^{\prime}$, respectively. In the second case, there exist models $\mathcal{I}$ and $\mathcal{I}^{\prime}$ of $\mathcal{K}$ and $\mathcal{K}^{\prime}$, respectively, such that $d_{P^{*}}\left(\mathcal{I}, \mathcal{I}^{\prime}\right)=\Sigma^{\prime}$ for some $\Sigma^{\prime} \subset \Sigma$. Thus, for the two featured interpretations $\mathcal{F}$ and $\mathcal{F}^{\prime}$ induced by $\mathcal{I}$ and $\mathcal{I}^{\prime}$, respectively, $\mathcal{F} \sim_{\overline{\Sigma^{\prime}}} \mathcal{F}^{\prime}$. From Proposition 3.17, $\mathcal{F} \in \mathrm{FM}(\mathcal{K})$ and $\mathcal{F}^{\prime} \in \mathrm{FM}\left(\mathcal{K}^{\prime}\right)$. By the definitions of P-distances and $\operatorname{MD}_{P}\left(\mathcal{K}, \mathcal{K}^{\prime}\right), \Sigma \notin \operatorname{MD}_{P}\left(\mathcal{K}, \mathcal{K}^{\prime}\right)$.

Suppose $\Sigma \notin \operatorname{MD}_{P}\left(\mathcal{K}, \mathcal{K}^{\prime}\right)$, then there are also two cases: either there exists no pair of featured models $\mathcal{F}$ and $\mathcal{F}^{\prime}$ of $\mathcal{K}$ and $\mathcal{K}^{\prime}$, respectively, such that $\mathcal{F} \sim \bar{\Sigma} \mathcal{F}^{\prime}$; or $\Sigma$ is not minimal. In the first case, $\Sigma \notin \operatorname{MD}_{P^{*}}\left(\mathcal{K}, \mathcal{K}^{\prime}\right)$. Suppose otherwise, $\Sigma \in \operatorname{MD}_{P^{*}}\left(\mathcal{K}, \mathcal{K}^{\prime}\right)$ and there are models $\mathcal{I}$ and $\mathcal{I}^{\prime}$ of $\mathcal{K}$ and $\mathcal{K}^{\prime}$, respectively, such that $d_{P^{*}}\left(\mathcal{I}, \mathcal{I}^{\prime}\right)=\Sigma$. Then, for the two featured interpretations $\mathcal{F}$ and $\mathcal{F}^{\prime}$ induced by $\mathcal{I}$ and $\mathcal{I}^{\prime}$, respectively, $\mathcal{F} \sim_{\bar{\Sigma}} \mathcal{F}^{\prime}$. Moreover, from Proposition 3.17, $\mathcal{F} \in \operatorname{FM}(\mathcal{K})$ and $\mathcal{F}^{\prime} \in \operatorname{FM}\left(\mathcal{K}^{\prime}\right)$. In the second case, there exist featured models $\mathcal{F}$ and $\mathcal{F}^{\prime}$ of respectively, $\mathcal{K}$ and $\mathcal{K}^{\prime}$, such that $\mathcal{F} \sim \overline{\Sigma^{\prime}} \mathcal{F}^{\prime}$ and $\Sigma^{\prime} \subset \Sigma$. From Lemma $B$.3 there exist models $\mathcal{I}$ and $\mathcal{I}^{\prime}$ inducing $\mathcal{F}$ and $\mathcal{F}^{\prime}$, respectively, such that $d_{P^{*}}\left(\mathcal{I}, \mathcal{I}^{\prime}\right)=\Sigma^{\prime}$. From Proposition 3.17, $\mathcal{I}$ and $\mathcal{I}^{\prime}$ are models of $\mathcal{K}$ and $\mathcal{K}^{\prime}$, respectively. Thus, $\Sigma \notin \mathrm{MD}_{P^{*}}\left(\mathcal{K}, \mathcal{K}^{\prime}\right)$.

With Lemmas B.3 and B.4, we can show the proof for Proposition 4.7

Proof of Proposition 4.7 For the " $\supseteq$ " direction: for each $\mathcal{I} \in \bmod \left(\mathcal{K} \circ_{P^{*}} \mathcal{K}^{\prime}\right), \mathcal{I} \in \bmod \left(\mathcal{K}^{\prime}\right)$, and there exists $\mathcal{I}^{\prime} \in \bmod (\mathcal{K})$ with $d_{P^{*}}\left(\mathcal{I}, \mathcal{I}^{\prime}\right)=\Sigma$ for some $\Sigma \in \operatorname{MD}_{P^{*}}\left(\mathcal{K}, \mathcal{K}^{\prime}\right)$. By Lemma B.4. $\Sigma \in \operatorname{MD}_{P}\left(\mathcal{K}, \mathcal{K}^{\prime}\right)$. Let $\mathcal{F}^{\prime}$ be the featured interpretation induced by $\mathcal{I}^{\prime}$. Then, $\mathcal{F}_{\mathcal{I}} \sim_{\bar{\Sigma}} \mathcal{F}^{\prime}$. By Proposition 3.17, $\mathcal{F}^{\prime} \in \mathrm{FM}(\mathcal{K})$. In the proof of Proposition 4.4, we have shown that

$$
\operatorname{FM}\left(\mathcal{K} \circ_{P} \mathcal{K}^{\prime}\right)=\bigcup_{\Sigma \in \operatorname{MD}_{P}\left(\mathcal{K}, \mathcal{K}^{\prime}\right)}\left\{\mathcal{F}^{\prime} \in \operatorname{FM}\left(\mathcal{K}^{\prime}\right) \mid \mathcal{F} \sim_{\bar{\Sigma}} \mathcal{F}^{\prime} \text { for some } \mathcal{F} \in \operatorname{FM}(\mathcal{K})\right\}
$$

Hence, $\mathcal{F}_{\mathcal{I}} \in \operatorname{FM}\left(\mathcal{K} \circ_{P} \mathcal{K}^{\prime}\right)$.

For the " $\subseteq$ " direction: for each $\mathcal{F} \in \operatorname{FM}\left(\mathcal{K} \circ_{P} \mathcal{K}^{\prime}\right)$, by the above equation, $\mathcal{F} \in \operatorname{FM}\left(\mathcal{K}^{\prime}\right)$ and there exists $\overline{\mathcal{F}}^{\prime} \in \mathrm{FM}(\mathcal{K})$ such that $\mathcal{F} \sim_{\bar{\Sigma}} \mathcal{F}^{\prime}$ for some $\Sigma \in \operatorname{MD}_{P}\left(\mathcal{K}, \mathcal{K}^{\prime}\right)$. By Lemma B.4. $\Sigma \in$ $\operatorname{MD}_{P^{*}}\left(\mathcal{K}, \mathcal{K}^{\prime}\right)$. Note that we have shown in Lemma B.3 that there exist models $\mathcal{I}$ and $\mathcal{I}^{\prime}$ inducing $\mathcal{F}$ and $\mathcal{F}^{\prime}$, respectively, such that $d_{P^{*}}\left(\mathcal{I}, \mathcal{I}^{\prime}\right)=\Sigma$. Moreover, $\mathcal{I} \in \bmod \left(\mathcal{K}^{\prime}\right)$ and $\mathcal{I}^{\prime} \in \bmod (\mathcal{K})$. Thus, $\mathcal{I} \in \bmod \left(\mathcal{K} \circ_{P^{*}} \mathcal{K}^{\prime}\right)$ and $\mathcal{F} \in\left\{\mathcal{F}_{\mathcal{I}} \mid \mathcal{I} \in \bmod \left(\mathcal{K} \circ_{P^{*}} \mathcal{K}^{\prime}\right)\right\}$.

\section{PROOFS FOR SECTION 5}

Lemma 5.2 Let $\left|\mathcal{S}_{R}\right|=m$ and $\left|\mathcal{S}_{I}\right|=n$. Given a featured interpretation $\langle\Xi, \mathcal{H}\rangle$, a featured interpretation $\left\langle\Xi^{*}, \mathcal{H}\right\rangle$ exists such that (1) $\Xi^{*} \subseteq \Xi$, (2) $\left|\Xi^{*}\right| \leq 2 m+n$, and (3) $\left\langle\Xi^{*} \cup \Xi^{\prime}, \mathcal{H}\right\rangle$ is a featured interpretation for any type set $\Xi^{\prime} \subseteq \Xi$.

Proof From Definition 3.10 , for each role $P \in \mathcal{S}_{R}$, if $\exists P$ occurs in some type in $\Xi$, then $\exists P^{-}$ must occur in some type in $\Xi$. Let $\Xi_{R}$ be a minimal subset of $\Xi$ that contains at least one type 
containing $\exists P^{-}$for each $\exists P$ occurring in $\Xi$, and at least one type containing $\exists P$ for each $\exists P^{-}$in $\Xi$. Clearly, $\left|\Xi_{R}\right| \leq 2 m$.

Furthermore, $\Xi$ contains a type of $a$ in $\mathcal{H}$ for each individual $a$ occurring in $\mathcal{H}$. Let $\Xi_{I}$ be a minimal subset of $\Xi$ that contains at least one type of $a$ for each $a$ in $\mathcal{H}$. Let $\Xi^{*}=\Xi_{R} \cup \Xi_{I}$, and $\left|\Xi^{*}\right| \leq 2 m+n$. It is easy to verify from Definition 3.10 that $\left\langle\Xi^{*}, \mathcal{H}\right\rangle$ is a featured interpretation.

Lemma 5.3 Let $\left|\mathcal{S}_{R}\right|=m,\left|\mathcal{S}_{I}\right|=n$, and $\mathcal{F}_{i}=\left\langle\Xi_{i}, \mathcal{H}_{i}\right\rangle(i=1,2)$ be a pair of featured interpretations. Suppose $\Sigma \in d_{P}\left(\mathcal{F}_{1}, \mathcal{F}_{2}\right)$, then a pair of featured interpretations $\mathcal{F}_{i}^{P}=\left\langle\Xi_{i}^{P}, \mathcal{H}_{i}\right\rangle(i=1,2)$ exist, such that for $i=1,2$, (1) $\Xi_{i}^{P} \subseteq \Xi_{i}$, (2) $\left|\Xi_{i}^{P}\right| \leq 4 m+2 n$, and (3) $\Sigma \in d_{P}\left(\mathcal{F}_{1}^{P}, \mathcal{F}_{2}^{P}\right)$.

There also exist a pair of featured interpretations $\mathcal{F}_{i}^{S}=\left\langle\Xi_{i}^{S}, \mathcal{H}_{i}\right\rangle(i=1,2)$, such that for $i=1,2$, (1') $\Xi_{i}^{S} \subseteq \Xi_{i}$, (2') $\left|\Xi_{i}^{S}\right| \leq 4 m+2 n$, and (3') $\Xi_{1}^{S} \triangle \Xi_{2}^{S} \subseteq \Xi_{1} \triangle \Xi_{2}$.

Proof For the first half of the lemma, to obtain $\Xi_{i}^{P}(i=1,2)$, we first construct $\Xi_{i}^{*}(i=1,2)$ as in the proof of Lemma 5.2. Then, each $\left\langle\Xi_{i}^{*}, \mathcal{H}_{i}\right\rangle$ is a featured interpretation and $\left|\Xi_{i}^{*}\right| \leq 2 m+n$. Since $\Sigma \in d_{P}\left(\mathcal{F}_{1}, \mathcal{F}_{2}\right)$, by the definition of P-distance, we have $\mathcal{F}_{1} \sim \bar{\Sigma} \mathcal{F}_{2}$. That is, for each type $\tau_{2} \in \Xi_{2}$, there is a type $\tau_{1} \in \Xi_{1}$ such that $\tau_{1} \sim \bar{\Sigma} \tau_{2}$. Since $\Xi_{2}^{*} \subseteq \Xi_{2}$, there is such a $\tau_{1}$ for each $\tau_{2} \in \Xi_{2}^{*}$. Let $\tilde{\Xi}_{1}$ be a minimal subset of $\Xi_{1}$ containing such a $\tau_{1}$ for each $\tau_{2} \in \Xi_{2}^{*}$. Hence, $\left|\tilde{\Xi}_{1}\right| \leq 2 m+n$. Construct $\Xi_{1}^{P}=\Xi_{1}^{*} \cup \tilde{\Xi}_{1}$, and it satisfies (1) and (2). Let $\Xi_{2}^{P}$ be constructed in the same way, and it also satisfies (1) and (2). Let $\mathcal{F}_{i}^{P}=\left\langle\Xi_{i}^{P}, \mathcal{H}_{i}\right\rangle$ for $i=1,2$. From Lemma 5.2, both $\mathcal{F}_{i}^{P}$ 's are featured interpretations. Also, it is easy to verify that $\mathcal{F}_{1}^{P} \sim \bar{\Sigma}_{\Sigma} \mathcal{F}_{2}^{P}$. That is, $\Sigma \in d_{P}\left(\mathcal{F}_{1}^{P}, \mathcal{F}_{2}^{P}\right)$. Hence, $\mathcal{F}_{1}^{P}$ and $\mathcal{F}_{2}^{P}$ satisfy (3).

For the second half of the lemma, suppose $\Xi_{i}^{*}(i=1,2)$ is obtained as above. Let $\Xi_{1}^{S}=\Xi_{1}^{*} \cup\left(\Xi_{1} \cap\right.$ $\left.\Xi_{2}^{*}\right)$, and in the same way for $\Xi_{2}^{S}$. Then, $\Xi_{1}^{S} \subseteq \Xi_{1}$, and since $\left|\Xi_{1} \cap \Xi_{2}^{*}\right| \leq 2 m+n,\left|\Xi_{1}^{S}\right| \leq 4 m+2 n$. Similar conditions hold for $\Xi_{2}^{S}$, and hence $\Xi_{1}^{\bar{S}}$ and $\Xi_{2}^{S}$ both satisfy (1') and (2'). Also, it is clear that $\Xi_{1}^{S} \triangle \Xi_{2}^{S} \subseteq \Xi_{1} \triangle \Xi_{2}$, as $\Xi_{1}^{S} \backslash \Xi_{2}^{S} \subseteq \Xi_{1} \backslash \Xi_{2}$ and its symmetric. Let $\mathcal{F}_{i}^{S}=\left\langle\Xi_{i}^{S}, \mathcal{H}_{i}\right\rangle$ for $i=1,2$, and they are both featured interpretations and satisfy (3').

Note that in the above proof, for an arbitrary type $\tau \in \Xi_{1}$, suppose $\tau$ is added to $\Xi_{1}^{*}$ (and thus $\left|\Xi_{1}^{*}\right| \leq 2 m+n+1$, we can still construct $\Xi_{1}^{P}$ and $\Xi_{1}^{S}$ in the same manner, such that $\left|\Xi_{1}^{P}\right| \leq$ $4 m+2 n+1$ and $\left|\Xi_{1}^{S}\right| \leq 4 m+2 n+1$.

Lemma 5.4 Let $\mathcal{K}$ and $\overline{\mathcal{K}}^{\prime}$ be two KBs and $\alpha$ an axiom. For $X=P$ or $S$, if a featured model $\mathcal{F} \in$ $\operatorname{FM}\left(\mathcal{K}^{\prime}\right)$ is $X$-closest to $\mathcal{K}$ and does not satisfy $\alpha$, then there exists a featured model $\mathcal{F}^{\prime} \in \operatorname{FM}\left(\mathcal{K}^{\prime}\right)$ of polynomial size that is $X$-closest to $\mathcal{K}$ and does not satisfy $\alpha$.

Proof As $\mathcal{F}$ is $\mathrm{P}$-closest to $\mathcal{K}$, there exists a featured model $\mathcal{F}_{1}$ of $\mathcal{K}$ such that the $\mathrm{P}$-distance between $\mathcal{F}$ and $\mathcal{F}_{1}$ is minimal. That is, there exists some $\Sigma \in d_{P}\left(\mathcal{F}_{1}, \mathcal{F}\right) \cap \operatorname{MD}_{P}\left(\mathcal{K}, \mathcal{K}^{\prime}\right)$. From Lemma 5.3 we can construct $\mathcal{F}^{P}$ and $\mathcal{F}_{1}^{P}$ such that $\Sigma \in d_{P}\left(\mathcal{F}_{1}^{P}, \mathcal{F}^{P}\right)$. That is, $d_{P}\left(\mathcal{F}_{1}^{P}, \mathcal{F}^{P}\right) \cap$ $\operatorname{MD}_{P}\left(\mathcal{K}, \mathcal{K}^{\prime}\right) \neq \emptyset$. To conclude that $\mathcal{F}^{P}$ is P-closest to $\mathcal{K}$, we only need to show that $\mathcal{F}_{1}^{P}$ is a featured model of $\mathcal{K}$. Yet we first show that $\mathcal{F}^{P}$ is a featured model of $\mathcal{K}^{\prime}$, and $\mathcal{F}_{1}^{P}$ being a featured model of $\mathcal{K}$ can be shown similarly.

To see that $\mathcal{F}^{P}$ is a featured model of $\mathcal{K}^{\prime}$, since $\Xi^{P} \subseteq \Xi$, for each concept inclusion $C \sqsubseteq D$ in $\mathcal{K}^{\prime}, \Xi$ satisfying $C \sqsubseteq D$ implies that $\Xi^{P}$ satisfies $C \sqsubseteq \bar{D}$. That is, $\mathcal{F}$ satisfying $C \sqsubseteq D$ implies that $\mathcal{F}^{P}$ satisfies $C \sqsubseteq D$. Also, as $\mathcal{F}^{P}$ has the same Herbrand set as $\mathcal{F}$, for each membership assertion $\beta$ in $\mathcal{K}^{\prime}, \mathcal{F}$ satisfying $\beta$ implies that $\mathcal{F}^{P}$ satisfies $\beta$. Hence, $\mathcal{F} \in \operatorname{FM}\left(\mathcal{K}^{\prime}\right)$ implies that $\mathcal{F}$ satisfies each concept inclusion and membership assertion in $\mathcal{K}^{\prime}$, which implies that $\mathcal{F}^{P}$ satisfies each concept inclusion and membership assertion in $\mathcal{K}^{\prime}$, and hence $\mathcal{F}^{P}$ is a featured model of $\mathcal{K}^{\prime}$. Similarly, $\mathcal{F}_{1}^{P}$ is a featured model of $\mathcal{K}$. Hence, $\mathcal{F}^{P}$ is P-closest to $\mathcal{K}$.

To see that $\mathcal{F}^{P}$ does not satisfy $\alpha$, suppose $\alpha$ is a concept inclusion, then by the definition of $\mathcal{F}$ (not) satisfying $\alpha$, there is a type $\tau$ in $\mathcal{F}$ that does not satisfy $\alpha$. By the discussion after the proof of Lemma 5.3. we can assume without loss of generality that $\tau$ is added into $\mathcal{F}^{P}$. Then, as $\mathcal{F}^{P}$ contains $\tau$, and $\mathcal{F}^{P}$ does not satisfy $\alpha$. Similarly, if $\alpha$ is a membership assertion, then $\mathcal{F}^{P}$ does not satisfy $\alpha$ as $\mathcal{F}^{P}$ has exactly the same Herbrand set as $\mathcal{F}$. 
In the same way, from $\mathcal{F}$ and a featured model $\mathcal{F}_{2}$ of $\mathcal{K}$ such that the S-distance between $\mathcal{F}$ and $\mathcal{F}_{2}$ is both F-minimal and H-minimal, we can construct $\mathcal{F}^{S}$ and $\mathcal{F}_{2}^{S}$ that are featured models of $\mathcal{K}^{\prime}$ and $\mathcal{K}$, respectively, and $\mathcal{F}^{S}$ does not satisfy $\alpha$. To see that $\mathcal{F}^{S}$ is S-closest to $\mathcal{K}$, by Lemma 5.3, $\mathcal{F}^{S}$ $\left(\mathcal{F}_{2}^{S}\right)$ has the same Herbrand set as $\mathcal{F}\left(\mathcal{F}_{2}\right)$, and also, $\Xi^{S} \triangle \Xi_{2}^{S} \subseteq \Xi \triangle \Xi_{2}$. Hence, $d_{F}\left(\mathcal{F}^{S}, \mathcal{F}_{2}^{S}\right) \subseteq$ $d_{F}\left(\mathcal{F}, \mathcal{F}_{2}\right)$. We have shown that $\mathcal{F}^{S}$ is S-closest to $\mathcal{K}$.

Proposition 5.5 Given a $K B \mathcal{K}$ and a signature $\mathcal{S}$, let $\left|\mathcal{S}_{R}\right|=m$ and $\left|\mathcal{S}_{I}\right|=n$. Suppose Algorithm 1 returns $\operatorname{Rep}(\mathcal{K})$, then the following conditions hold:

(1) $\operatorname{Rep}(\mathcal{K}) \subseteq \operatorname{FM}(\mathcal{K})$ and each featured model in $\operatorname{Rep}(\mathcal{K})$ has at most $4 m+2 n+2$ types.

(2) For another $K B \mathcal{K}^{\prime}$ on $\mathcal{S}$ and $X=P$ or $S$, Select $\left(\mathcal{K}, \mathcal{K}^{\prime}\right) \subseteq \operatorname{FM}\left(\mathcal{K} \circ_{X} \mathcal{K}^{\prime}\right)$; and for each $\mathcal{F} \in \operatorname{FM}\left(\mathcal{K} \circ_{X} \mathcal{K}^{\prime}\right)$ and a type $\tau$ in $\mathcal{F}$, there exists $\mathcal{F}^{\prime} \in \operatorname{Select}_{X}\left(\mathcal{K}, \mathcal{K}^{\prime}\right)$ that has the same Herbrand set as $\mathcal{F}$ and contains $\tau$.

Proof Condition (1) is clear from the discussions under Algorithm 1 . For Condition (2), consider a featured model $\mathcal{F}=\langle\Xi, \mathcal{H}\rangle \in \operatorname{FM}\left(\mathcal{K} \circ_{X} \mathcal{K}^{\prime}\right)$ and a featured model $\mathcal{F}_{1}=\left\langle\Xi_{1}, \mathcal{H}_{1}\right\rangle$ of $\operatorname{FM}(\mathcal{K})$ such that the X-distance between $\mathcal{F}$ and $\mathcal{F}_{1}$ is minimal. From Lemma 5.3, we first show that $\mathcal{F}^{X}=\left\langle\Xi^{X}, \mathcal{H}\right\rangle$ is in $\operatorname{Rep}\left(\mathcal{K}^{\prime}\right)$ and $\mathcal{F}_{1}^{X}=\left\langle\Xi_{1}^{X}, \mathcal{H}_{1}\right\rangle$ is in $\operatorname{Rep}(\mathcal{K})$, that is they can be generated by Algorithm 1 .

For $X=P$, we have $\mathcal{F} \sim \bar{\Sigma}_{\bar{\Sigma}} \mathcal{F}_{1}$ for some $\Sigma \in \operatorname{MD}_{P}\left(\mathcal{K}, \mathcal{K}^{\prime}\right)$. First, let $\Xi^{*}$ consist the type $\tau^{\mathcal{H}}(a)$ for each individual $a \in \mathcal{S}_{I}$ and a type from $\Xi$ containing $\exists R^{-}$for each type in $\Xi^{P}$ containing $\exists R$. Such a $\Xi^{*}$ can be generated by Line 7 and Lines 9-12 in Algorithm 1 Assume $\Xi_{1}^{*}$ is also generated in the same way (for $\Xi_{1}$ ). Finally, let $\tilde{\Xi}$ be a minimal subset of $\Xi$ containing a type $\tau_{1}^{\prime}$ with $\tau_{1} \sim_{\bar{\Sigma}} \tau_{1}^{\prime}$ for each type $\tau_{1}$ in $\Xi_{1}^{*}$. All the types in $\tilde{\Xi}$ is added to $\Xi^{*}$ by Line 8 . That is, $\Xi^{P}=\Xi^{*} \cup \tilde{\Xi}$ can be generated by Algorithm 1 . Hence, $\mathcal{F}^{P}=\left\langle\Xi^{P}, \mathcal{H}\right\rangle$ and $\mathcal{F}_{1}^{P}=\left\langle\Xi_{1}^{P}, \mathcal{H}_{1}\right\rangle$ can be generated by Algorithm 11. Also, $\mathcal{F}^{P} \sim_{\bar{\Sigma}} \mathcal{F}_{1}^{P}$. For $X=S, \Xi^{*}$ and $\Xi_{1}^{*}$ are generated as above. Then, all the types in $\Xi \cap \Xi_{1}^{*}$ are added to $\Xi^{*}$ by Line 8 . That is, $\Xi^{S}=\Xi^{*} \cup\left(\Xi \cap \Xi_{1}^{*}\right)$ can be generated by Algorithm 1 . Hence, $\mathcal{F}^{S}=\left\langle\Xi^{S}, \mathcal{H}\right\rangle$ and $\mathcal{F}_{1}^{S}=\left\langle\Xi_{1}^{S}, \mathcal{H}_{1}\right\rangle$ can be generated by Algorithm 1 . Also, their $S$ distance is the same as that between $\mathcal{F}$ and $\mathcal{F}_{1}$. We have shown that $\mathcal{F}^{X}=\left\langle\Xi^{X}, \mathcal{H}\right\rangle$ is in $\operatorname{Rep}\left(\mathcal{K}^{\prime}\right)$ and $\mathcal{F}_{1}^{X}=\left\langle\Xi_{1}^{X}, \mathcal{H}_{1}\right\rangle$ is in $\operatorname{Rep}(\mathcal{K})$. Moreover, it is easy to verify that $\mathcal{F}^{X} \in \operatorname{Select}_{X}\left(\mathcal{K}, \mathcal{K}^{\prime}\right)$, as the $\mathrm{X}$-distance between $\mathcal{F}^{X}$ and $\mathcal{F}_{1}^{X}$ is minimal.

Now, to show $\operatorname{Select}_{X}\left(\mathcal{K}, \mathcal{K}^{\prime}\right) \subseteq \operatorname{FM}\left(\mathcal{K} \circ_{X} \mathcal{K}^{\prime}\right)$, suppose by contradiction there is some $\mathcal{F}^{\prime} \in$ $\operatorname{Select}_{X}\left(\mathcal{K}, \mathcal{K}^{\prime}\right)$ and $\mathcal{F}^{\prime} \notin \operatorname{FM}\left(\mathcal{K} \circ_{X} \mathcal{K}^{\prime}\right)$. Note that $\mathcal{F}^{\prime} \in \operatorname{Rep}\left(\mathcal{K}^{\prime}\right) \subseteq \operatorname{FM}\left(\mathcal{K}^{\prime}\right)$. As $\mathcal{F}^{\prime} \notin \operatorname{FM}\left(\mathcal{K} \circ_{X}\right.$ $\left.\mathcal{K}^{\prime}\right)$, for each $\mathcal{F}_{1}^{\prime} \in \mathrm{FM}(\mathcal{K})$, there are $\mathcal{F} \in \mathrm{FM}\left(\mathcal{K}^{\prime}\right)$ and $\mathcal{F}_{1} \in \mathrm{FM}(\mathcal{K})$ that witness the nonminimality of the X-distance between $\mathcal{F}^{\prime}$ and $\mathcal{F}_{1}^{\prime}$. From the above discussions, we have $\mathcal{F}^{X} \in$ $\operatorname{Rep}\left(\mathcal{K}^{\prime}\right)$ and $\mathcal{F}_{1}^{X} \in \operatorname{Rep}(\mathcal{K})$ that witness the non-minimality of the $\mathrm{X}$-distance between $\mathcal{F}^{\prime}$ and $\mathcal{F}_{1}^{\prime}$. As $\mathcal{F}_{1}^{\prime}$ ranges over $\operatorname{FM}(\mathcal{K})$ and hence ranges over $\operatorname{Rep}(\mathcal{K})$. This contradicts the assumption that $\mathcal{F}^{\prime} \in \operatorname{Select}_{X}\left(\mathcal{K}, \mathcal{K}^{\prime}\right)$. We have shown that $\operatorname{Select}_{X}\left(\mathcal{K}, \mathcal{K}^{\prime}\right) \subseteq \operatorname{FM}\left(\mathcal{K} \circ_{X} \mathcal{K}^{\prime}\right)$.

For the second half of Condition (2), simply let $\mathcal{F}^{\prime}=\mathcal{F}^{X}$, and from the discussion after the proof of Lemma 5.3, we can assume without loss of generality that the type $\tau$ is added to $\Xi^{X}$ by Line 8 of Algorithm 1 